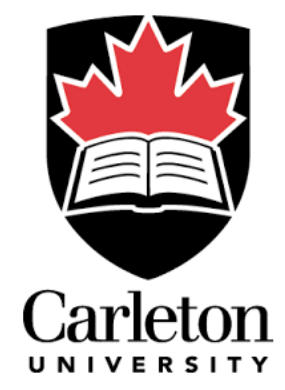

\title{
Functional Genomics in Yeast
}

\author{
By Maryam Hajikarimlou
}

A thesis submitted to the Faculty of Graduate and Postdoctoral

Affairs in partial fulfillment of the requirements for the degree of

\author{
Doctor of Philosophy \\ in \\ Department of Biology, \\ Ottawa-Carleton Institute of Biology
}

\author{
Carleton University \\ Ottawa, Ontario
}

(C)2020, Maryam Hajikarimlou 


\section{Abstract}

Living cells are complex biological systems with large networks of interactions between different macromolecules. A focus of systems biology is to study the dynamics of the living cells and their interactions through functional genomic approaches. High-throughput techniques and whole-genome screening experiments allow us to collect "big data" for various biochemical networks within the cell. The baker's yeast, Saccharomyces cerevisiae, is a single-cell eukaryotic model organism used for functional genomics approaches, the genome of which has considerable homology with the human genome. In this thesis, we used functional genomic approaches to study novel function(s) for genes and pathways affecting translation. The process of translation is an essential pathway that leads to the production of functional proteins for living cells. Dysregulation of this process and its associated pathways have been linked to many diseases, emphasizing the importance and need to investigate details of genes that influence this pathway. Much has been learned so far but there are still unknown regulations that require more examination.

One of the main objectives of this thesis is to discover and study novel function of genes that affect the translation of structured mRNAs in yeast. We designed different constructs to evaluate the effect of nearly 5000 non-essential genes on the translation of reporter genes. Through whole-genome screening experiments and follow up assays we proposed the heretofore unknown involvement of five genes in the translation pathway. In the current thesis, YTA6, YPRO96C, NAM7, PUS2, and RPL27B are proposed to be 
important for the translation of mRNAs with structured regions within their $5^{\prime}$-UTRs in yeast. In another study, we used translation in mitochondria as a strategy to investigate the ability of a specific $3^{\prime}$-UTR sequence to direct a reporter mRNA into yeast mitochondria. Lastly, using additional screenings we propose the involvement of dozens of more genes that seem to be important for the translation of structured mRNAs.

Together, our findings contribute to a better understanding of the translation of structured mRNAs. They also indicate that our overall understanding of the regulations of translation may require additional studies in years to come. 


\section{Acknowledgements}

I could not start without thanking my supervisor Ashkan for everything he has done for me and giving me the opportunity to work in his lab. You have always been more than a professor to me, and you have always taken the time to teach more than just biology. You have always taught about how to live life and be a good person by being kind, considerate and patient with your students. Your door is always open, you are always available to talk about anything, and have always given wonderful guidance about everything.

I would like to express my deepest appreciation to my committee Dr. Myron Smith and Dr. Martin Holcik for their valuable suggestions and support on my thesis. Dr. Kyle Biggar for all the collaborations we did and being friendly and giving practical suggestions all the way. I'm also deeply indebted to Dr. Owen Rowland for trusting in my abilities in teaching and guiding my teaching philosophy.

I would like to extend my sincere thanks to Suzanne Paterson and lan Pulsifer, for understanding and assistance in all the work involved with teaching as a TA. Adapting to a new teaching environment was a difficult transition, and you made the process seamless and helped me adapt to my roles and responsibilities effortlessly. Also I want to appreciate all the help and love from SarahAnn and loving memory of Laura who made everything in the department go smoother and easier. 
To my friends Katyoun, Barham, and Moshen your support, friendship and help with moving to Canada and getting settled in a new country when my family was thousands of miles away, made the difficult transition easier. Let alone all your help in the lab through the years. I couldn't have done it without you.

Thanks to all the people in the lab and office that made it a professional, collaborative and friendly environment to work in. Dan, Duale, Sarah, Narges, Natalia, Ryan, Taylor, Anna, Hemantha, Urvi, Tom, Alex, Michael, Sasi, Hayuin, Tim, Mat, Rowida, and Shannon I appreciate all your friendship and kindness.

To my loving and supporting family, everything I have achieved today started with you. From the love and support of my parents growing up, to my siblings, Farzin, Shirin, Fatemeh, Mehdi and Sepideh who pushed me to follow my dreams, I miss you all so very much and love you more than you can ever know. You all encouraged me to improve and become a better version of myself in different aspects of life. I am grateful to have the love of my nieces and nephew Rira, Bardia, Kimia, and Kiana, thinking about you always makes my heart melt and gives meaning to my life.

My sister Shirin, you believed in me no matter what. You always truly supported and believed that I was capable of great things, even when I didn't believe it myself. I am sure whatever I am achieving right now is just because you were there for me and cared for me no matter what.

Special thanks to Sarah, you questioned everything in the lab and made me think and understand science from different aspects, not to forget the motivation in health and 
fitness. Reza, Hanie, Shaghayegh and Mehdi you have the kindest heart and are one of the nicest people that I know, thanks for being there for me. I also want to thank Mark who has been a good friend specially through the hard times of writing my thesis and supporting me in this journey. Special thanks to my old friend Atena, I am lucky to have you as my friend you have always supported me in studying and believing in myself for the longest time, you taught me how to be a good student and I am always grateful for that.

My dearest friend Shirin, you always listened to whatever I was complaining about with an open mind and gave your honest opinion, whether I wanted to hear it or not. I could not get by without your daily emotional support and daily phone calls. Thanks for understanding, listening and helping me go over the hardest times. 


\section{Statement of contribution}

Chapter 2: Sensitivity of yeast to lithium chloride connects the activity of YTA6 and YPR096C to translation of structured mRNAs.

"Sensitivity of yeast to Lithium Chloride connects the activity of YTA6 and YPRO96C to translation of structured mRNAs", is the result of experiments primarily designed and carried out by myself. A group of undergraduate and graduate students assisted me and worked under my supervision. Narges Zare, Sarah Takallou, Tom Kazmirchuk, Nathalie Puchacz, and Sasi Jagadeeson helped me with large scale and small scale 6-galactosidase experiments and screenings. Houman Moteshareie, Mohsen Hooshyar, Katayoun Omidi, and Daniel Burnside helped with SGA and PSA analysis. I wrote the manuscript.

\section{Chapter 3: Lithium chloride toxicity is connected to the regulation of gene expression} in yeast.

"Lithium chloride toxicity is connected to the regulation of gene expression in yeast", is the result of experiments primarily designed and carried out by myself. A group of undergraduate and graduate students including Kathryn Hunt, Grace Kirby, and Sasi Jagadeeson helped me with large scale and small scale 6 -galactosidase experiments and screenings. Sarah Takallou and Katayoun Omidi helped me with SGA and PSA experiments. I wrote the first draft of the manuscript. 


\section{Chapter 4: A correlation between 3'-UTR of OXA1 gene and yeast mitochondrial translation.}

"A correlation between 3'-UTR of OXA1 gene and yeast mitochondrial translation", is the result of experiments primarily designed by myself and Noor Sunba. A group of undergraduate and graduate students helped me with b-galactosidase experiments including Sarah Takallou, Nathalia Puchacz, Houman Moteshareie, and Sasi Jagadeeson. Nazila Nazemof contributed to running the qRT-PCR experiments. Drug treatment optimization was performed by me with the help of Katayoun Omidi and Daniel Burnside. Mohsen Hooshyar contributed to analyzing the results and writing the manuscript. The manuscript was written by me.

\section{Chapter 5: Translation regulation of highly structured 5'-UTR constructs.}

"Translation regulation of highly structured 5'-UTR constructs", is the result of experiments primarily designed and carried out by me. Sarah Takallou and Sasi Jagadeeson helped me with B-galactosidase experiments and screenings. I wrote the initial manuscript for this project. 


\section{Table of contents}

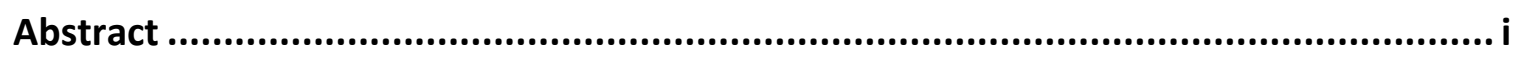

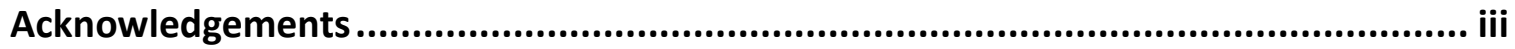

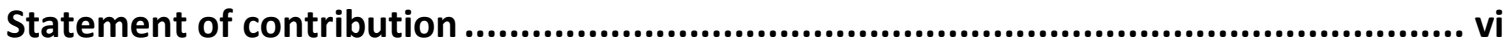

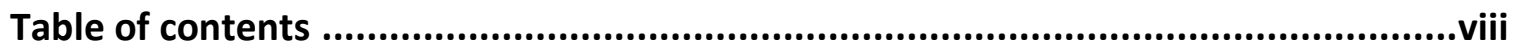

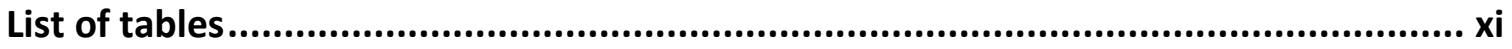

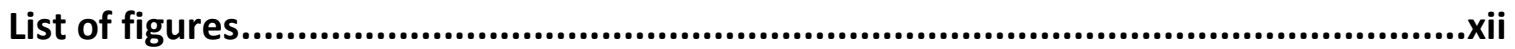

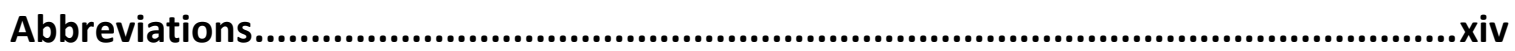

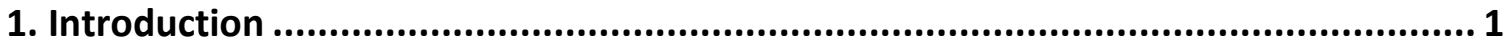

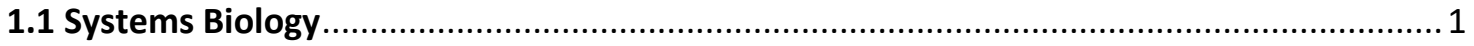

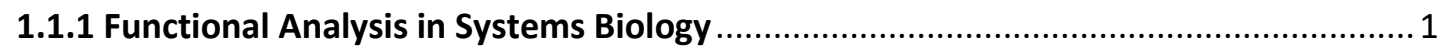

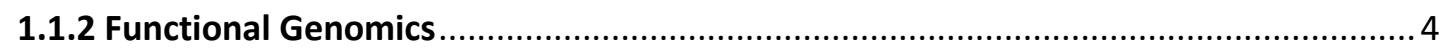

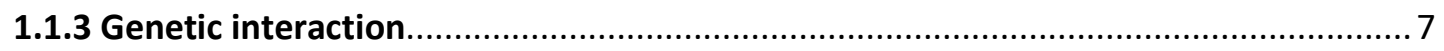

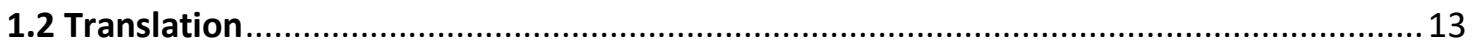

1.2.1 Translation pathway and the significance of studying it. ........................................ 13

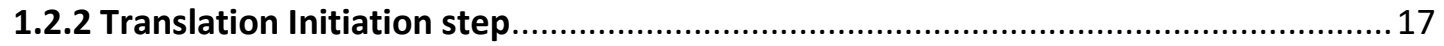

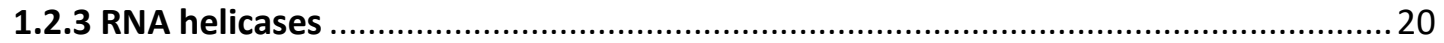

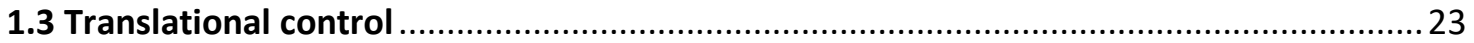

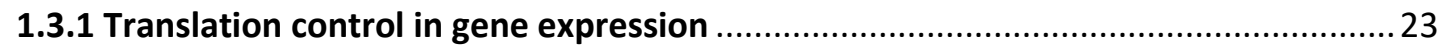

1.3.2 Translation control of mRNAs with structured 5'-UTRs ...........................................28

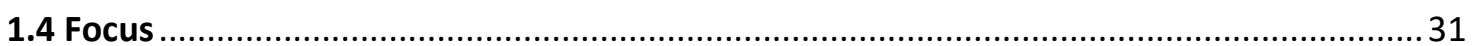

2. Sensitivity of yeast to lithium chloride connects the activity of YTA6 and YPR096C to translation of structured mRNAs .......................................................................... 34

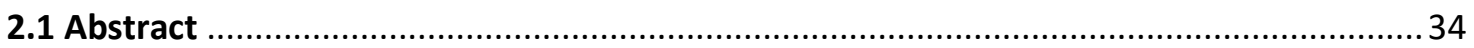

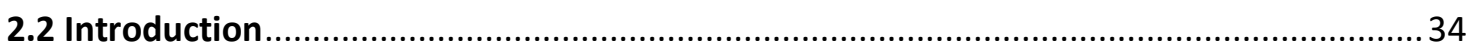

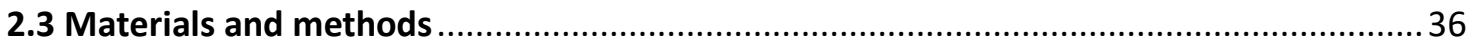

2.3.1 Strains, plasmids, gene collections and cell and DNA manipulations ........................36

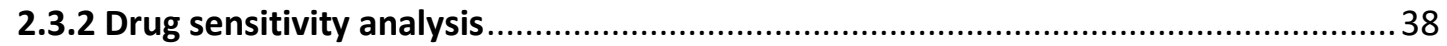

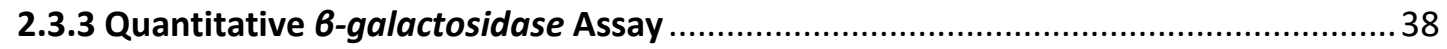

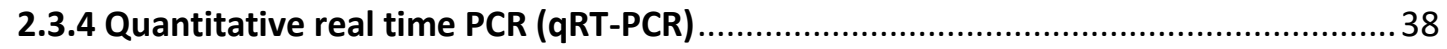

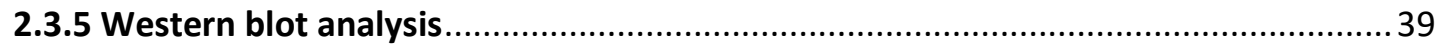




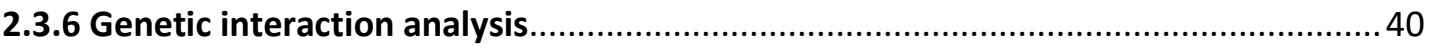

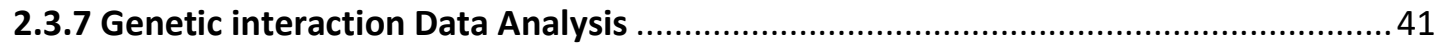

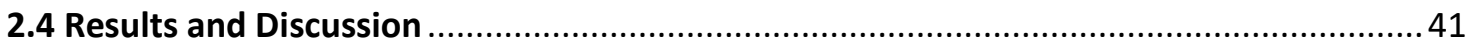

2.4.1 Deletion of YTA6 and YPR096C increases yeast sensitivity to Lithium ..................... 41

2.4.2 YTA6 and YPRO96C regulate the expression of PGM2 at the level of translation. .... 45

2.4.3 Translation of 6-galactosidase reporter mRNA with a hairpin structure is altered by YTA6 and YPRO96C

2.4.4 Genetic interaction analysis further connects the activity of YTA6 and YPR096C to protein biosynthesis

3. Lithium chloride toxicity is connected to the regulation of gene expression in yeast.

3.1 Abstract 56

3.2 Introduction .56

3.3 Materials and Methods .56

3.3.1 Strains, plasmids, gene collections and cell and DNA manipulations .59

3.3.2 Drug sensitivity analyses. .59

3.3.3 Quantitative 6-galactosidase Assay 61

3.3.4 Quantitative real time PCR (qRT-PCR) 61

3.3.5 Western blot analysis. 62

3.3.6 Genetic interaction analysis. 62

3.3.7 Genetic interaction Data Analysis 63

3.4 Results .64

3.4.1 Deletion of NAM7, PUS2 and RPL27B increases yeast sensitivity to Lithium Chloride.

3.4.2 NAM7, PUS2, and RPL27B regulate the expression of PGM2 at the level of translation.

3.4.3 Translation of 6 -galactosidase reporter mRNA with a hairpin structure is altered by NAM7, PUS2, and RPL27B.

3.4.4 Genetic interaction analysis further connects the activity of NAM7, PUS2, and $R P L 27 B$ to protein biosynthesis.

4. A correlation between $3^{\prime}$-UTR of OXA1 gene and yeast mitochondrial translation. 79

4.1 Abstract 79

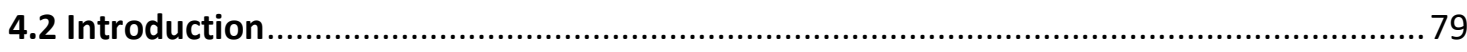

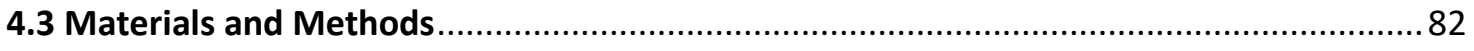

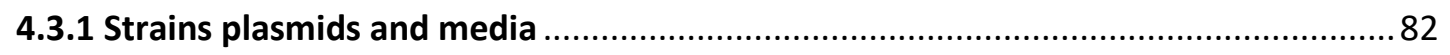




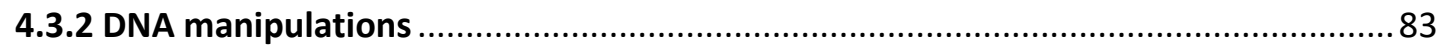

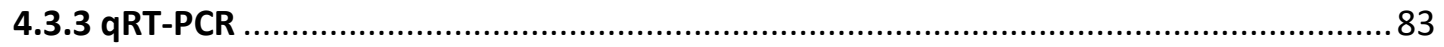

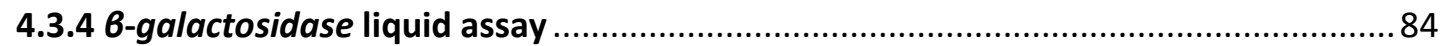

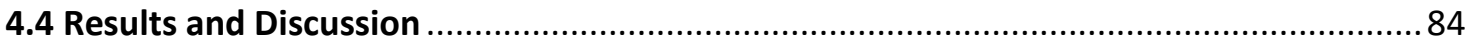

4.4.1 In isolated mitochondria, the activity and content of a B-galactosidase mRNA is

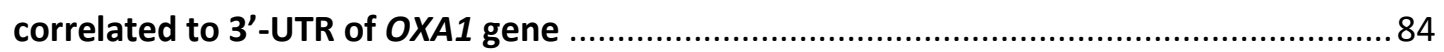

4.4.2 Observed 6-galactosidase activity contains signatures of mitochondrial translation.

5. Translation regulation of highly structured 5'-UTR constructs........................... 93

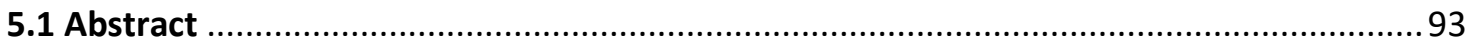

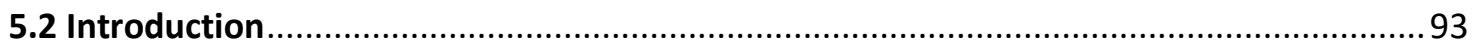

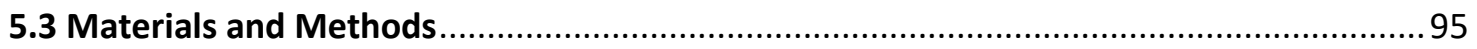

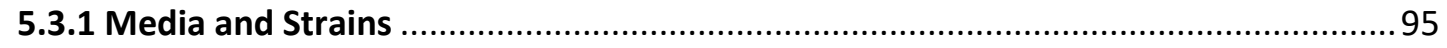

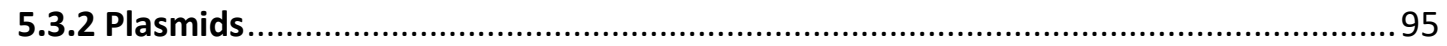

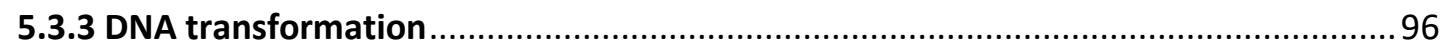

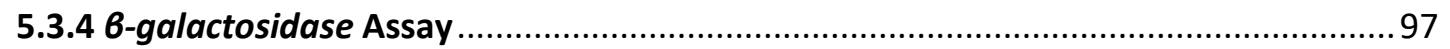

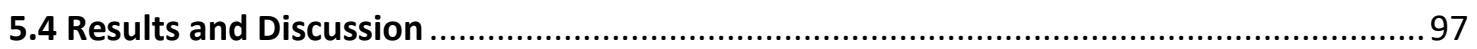

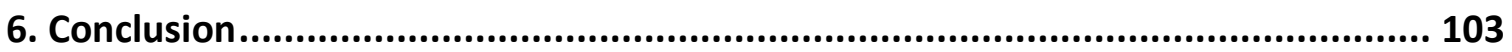

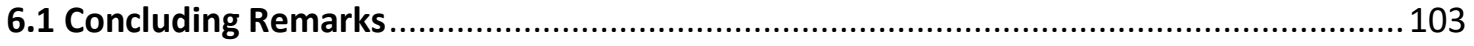

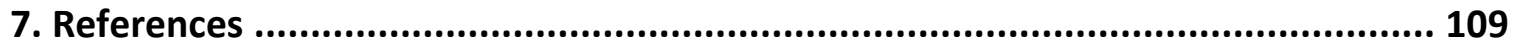

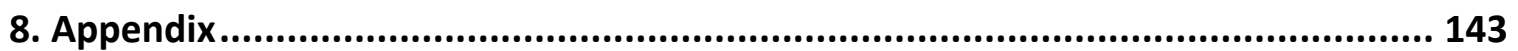

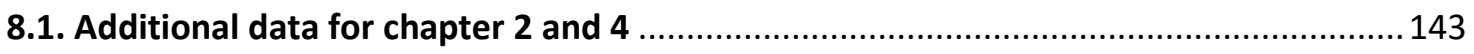

8.2. List of primers used for gene knock out in chapters $\mathbf{2}$ and 4. Blue and Red color letters are showing complementary regions with NAT sequence in PAG25. Black letters are complementary regions with upstream and downstream of target genes for knock out. Confirmation primers (Conf) are designed for gene replacement confirmation with NAT resistance marker.

8.3. 6-galactosidase quantification data for chapter 5. 6-galactosidase activity of candidate genes, selected from lift assay screening, was performed to confirm the reduction in the translation of 6 -galactosidase. The data was normalized to that of WT carrying either of the

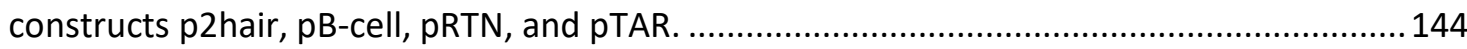

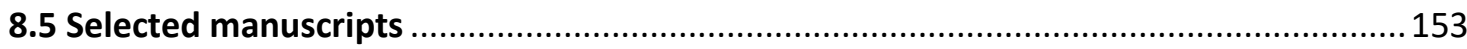




\section{List of tables}

Table 5.1. Candidate genes that seem to influence the expression of different 6 galactosidase constructs pTAR, pRTN, pBcell and p2hair. 


\section{List of figures}

Figure 1.1 Experimental approaches in systems biology.

Figure 1.2 A schematic representation of genetic interactions (GI) and noninteracting pathways.

Figure 1.3 A schematic illustration of eukaryotic translation step.

Figure 1.4 Diagram showing the step by step approach in this thesis.

Figure 2.1 Drug sensitivity analysis for different yeast strains using spot test analysis.

Figure 2.2 Quantitative analysis of drug sensitivity for different yeast strains.

Figure 2.3 Protein and mRNA content analysis.

Figure 2.4 B-galactosidase expression analysis in different yeast strains.

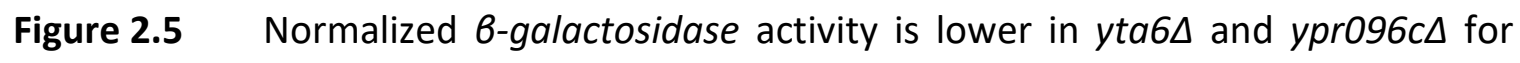
structured mRNAs.

Figure 2.6 Conditional nGIs for YTA6 and YPRO96C under $3 \mathrm{mM}$ concentration of LiCl.

Figure 2.7 Over-expression of YTA6 and YPR096C compensate for sensitivity of eap1S and $b c k 1 \Delta$ to $10 \mathrm{mM} \mathrm{LiCl}$.

Figure 3.1. Drug sensitivity analysis for different yeast strains using spot test analysis.

Figure 3.2 Quantitative analysis of drug sensitivity for different yeast strains.

Figure 3.3 Protein and mRNA content analysis. 
Figure 3.4 b-galactosidase expression analysis in different yeast strains.

Figure 3.5 Normalized 6 -galactosidase activity is lower in nam $7 \Delta$, pus $2 \Delta$ and $r p / 27 b \Delta$ for structured mRNAs.

Figure 3.6 nGls for NAM7, PUS2 and RPL27B.

Figure 3.7 Conditional nGls for for NAM7, PUS2 and RPL27B under $3 \mathrm{mM}$ concentration of $\mathrm{LiCl}$.

Figure 4.1 Schematic representation of the B-galactosidase mRNA designed to limit cytoplasmic translation.

Figure 4.2 mRNA content and b-galactosidase expression analysis of isolated mitochondria from different yeast strains.

Figure 4.3 Analysis of relative 8 -galactosidase activities analysis.

Figure 4.4 A model for the role of 3'-UTR of OXA1 gene.

Figure 5.1 An array of deletion set, showing yeast mutant strains carrying a plasmid with a $L a c Z$ cassette reporter gene.

Figure 8.1. The secondary structure of PGM2 5'-UTR. 


\section{Abbreviations}

\begin{tabular}{|c|c|}
\hline ATP & Adenosine Triphosphate \\
\hline $\mathrm{BD}$ & Bipolar Disorder \\
\hline cDNA & Complementary DNA \\
\hline NAT & Nourseothricin Sulfate (clonNAT) \\
\hline eEF & Eukaryotic Elongation Factor \\
\hline elF & Eukaryotic Initiation Factor \\
\hline FFE & Free Flow Electrophoresis \\
\hline GAL & Galactose \\
\hline $\mathrm{Gl}$ & Genetic Interaction \\
\hline GSK-3 & glycogen synthesis kinase-3 \\
\hline G418 & Geneticin \\
\hline IC & Initiation Complex \\
\hline IRES & Internal Ribosome Entry Site \\
\hline $\mathrm{B}$ & Lysogeny Broth \\
\hline
\end{tabular}




\begin{tabular}{|c|c|}
\hline $\mathrm{LiCl}$ & Lithium Chloride \\
\hline MAT & Mating type locus \\
\hline mtDNA & mitochondrial genome \\
\hline mRNA & messenger RNA \\
\hline ONPG & O-Nitro-Phenyl- $\beta$-D-Galactoside \\
\hline ORF & Open Reading Frame \\
\hline OXPHOS & Oxidative phosphorylation \\
\hline PABP & Poly A Binding Protein \\
\hline PCE & Post-Transcriptional Control Elements \\
\hline PCR & Polymerase Chain Reaction \\
\hline PIC & pre-initiation complex \\
\hline PIPE & Protein-Protein Interaction Prediction Engine \\
\hline PKC & Protein Kinase C \\
\hline PPI & Protein-Protein Interaction \\
\hline PSA & Phenotypic Suppression Array \\
\hline
\end{tabular}




\begin{tabular}{|c|c|}
\hline qRT-PCR & Quantitative RT-PCR \\
\hline ROS & Reactive Oxygen Species \\
\hline rRNA & Ribosomal RNA \\
\hline SC & Synthetic Complete media \\
\hline SD & Shine-Dalgarno \\
\hline SDL & Synthetic Dosage Lethality \\
\hline SDS-PAGE & Sodium Dodecyl Sulfate Poly-Acrylamide Gel Electrophoresis \\
\hline SGA & Synthetic Genetic Array \\
\hline SGD & Saccharomyces genome database \\
\hline TC & Ternary Complex \\
\hline mRNP & ribonucleoprotein mRNA \\
\hline mTOR & mammalian Target of Rapamycin \\
\hline tRNA & transfer RNA \\
\hline URA & Uracil \\
\hline UTR & Untranslated Region \\
\hline
\end{tabular}


X-gal bromo-4-chloro-3-indolyl- $\beta$-D-galactopyranoside

YPD Yeast extracts peptone dextrose media

4E-BPs elF4E binding protein

5' Cap 7-Methylguanosine (m7G) 


\section{Introduction}

\subsection{Systems Biology}

\subsubsection{Functional Analysis in Systems Biology}

Treatment for many diseases that so far have proven to be "incurable" including different types of cancer, diabetes, certain heart conditions and Parkinson's and Parkinson's-like diseases has formed a major focus of cell biology research. Numerous genes have been discovered linked to the onset of these complex diseases ${ }^{1,2}$ and much has been learned. To date, however, a feasible approach to untangle the problem of complex diseases has remained ambiguous. It is generally accepted that complex diseases are not linked to phenotypic consequences of one or a few genes, but rather a cascade of circumstances in a larger and dynamic system in association with a number of interconnected genes and factors that influence the onset and progression of these diseases. These genes are not connected to each other by a simple network but rather an interactive and dynamic web of interactions and feedback loops that are needed to mediate their activities as a system

1,3. Therefore, there is a need to investigate complex diseases in the context of such interconnected networks of interactions and as dynamic biological systems.

To better understand the balance between tens of thousands of compounds that work in harmony regulated by genetic information in the cell ${ }^{2}$ it is inevitable to investigate a biological cell as an integrated unit ${ }^{4}$. 
Studying the behavior of molecules and dynamic interactions of genes and pathways at a system-level is the first step of the journey to understand how single cells, tissues, and organs interact with one another, respond to internal and external stimuli and how the living system functions. In addition, systems biology allows us to investigate the function of genes or proteins in multiple pathways at the same time ${ }^{5}$. The idea of a single function for a protein has faded over the years. Now we know that a single protein can function in multiple pathways, can move from an organelle to another to participate in a completely different process and interact with different sets of proteins to perform various functions. For example, elF4E, one of the important factors in the initiation of the eukaryotic protein synthesis pathway, is not only crucial to translation but also can participate in transporting mRNAs from the nucleus to cytoplasm ${ }^{6}$. Systems biology can help us better investigate the various functions of proteins in a single cell or even in different tissues of a multicellular organism. Once we have a more clear understanding of the interaction networks for a target protein, we would be able to better realize the activity of that protein in a different context such as the onset and progression of different diseases ${ }^{3}$.

Systems biology received considerable attention after the development of certain tools including whole genome sequencing, DNA Microarray analysis, high throughput protein identification using Mass Spectrometry, and various proteomics approaches among others ${ }^{7}$. These tools allowed scientists to observe the structure and dynamic changes in the entire system at the same time ${ }^{4}$. Network models derived from such observations and analysis, helped in simplification of the connected function of proteins in the systems ${ }^{3}$. As Kitano explains in his review ${ }^{4}$, there are four main approaches to understand the 
connection of genes and proteins at system-level, the first approach is to investigate the genetic network of genes and pathways. The second is studying the system's dynamics over time under different conditions. Third, to see how the control mechanisms work to maintain the state of the cell and prevent any dysregulation. And forth is designing an acceptable model based on known facts and simulations ${ }^{4}$. Shown in Figure 1.1, a proposed algorithm for designing a systems-level approach is illustrated.

A big challenge in molecular systems biology is to identify the relevant information from a sea of data, much of which often contains a high occurrence of background noise and artifacts. An inherent disadvantage of large scale experiments and high throughout screens is that they generally suffer from high rates of false positives and negatives ${ }^{8-13}$. Consequently, analyzing data is a crucial step that should be taken into consideration really carefully ${ }^{14}$. The development of improved methods has significantly improved the quality of generated data ${ }^{15-19}$. Additionally, advanced bioinformatics tools have been developed to screen through large biological data in order to better separate false positives $^{9,20,21}$. 


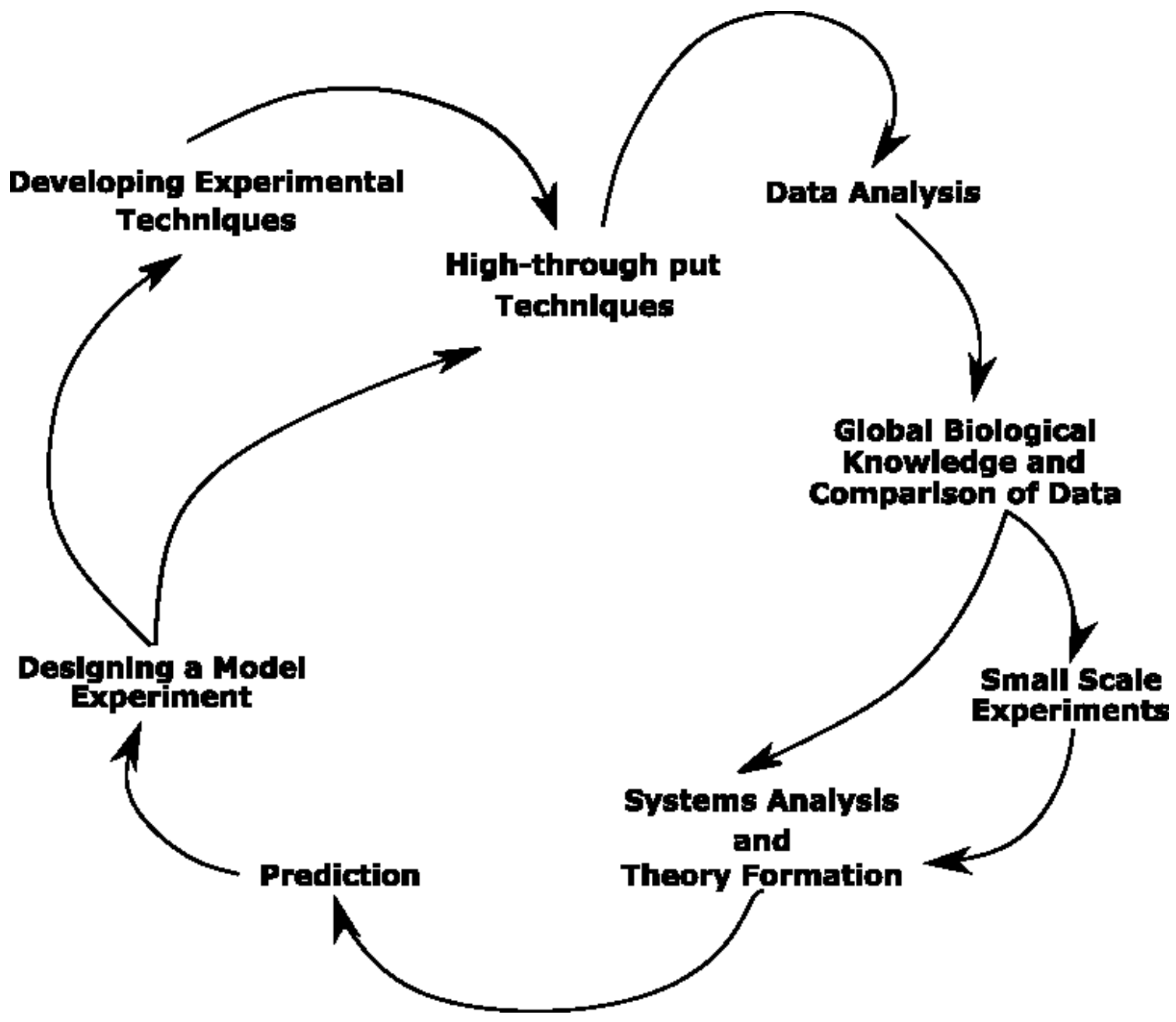

Figure 1.1 Experimental approaches in systems biology. The research starts by studying the literature and collecting data, analysis of the information and making predictions based on the available data. The next step is to design an experiment to test the hypothesis through high-throughput experiments and developing more efficient techniques to collect new data. The last step is to analyze the collected data from the experiment and compare it to the literature to confirm the hypotheses. Small scale (follow up) experiments can provide further support for the outcome of large scale experiments.

\subsubsection{Functional Genomics}

High-throughput techniques and specific small-scale experiments allow us to collect biological data for various biochemical networks within the cell, whereas mathematical modeling and bioinformatics tools are crucial for analyzing and making sense of the result 
$2,21,22$. Visualization of data constitutes a different challenge for which a number of bioinformatics tools have been developed ${ }^{23-25}$. In these tools, genes/proteins are often represented as nodes and the networks of genes and proteins are represented as edges, which often constitute physical or genetic interactions. Much of this data and elucidated networks could be used to further investigate gene functions in a systematic way. Functional genomics aims to investigate the novel function of genes and proteins using high-throughput techniques and genome-wide data analysis ${ }^{26-29}$.

The baker's yeast, Saccharomyces cerevisiae is a single-cell eukaryotic model organism that has been broadly used, not only to investigate various cell processes and pathways in molecular biology investigations ${ }^{28,30,31}$ but also as a model organism of choice in functional genomics, proteomics, and evolutionary genetics ${ }^{32-35}$. This model organism has a short life cycle, its genomic manipulation is fairly easy, possesses both haploid and diploid life cycles (aids in a number of high-throughput techniques), is the first eukaryote to have its genome sequenced, and genomics and proteomics annotations are available through different databases (SGD; www.yeastgenome.org, and https://yeast.biocyc.org/ $\left.{ }^{36}\right)$. Yeast is also a valuable asset for human-related genetic studies; there is a $30 \%$ homology between human and yeast genome, $87 \%$ of yeast protein domains are present in human proteome and nearly $50 \%$ of the genes involved in human heritable diseases have a homologous counterpart in yeast ${ }^{36-38}$. In a recent study, $47 \%$ of the yeast genes were replaced by their human orthologues and strains showed recovery from their deletion mutants; $53 \%$ of the genes involved in the translation pathway showed to be completely replaceable in yeast by human orthologs ${ }^{39}$. This is despite the fact that there 
were notable differences in gene sequences, splicing and protein assembly between orthologues genes ${ }^{39}$. Mercatanti et al (2017) analyzed 8078 missense mutations found in 31 cancer-related genes with homology in yeast, covering more than $50 \%$ of the missense variants causing cancer, making yeast-based functional assays a valuable source for cancer research ${ }^{38}$. This is in addition to the capability of evaluating yeast paralogues for therapeutic reasons.

Naturally, in the early days of systems biology, yeast was used as a tool for the development of high-throughput methods and large scale screenings ${ }^{36}$. To date, it continues to serve as a reliable resource for developing new and improved methods to systematically study a cell ${ }^{40-42}$. Many of these tools were developed to study proteinprotein interactions (PPI) ${ }^{16,19,43}$ whereas others are used to identify genetic interactions (GIs) ${ }^{18,44,45}$. Both PPI and GI data are heavily used to study functional genomics in order to assign novel functions to different genes under various conditions $27,31,34$. They are utilized in form of a "guilt-by-association" concept, where interacting proteins are thought to be functionally related ${ }^{46}$. For example, Krogan et al (2003) used PPI data to connect the Paf1 complex, associated with elongating polymerase activity, to histone methylation providing evidence for a connection between chromatin remodeling and transcription elongation ${ }^{32}$. Similarly, Butland et al (2007) connected the activity of bacterial Dead1 protein to translation initiation ${ }^{47}$. PPI data analysis continues to be an important tool for studying novel gene functions. For example, Omidi et al (2018) used PPI investigations to connect the activity of and previously uncharacterized proteins to the process of no-homologous end joining in yeast ${ }^{27}$. Similarly, Babu et al (2018) using a 
global PPI investigation approach, identified a number of proteins with novel roles in envelope protein complexes in bacteria ${ }^{48}$.

Similarly, GIs have been used extensively to elucidate novel gene functions. The following section focuses on the use of GIs in functional genomics.

\subsubsection{Genetic interaction}

As mentioned earlier, yeast has practical features that enables large-scale screening and investigate protein and genetic interactions in different pathways. System-level analysis of yeast with Saccharomyces Genome Project in 1996, identified nearly 6000 open reading frames (ORFs) ${ }^{49}$. This was followed by the Genome Deletion Project that lead to the development of yeast non-essential Gene Deletion Array, also known as loss-offunction in which more than $96 \%$ of the yeast genes were each successfully deleted providing scientist with an important tool to study gene functions ${ }^{50}$. Gene deletion mutant library has been used for genome-wide investigation of pathways and gene functions in a living cell and under different conditions like stress or target pathway of different compounds $31,33,34,44,51$.

The functional relationship between genes and pathways can be described based on genetic interactions ${ }^{52}$. $\mathrm{Gl}$ is loosely defined when the phenotype of a double-mutant is unexpectedly different from that of single mutants ${ }^{53}$ (Figure 1.2); it helps to explain a higher level of association between the functions of two genes in parallel pathways (epistasis) or in the same essential pathway ${ }^{53}$. Negative $\mathrm{Gl}$ is assumed when an 
aggravating phenotype is observed, for example the fitness of cells measured by the size of the colony formed becomes significantly smaller than expected colony size of single mutants (Figure 1.2B). In this case, Gls are said to have synthetic sick phenotype or, in extreme cases, synthetic lethality. Similarly, positive genetic interaction refers to cases where double mutants have alleviating, or in this example bigger colonies, than expected

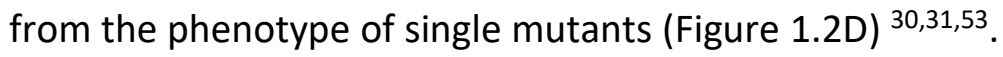

Yeast is considered an ideal tool for investigating systematic Gls because $80 \%$ of its genes are non-essential, and colony size can be used as a measure of fitness. Availability of two mating type makes systematic generation of double mutants a relatively easy task ${ }^{7}$. High throughput generation of double mutants in yeast is possible through a technique called Synthetic Genetic Array (SGA) analysis. In brief, a single gene deletion marked with a selection marker in alpha mating type is crossed to the array of single gene deletion mutants of a-mating type carrying a different selection marker. This procedure is made possible using a replica-pinning approach where floating pins, usually in a 384 format, are used to deliver yeast colonies from one plate to another ${ }^{24,53}$. After a few rounds of selections that includes sporulation, the progeny of a-mating type that carry double gene deletions is selected. The fitness of double gene deletion mutants is then analyzed using their colony size. The bigger the colony the higher the fitness. The size of the colonies can be automatically measured and analyzed using various software's ${ }^{24,54,55}$.

A second type of negative $\mathrm{Gl}$ is termed dosage lethality. In this case, the overexpression of one gene results in an unexpected aggravating phenotype in a gene deletion background (Figure 1.2E) ${ }^{56}$. The high throughput method to study such interactions in 
yeast is made possible by a modified SGA approach. In this case, after mating and sporulation steps, the "a" mating type progeny containing a non-essential gene deletion that carries an overexpression plasmid for the query gene is selected. As above, colony size can be used as a measure of fitness. 
A) Non-interacting pathways

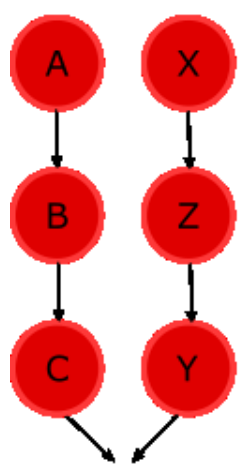

Viable

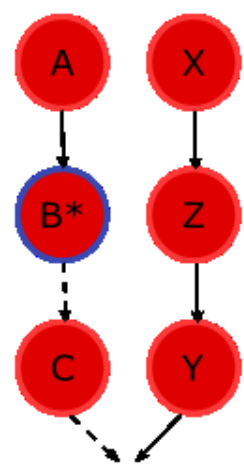

Viable

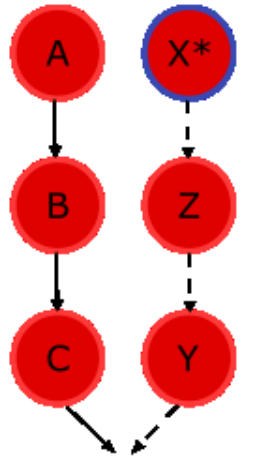

Viable

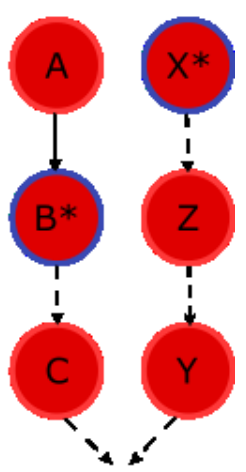

Viable

B) Genetic Interaction pathway

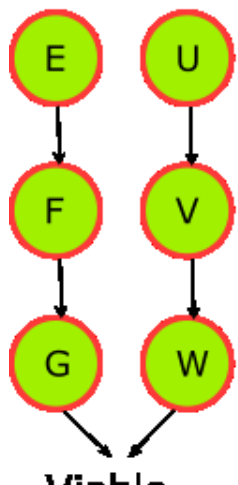

Viable

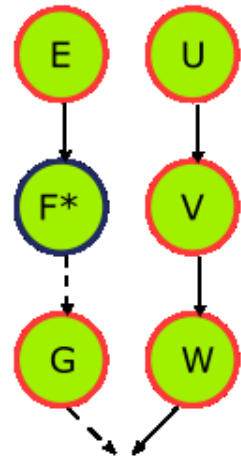

Viable

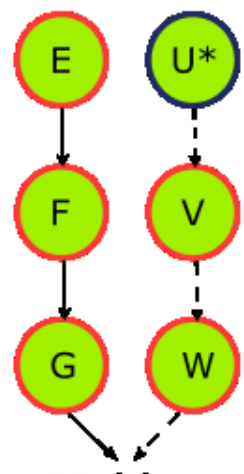

Viable

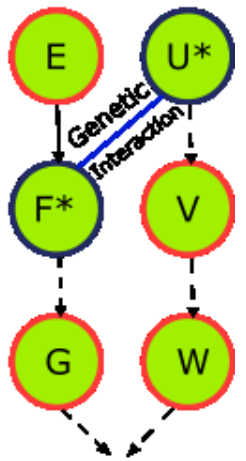

Inviable or sick

C) Chemical Genetic Interaction

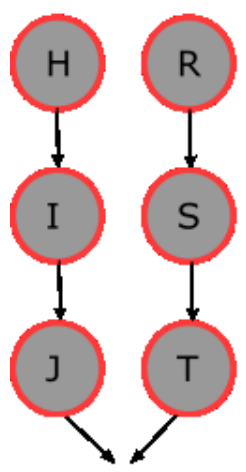

Viable

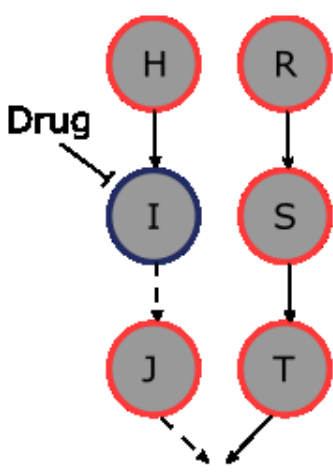

Viable

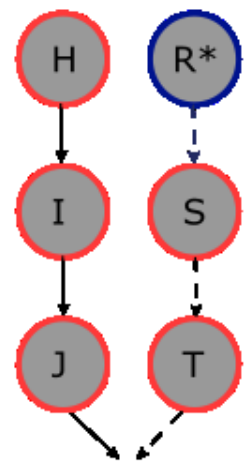

Viable

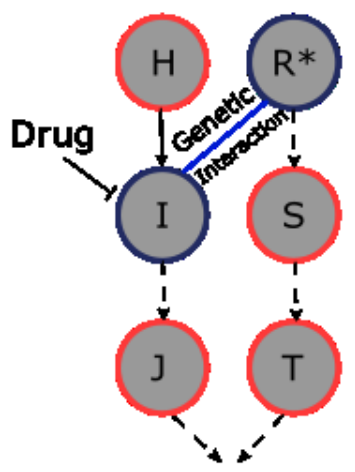

Inviable or sick 


\section{D) Positive Genetic Interaction pathways}
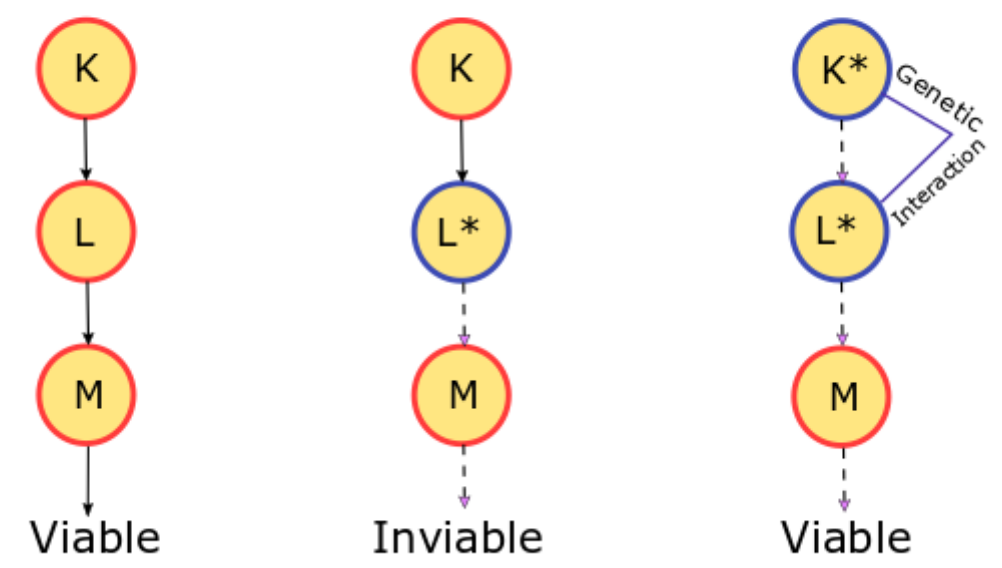

\section{E) Synthetic Dosage Lethality}
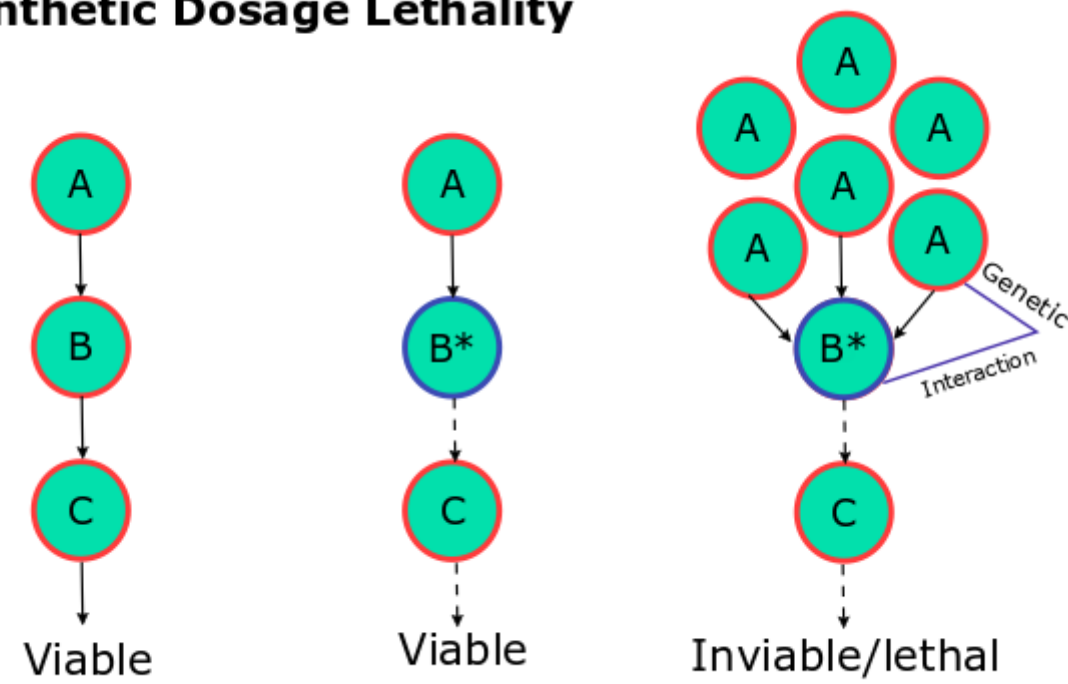

Figure 1.2. A schematic representation of genetic interactions (GI) and non-interacting pathways. A) The two pathways are independent of each other. Double mutants are viable. B) Two pathways are parallel and compensating. Double gene deletion leads to sickness or lethality of the cell, (negative GI). C) A gene deletion mutation in (B) is replaced by a chemical that might deactivate the gene product. This form of $\mathrm{Gl}$ is called chemical genetic interaction. D) In positive $\mathrm{Gl}$, the deletion of a second gene reverts the sick/inviable phenotype of the first mutation. The second gene is often upstream of the first in the same pathway. E) Dosage lethality interaction is seen when over-expression of one gene in a single mutant background proves to be lethal. "*" means the gene is deleted. 
Once the GI profile(s) for a target gene is determined, potential gene functions may be assigned. For example, it is expected that genes that are associated with protein biosynthesis would form Gls with protein biosynthesis genes ${ }^{31,34}$ whereas those associated with DNA damage repair would interact among themselves ${ }^{28,57}$. Generally, it is accepted that genes that function on parallel compensating pathways can often form negative GI when both are deleted. Dosage lethal genes, on the other hand, are thought to be on the pathway where the overproduction of a product made by one gene may prove to be lethal to the cell.

Another form of $\mathrm{Gl}$ can be studied under the treatment of chemicals, serving as modulators of genes. This area of research is often called Chemical Genomics ${ }^{7,58}$ (Figure 1.2C). One way to study chemical genomics is using a chemical as a second mutation in a cell. In this case, a gene deletion mutant is used as the first alteration and the mode of action of a chemical is used as the second alteration. The chemicals with known mode of actions can be used to predict the function of an interacting gene in a parallel pathway 33,59-62. Sub-inhibitory concentration of chemicals may also be used to further study Gls ${ }^{63-65}$. In this case treatment of double-mutants under the sub-inhibitory concentration of the chemical can generate new Gls forming conditional Gls ${ }^{7,33}$. In general conditional genetic analysis (under stress or drug), or conditional SGA, allows scientists to identify genes that are activated or induced under specific conditions revealing the novel function of genes and pathways. Based on the fact that certain pathways and genes are only 
expressed under specific stimuli, conditional SGA can be a valuable method to study novel gene functions $33,34,66$.

\subsection{Translation}

\subsubsection{Translation pathway and the significance of studying it.}

Protein synthesis is a highly conserved process that makes polypeptide chains from genetic codons transcribed on mRNA templates ${ }^{67}$. Proteins are important for almost all cellular reactions, structural molecules, in transportation, cell signaling and other important mechanisms ${ }^{68}$. Ribosomes are complex macromolecules that constitute the "machinery" of the translation process. In addition to their role in translation, ribosomes are also involved in engaging kinases and consequently signaling pathways ${ }^{69}$. Cell signaling pathways affect translation regulation by modifying the activity of translational factors through phosphorylation by the target of rapamycin (TOR) and mitogen-activated protein kinase (MAPK), among others ${ }^{6,70}$. Protein synthesis consumes up to $50 \%$ of the cell energy and in yeast equivalent to $30-40 \%$ of the cytoplasm volume is occupied by ribosomes (approximately 200,000 ribosomes) during growth stages ${ }^{68}$. Ribosomes are considered the core components of protein synthesis, consisting of two main subunits, small subunit with a crucial role in decoding the genetic code, and large subunit that contributes to the formation of polypeptide bonds. Ribosomal subunits are composed of proteins and rRNAs; rRNAs form the main enzymatic and structural component of the complex ${ }^{71,72}$. 
The translation process in eukaryotes and prokaryotes includes three major steps: initiation, elongation, and termination. One of the main differences is that in prokaryotes protein synthesis and transcription are taking place in the cytoplasm and can be coupled together at the same time, whereas in eukaryotes transcription is taking place in the nucleus and translation is in the cytoplasm after maturation of mRNA through splicing, addition of $5^{\prime}$ cap and poly(A) tail and transport out of nucleus ${ }^{67}$.

Translation initiation in prokaryotes starts with binding of the small subunit of ribosome (30S) consisting of the $16 \mathrm{~S}$ rRNA and 21 proteins, to the unstructured region on mRNA called Shine-Dalgarno (SD), complementary to $16 \mathrm{~S}$ rRNA sequence $5^{\prime}$ to the start codon $68,72,73$. The recognition of the translation initiation site in prokaryotes is generally based on an RNA-RNA interaction ${ }^{67}$. Then large ribosomal subunit (50S), consisting of $5 \mathrm{~S}$ and 23S rRNAs and 33 proteins, binds to small subunit to form 70S initiation complex (IC) to enter elongation step in prokaryotes ${ }^{72}$.

In elongation step, peptidyl transferase activity of large ribosomal subunit synthesizes peptide bonds between corresponding amino acids, based on triplet nucleotide codons of mRNA. This is done with the help of elongation factors and other elements ${ }^{67,68}$. When the termination site is reached, at stop codons, instead of amino acid incorporation, termination factors enter the ribosome and induce dissociation of the polypeptide chain and ribosomal subunits from mRNA ${ }^{67}$.

The translation procedure in eukaryotes follows the same overall concept with a bit of variation throughout the process. The first thing to notice is the larger size of the 
ribosomal subunits, the $40 \mathrm{~S}$ small subunit that consists of $18 \mathrm{~S}$ rRNA, and the 60S large subunit composed of $5 S, 5.8 S$ and $25 \mathrm{~S}$ rRNAs, 80 proteins ( 79 in yeast), that together form the 80 S ribosomal complex $67,69,71,72$. Recognition of mRNA and initiation step differs in the eukaryotic system compared to prokaryotes. Eukaryotic mRNAs undergo a series of modifications to form mature mRNAs before translation can take place. In general mRNA splicing, addition of 7-Methylguanosine $\left(\mathrm{m}^{7} \mathrm{G}\right)$ at $5^{\prime}$ end of mRNA ( $5^{\prime}$ cap) and poly $(A)$ tail at their $3^{\prime}$ end ${ }^{67}$ lead to the maturation of mRNAs. In brief, the small subunit of the ribosome with the help of initiation factors recognizes the 5' cap and brings the initiator methionyl-tRNA $A_{i}$ to the pre-initiation complex (PIC). The process starts by scanning for the correct AUG start codon ${ }^{67}$.

Translation initiation is regulated by IF1, IF2 and IF3 in prokaryotes and elF1, elF1A, elF2 (subunits $\alpha, \beta$ and $\gamma$ ), elF2A, elF3 (subunits a-m in mammals and $a, b, c, g, h$ and $j$ in yeast), elF4F (subunits 4A, 4E and 4G), elF4H, elF4B, elF5, elF5A, elF5B and elF6, among others, in eukaryotes. Not only are these factors are important in starting the translation process but they are also important in controlling translation efficiency and are known to be controlled by various regulatory pathways ${ }^{73-76}$. Elongation step is highly conserved between eukaryotes and prokaryotes and termination, although relatively conserved, is promoted by different proteins.

Although much has been learned about these steps, there appears to remain other proteins and complexes affecting translation efficiency, and especially the regulation of translation needs to be further investigated. To give an example, the role of elF2A is known to induce binding of Met-tRNA $A_{i}$ to $40 \mathrm{~S}$ ribosomal subunit, but Dmitriev and et al., 
proposed the role of another initiation factor, elF2D to encourage the binding of tRNA to ribosomal P-site in absence of GTP ${ }^{77}$. Or the clear function of elF5A was known to be essential for translation initiation, but recently it was proposed its role in translation elongation and termination ${ }^{78}$. Also, recently it has been shown that translation regulation is controlled not only by translation factors but also by ribosomal proteins, known to have structural features involved in translation regulation. Protein components of the ribosome have been shown to engage in transcription, DNA repair and life span in yeast 79. The role of elF4A, the main RNA helicase in translation is not clear either. Studies have shown that probably different types of RNA helicase are regulating different mRNAs in different tissues with or without correlation with elF4A ${ }^{76,80}$. The list of proteins affecting both prokaryotic ${ }^{81-84}$ and eukaryotic translation ${ }^{9,34}$ continues to grow. There is a lot more to be understood about this process and its regulation in addition to its cross-talk with other processes. The main focus of the current thesis is to investigate new RNA helicases and associated factors, involved in this pathway, specifically those associated with the translation of highly structured mRNAs.

In addition to the central role of translation in the process of gene expression, a number of diseases have been linked to protein biosynthesis dysfunction, making translation pathway a major target for treatment ${ }^{6}$. Not only the translation machinery but also the dysregulation of genes involved in translational control has been shown to affect oncogenic pathways and consequently lead to tumor development ${ }^{6,85}$. For example, elF4E is considered as one of the important targets of cell signaling pathways to induce growth and proliferation. It is thought that elF4E malfunction is connected to 
abnormalities in cell growth as seen in various cancers including prostate, breast, skin, lung, colorectal, bladder and cervical cancers ${ }^{6,86}$. elF4E has also recently been connected to autism spectrum disorder ${ }^{87}$. elF4A is overexpressed in melanoma cells where elF3f is excised (subunit of elF3) 86,88 . elF3 is overexpressed in breast cancer and elF4E overexpression is linked to lung carcinoma ${ }^{85}$. elF4A inhibition (with Hippuristanol) is shown to delay Alzheimer's disease progression in early stages of recognition when cells are under oxidative stress ${ }^{89}$. Also, the inhibition of elF4A can selectively reduce the translation rate of genes involved in the progression of Alzheimer's disease including Abeta (Amyloid $\beta$ peptide) and tau (microtubule-associated protein) genes that happen to have highly structured $5^{\prime}$-UTRs but not the housekeeping genes ${ }^{89}$. The connection of translation to various diseases provides us with additional reasons for investigating this process and its regulations.

\subsubsection{Translation Initiation step}

The initiation step of translation is the most regulated step of the pathway ${ }^{6,76,90}$. In the pre-initiation of cap-dependent eukaryotic translation, Met-tRNA $A_{i}$ promoted by elF1, with eukaryotic initiation factor 2 (elF2), and GTP forms a complex called ternary complex (TC). TC binds to the small subunit of ribosome (40S), making 43S PIC that includes other initiation factors elF1, elF1A, elF5 and elF3 to scan for the start codon ${ }^{74,76}$. elF1 and elF1A are involved in the opening of mRNA and tRNA binding grooves for scanning and detecting the start codon as well as preventing the association of large subunit at this stage ${ }^{72}$. The role of elF6 is not well understood but there are suggestions that it might prevent the early recruitment of large subunit to the PIC ${ }^{72}$. Before scanning, PIC binds to $5^{\prime}$ cap of 
mRNA with the help of elF3, elF4H, elF4B, and elF4F, that consists of elF4E as cap-binding protein, elF4A an RNA helicase and elF4G as scaffolding protein to bind the complex to poly (A) binding protein (PABP) at $3^{\prime}$ end of mRNA (Figure 1.3) ${ }^{75,90}$. elF4A, is not the only helicase enzyme involved in this complex, but it is suggested that other enzymes like Ded1 may also bind to this complex and function as helicases ${ }^{91}$. Binding of PIC to PABP makes a stable circular ribonucleoprotein mRNA (mRNP) to facilitate the restarting of translation on the same mRNA ${ }^{76}$. mRNP and elF3 allow the association of 435 complex and helicase activity of elF4A helps unwind the secondary structure with the help of elF4B to stimulate its ATP hydrolysis and start scanning ${ }^{76,92}$. elF3 is a large complex of 13 different proteins in mammals and six in yeast that binds to mRNA and stimulates PIC assembly for proper scanning ${ }^{93}$. elF4F complex, plays crucial roles in recruiting 435 subunit to mRNA ${ }^{90}$. For highly structured untranslated regions (UTRs) mRNAs, the need for elF4A and elF4F is higher ${ }^{76,94}$. Base-pairing of Met-tRNA ${ }_{i}$ anticodon and AUG in the P site of small ribosomal subunit induces the release of elF2 and GTP-activation of elF5, the release of elF2,GDP and other elFs including elF1 of the complex and consequently association of $60 \mathrm{~S}$ ribosomal subunit by elF5 and formation of 80 S IC followed by the entry of machinery into elongation step $76,90,95$. 


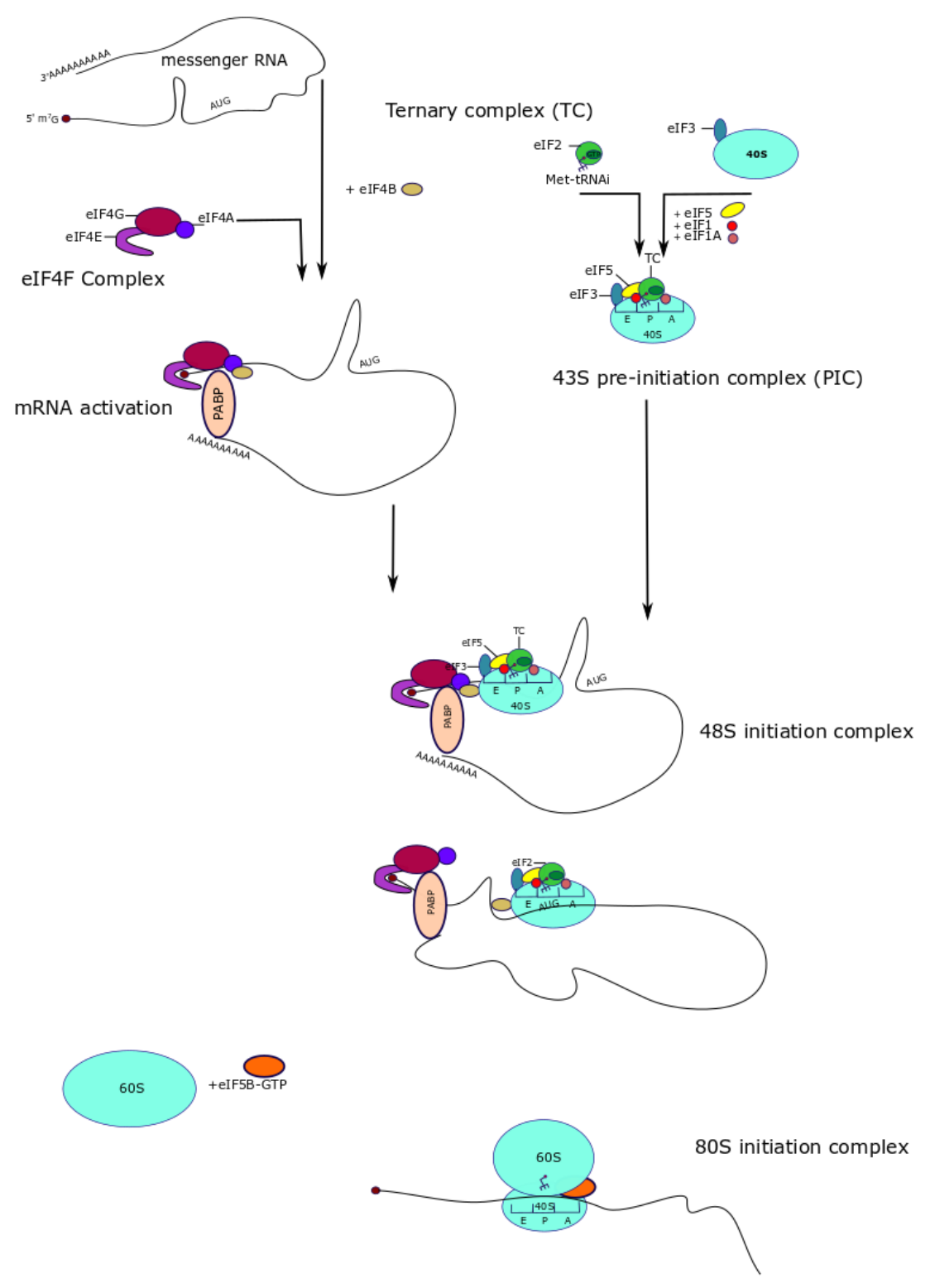

Figure 1.3. A schematic illustration of the eukaryotic translation pre-initiation step. The pre-initiation step starts with mRNA activation when the elF4F complex binds to $5^{\prime}$ cap of mRNA. Then TC carrying the initiator methionine amino acid binds to the $40 \mathrm{~S}$ subunit of the ribosome along with other initiation factors to form PIC. The recruitment of PIC on activated mRNA forms $48 \mathrm{~S} I \mathrm{C}$, ready for scanning the mRNA in search of start codon. When AUG is detected eIF2 is induced to dissociate from the IC along with other translation initiation factors. This will allow the $60 \mathrm{~S}$ ribosomal subunit to bind to the $40 \mathrm{~S}$ subunit with the help of elF5B to form 80 S IC ready to move to the elongation step. 
In the prokaryotic system, the initiation step is regulated through three main factors, IF1, IF2, and IF3 to find the right start codon and recruit initiator tRNA into the P-site of small ribosomal subunit ${ }^{72}$.

\subsubsection{RNA helicases}

RNA helicases are not only involved in unwinding of secondary structure (also known as hairpins or sometimes inhibitory structures) on mRNAs during translation but also are involved in transcription, mRNA splicing, mRNA export, mRNA storage, and mRNA decay pathway ${ }^{91,96,97}$. DEAD box helicases, a common family of conserved helicases that contain DEAD motif are involved in unwinding RNA hairpins, remodeling ribonucleoprotein complexes for better scanning and recruitment of macromolecules of translation machinery on mRNA, spliceosome complexes and mRNA nuclear export ${ }^{98}$. In fact, in many cases, it seems that the fate of the mRNA, to be translated, degraded or stored depends on different types of helicases ${ }^{99}$.

elF4A, the main helicase for translation initiation belongs to the DEAD-Box family of RNA helicases that have a highly conserved domain called RecA. This domain forms a stabile structure after binding to ATP and RNA, promoting the helicase activity ${ }^{92}$. elF4A is an important component of the elF4F complex in the cap recognition in proliferating cells. This fact makes helicases possible oncogene and potential targets of anti-proliferative drugs. Pateamine A, Hippuristanol and Silvestrol are the inhibitors of elF4A discovered over the past decade to control translation initiation in growing cells ${ }^{100}$. 
elf4A is generally thought to be the main RNA helicase in the translation pathway responsible for unwinding secondary structure of mRNA in the initiation step to allow scanning for start codon AUG. It has been shown that multiple molecules of eIF4A can be recruited to an individual mRNA in the PIC ${ }^{76}$. However, it is not clear if this factor (elF4A) continues unwinding the mRNA along the way or does other helicases/proteins function or associate with elF4A in the process. It is thought that in different body tissues, different helicases replace eIF4A in the IC ${ }^{80}$.

DED1 and its close homolog DBP1 are the two of the reported DEAD-Box RNA helicases in yeast with homology to $D D X 3$ in mammals. DED1 is involved in mRNA splicing and its depletions affect the splicing as well as the global translation rate ${ }^{91}$. It was recently observed that DED1 methylation affect the regulation of specific mRNAs ${ }^{101}$. Its mammalian homolog, $D D X 3$ is also involved in transcription, mRNA export, and translation. Its role in translation has been connected to Internal Ribosome Entry Site (IRES) regulating cap-independent translation regulation ${ }^{91}$. More recent studies proposed the cap-dependent regulation of DDX3 for specific mRNAs with structured 5'UTRs happens to be close to $5^{\prime}$ cap, suggesting their role in recognition of cap and cooperating with elF4A to unwind and scan the transcript ${ }^{98}$.

Another group of DEAD-Box helicases influence the translation of those mRNAs that contain post-transcriptional control elements (PCE) on their $5^{\prime}$ termini ${ }^{96}$. It was observed that in some cases when the ribosome is stalled on mRNA and scanning has been interrupted, specific sequences in 5'-UTR region of mRNAs, which do not have IRES characteristic, will be recognized by this type of helicases. DHX9 known as RNA helicase A 
is an example of this group of helicases in humans ${ }^{96}$. It was proposed that RNA helicase A is important host factor for the proper translation of HIV-1 and a possible target for therapeutic purposes ${ }^{98,102}$. Helicases modify RNA-RNA and RNA-protein interaction to regulate translation. It remains a possibility that there are other proteins involved in the translation of specific types of mRNAs specifically for the ones with long structured 5'UTRs. A major challenge in the coming years will be to identify the substrates that are selectively regulated by each RNA helicase at each step of gene expression. It has already been shown that some helicases, including $D D X 3$ in human, are regulating specific transcripts, containing PCE elements, which cannot be modulated by other enzymes like elF4A ${ }^{98}$ and that they seem to be important in ribosome recruitment before $43 \mathrm{~S}$ subunit starts scanning by remodeling the mRNA ${ }^{98}$.

Advances in the field of RNA structure analysis will improve our understanding of how RNA helicases are potentially linked to various RNA structures. It was observed that in specific types of cancer overexpression of elF4A, upregulate translation of genes involved in cell proliferation and growth happens to have highly structured $5^{\prime}$ UTRs ${ }^{89}$. It is also important to note that small molecules can be developed to target the enzymatic activity of RNA helicases that would not only be useful to dissect the cellular functions of these factors, but also might possess therapeutic potentials, as several RNA helicases are involved in a wide array of diseases.

In humans, DHX29 is an important RNA helicase that can regulate translation initiation. It's malfunction or downregulation affects the efficiency of translation and impedes cancer cell growth in vitro ${ }^{90}$ making it a suitable target for cancer therapy. Other helicases 
like $D D X 3$ are shown to interact with elF4A and other initiation factors. These interactions may explain the mechanism by which such helicases affect translation regulation ${ }^{91}$. The existence of other helicases that can affect translation implies that there may exist other undiscovered helicases or helicase-affecting elements that can also influence mRNA translation ${ }^{91}$.

\subsection{Translational control}

\subsubsection{Translation control in gene expression}

Regulation of gene expression is taking place at different levels starting from chromatin remodeling, transcription, mRNA maturation, splicing, mRNA export, followed by translation and post-translational modifications. mRNA content does not necessarily reflect the level of translation ${ }^{6}$. Translation control is considered an important step in determining the rate of protein accumulation. Also, is one of the fastest responses to stress is thought to be at translation step, where the cell can quickly regulate protein synthesis to reduce/induce protein production in response to stimuli ${ }^{103}$.

Signaling pathways are known to be important in affecting the activity of translation factors in response to internal and external stimuli ${ }^{70}$. Translation control is important in many cellular processes including cell metabolism and proliferation and is shown to be connected to certain diseases including cancer and ribosomopathies, a class of inherited diseases caused by a malfunction in the component of ribosome structure like dyskeratosis congenita ${ }^{85}$. It is important to investigate and understand translation control 
and the corresponding upstream and downstream pathways to better realize the biology of the mentioned diseases ${ }^{85}$ and discovering potential therapeutic targets to tackle different diseases ${ }^{104}$.

As mentioned earlier, protein synthesis requires a great deal of cellular resources (energy). Naturally regulating translation at the initiation step, is the most cost-effective measure to regulate protein synthesis ${ }^{68}$. A number of key parameters for the regulation of eukaryotic translation initiation relies on certain components on mRNA including cisregulatory regions on 5'-UTR and 3'-UTR of different mRNAs, 5' cap structure, the length of mRNA, secondary structure of 5'-UTR, the Kozak sequence around AUG, poly(A) tail, uORFs, and trans-acting elements in addition to the availability of initiation factors, regulatory proteins and microRNAs ${ }^{95,105}$. In prokaryotes, the regulation seems a bit simpler and depend on the length of mRNA, SD sequence, non-SD translation enhancers 106-108 and initiators ${ }^{109}$, stability of secondary structure at both 5'- and 3'-UTRs, length of coding region among others ${ }^{110}$.

Translational control happens either at a global level for most mRNAs, or it applies to specific mRNAs with specific features ${ }^{68}$. In both scenarios, the goal is to change protein production or induce translation of mRNAs important to cell function under different conditions. Four general elements are thought to determine the global rate of translation, the mRNA level, abundance of the ribosome, rate of elongation and the activity of translation machinery (for example, phosphorylation of translational components) ${ }^{68,111}$. For more specific regulation, there are two general approaches to control the initiation step, regulation of translation initiation factors and utilizing cis-regulatory elements on 
mRNA, modulated by proteins or small RNAs ${ }^{112}$. At least 12 initiation factors are directly involved in the control of translation initiation step and most of them are targets of translation regulation ${ }^{76}$. Initiation factors, with mRNA binding features, can be phosphorylated, affecting translation and growth of the cell. elF2, elF4E, elF4G, elF4B and elF3 (elF4A in plants) ${ }^{68}$ are mostly regulated by mTOR and MAPK signaling pathways ${ }^{70}$. elF4E in the elF4F complex plays an important role in cap-dependent translation initiation where it competes with elF4E binding protein (4E-BPs), a substrate of mTOR pathway in binding to $5^{\prime}$ cap ${ }^{70,113}$. S6Ks form another group of translational repressors of mTOR pathway that regulate ribosomal protein S6, elF4B and programmed cell death 4 protein (PDCD4) ${ }^{70,114}$. mTOR pathway not only has a negative regulatory effect on translation but also by phosphorylating elF4B and elF4H, it can improve the helicase activity of elF4A. When it binds to PDCD4, elF4A loses its affinity to elF4G causing reduced cap-dependent translation ${ }^{114}$. The second regulation approach relies on $5^{\prime}$-UTR features of mRNAs that are important for both the recruitment of translation initiation factors and the rate of translation ${ }^{115}$. The most common cis-regulatory elements are 5' cap, hairpin and IRES structures, upstream open reading frames (UORF) and poly(A) tails ${ }^{112,116}$. uORFs are an important translation control in response to stress. It is thought that this mode of translation regulation affects $40 \%$ of all mammalian mRNAs ${ }^{117}$. Under certain stress conditions, uORFs are translated and the main ORF reading (the downstream one) is inhibited mostly through non-canonical initiation from uORF start codon and termination and dissociation of translation machinery before it reaches the main ORF ${ }^{117,118}$. Recently it was discovered that apart from uORFs role in inhibiting the translation of the main ORF, 
in some cases they can recruit translation initiation factors more efficiently on mRNA and hence promote the translation of the main ORF. In this manner, in response to stress, uORFs can both downregulate or upregulate the translation of certain genes ${ }^{117,119}$.

In contrast to the canonical mode of translation where the machinery recognizes the $5^{\prime}$ cap and starts scanning for start codon, using alternative strategies, 5\%-10\% of mRNAs can be recognized directly by the $40 \mathrm{~S}$ subunit of the ribosome. Some of these mRNAs contain specific structural elements called Internal Ribosome Entry Sites (IRES). IRESs are most widely used by RNA viruses to take advantage of the host cell translation machinery when general translation might be compromised. Certain proteins involved in cell growth and proliferation as well as those involved in the regulation of apoptosis, have been reported to use IRES-mediated translation $74,120,121$ when the canonical translation is inhibited.

Ribosome shunting ${ }^{122}$, tethering ${ }^{123}$, translation enhancers ${ }^{124,125}$, TISU element for short $5^{\prime}$-UTR mRNAs ${ }^{95,123}$ and poly(A) leader can also regulate translation ${ }^{74}$.

An interesting form of translation regulation is seen on specific mRNAs with $5^{\prime}$-UTR that carry poly $(A)$ regions. This mode of regulation is often observed when elF3 and elF4F are not readily available. These poly $(A)$ regions are often found just before the initiation codons and they appear to attract the $40 \mathrm{~S}$ ribosomal subunit to associate with mRNA and form an IC in an ATP-independent manner ${ }^{74}$.

In prokaryotes, translation is most commonly regulated directly by blocking the accessibility of machinery to the initiation site ${ }^{73}$. The strength of the complementary base 
pairing of the SD sequence with the $3^{\prime}$ end of the 16S rRNA is an important feature of translation regulation. Similarly, the availability of enhancer sequences and other translation initiation sequences to base pair with different regions of $16 \mathrm{~S}$ rRNA also seem to play a role in the initiation of translation ${ }^{107,126,127}$. mRNA-16S rRNA base pairing can be regulated by the structures within the mRNA or through mRNA-binding proteins ${ }^{73}$. Ribosome occupancy and density in the cell also play an important role in prokaryotic translation regulation ${ }^{110}$.

Translation regulation in mitochondria resembles those of prokaryotes. The Mitochondrion is known to have its own genomic information (known as mitochondrial genome (mtDNA)). However, approximately $95 \%$ of the proteins involved in mitochondrial pathways have nuclear origins including those affiliated with oxidative phosphorylation (OXPHOS), which is the main pathway for producing cell energy ${ }^{128}$. These mRNAs are directed to the vicinity of mitochondria for translation ${ }^{128-130}$ along with some tRNAs and other RNAs including rRNAs and InRNAs ${ }^{130}$. Some tRNAs are interchangeable between cytoplasm and mitochondria and some are specific to mitochondria ${ }^{130}$. mRNA transport into mitochondria is thought to be based on sequential interaction of mRNA with directing enzymes like Eno2P and pre-LysRS in yeast ${ }^{130}$. For rRNA import, both sequential interaction and membrane proteins are crucial for proper transfer into mammalian mitochondria ${ }^{130}$. Some mitochondrial mRNAs like COX2 have $5^{\prime}$ upstream regulatory regions that promote their translation in yeast mitochondria ${ }^{129}$. Translation regulation in mitochondria is proposed to be under indirect regulation of cytosolic translation ${ }^{128}$. 


\subsubsection{Translation control of mRNAs with structured 5'-UTRs}

In eukaryotic cells, translation control can regulate cell development, growth, tumorigenesis, differentiation, cell proliferation and protein production $6,67,90,131$. One of the cells fastest response to stress and apoptosis relies on translation control at the initiation step ${ }^{121}$. Translation initiation is considered the main target for translation control $67,76,96,115$. Other mechanisms including frameshifting and ribosomal stalling during the elongation step, also play roles in protein synthesis regulation ${ }^{73}$. Dysregulation of translational control is connected to many diseases including different types of cancers ${ }^{131}$. Commonly, such dysregulation is observed at the initiation step where the $40 \mathrm{~S}$ subunit is recruited to mRNAs ${ }^{104}$. elF4F plays an important role in regulating the initiation step and its components have become important targets for therapeutic purposes ${ }^{104}$. elF4E, one of the components of eIF4F is the cap-binding protein that under stress condition can be inhibited by phosphorylation of $4 \mathrm{E}-\mathrm{BP}^{6}$. This regulation is thought to affect so-called elF4E-sensitive subset of mRNAs that carry long and structured $5^{\prime}$-UTRs ${ }^{70}$. It was observed that mRNAs coding for proteins involved in proliferation, oncogenicity, and cell survival seem to carry such features and are regulated by the mentioned signaling pathway ${ }^{70}$.

The structure of mRNAs in eukaryotes is an important feature of translation regulation. these features include the 5' cap structure, sequences flanking AUG start codon, the position of AUG from $5^{\prime}$ end the secondary and tertiary structures within mRNA leader sequences ${ }^{73}$. In mRNAs with long $5^{\prime}$-UTRs the role of elF1, elF1A, DED1, elF2B, elF3, and

elF5 seems to be more important for proper scanning ${ }^{93,131}$. Some mRNAs have cis-acting 
sequences, mostly at their 5'-UTRs, that attract different trans-acting proteins and small regulatory RNAs to influence translation regulation ${ }^{105,112}$. It has been shown that the free energies of secondary structure on 5'-UTR of mRNA does affect the translation rate ${ }^{105}$. Such structures are often subjected to unwinding by helicases under different conditions. mRNA structure is not only crucial in translation initiation rate but it can also affect elongation and termination ${ }^{67}$; it is thought that RNA helicases might be required to unwind different structures along the way ${ }^{132}$. The shape of mRNA has also been connected to stress granule assembly. This is specifically relevant to mRNAs with long 5'UTRs that under stress conditions can be modified through cell cycle regulatory proteins such as WHI3 ${ }^{133}$.

In addition to elF4A other DEAD/DExH-Box RNA helicase, including DED1 and DBP1 in yeast and DDX3 and DHX29 in mammalian, are involved in unwinding and scanning steps of initiation for mRNAs with highly structured 5'-UTRs, mostly involved in cell growth, proliferation, and morphogenesis $76,91,134$. Confocal microscopy analysis revealed the interaction of $D D X 3$ with elF4G and PABP proteins under specific stress conditions; DED1 was also observed to bind to elF4G in yeast ${ }^{135}$. Recent evidence suggest that $D H X 29$, $D H X 9$, and $D D X 3$ in mammalians and DED1 in yeast appear to be specific for scanning and unwinding of more complex mRNA structures ${ }^{90,91,96,136}$ whereas the main role of elF4A in elF4F complex appears to be linked to attachment of $43 \mathrm{~S}$ to $\mathrm{mRNA}$ and the scanning for moderately structured mRNAs $90,91,131,134,137$. Other helicases including DDX3 bind to specific structures on $5^{\prime}$-UTR of specific mRNAs and regulate their translation by binding to elF4A or independently like $D b p 5$ helicase. ${ }^{91}$. 
mRNAs having long and highly structured 5'-UTRs requires a different mechanism for efficient translation ${ }^{96}$. This encouraged us to look and investigate more unknown helicases involved in recognition and unwinding of highly structured 5'-UTRs.

The sequence around the AUG start codon is a strong determinant of the translation rate, referred to kozak consensus sequence. If this sequence is not optimal the scanning ribosomes may bypass the first AUG codon and start translation from the second AUG. Such events can be regulated by the presence of a hairpin between the two AUGs. In this case, the balance between the production of the two ORFs, a longer and a shorter one, might be achieved by the formation of an mRNA structure between the two start codons and protein factors that influence the structure ${ }^{73}$. This phenomenon has been observed in plants, animal and yeast cells ${ }^{138}$.

In prokaryotes, structural cis-acting components at 5'-UTR including riboswitches and RNA thermosensors may regulate translation ${ }^{139}$. Riboswitches respond to the binding of metabolites where thermosensors make secondary structures in response to temperature and regulate recruitment of the 305 ribosomal subunit ${ }^{139}$. In general translation initiation region of prokaryotic mRNAs contain an SD sequence. The length of this sequence, its distance to start codon and its involvement in secondary structures also play important roles in the regulation of translation. mRNAs with strong hairpin structures near the start site, generally require stronger SD sequence to efficiently attract the ribosome to the initiation site ${ }^{73}$. Polycistronic mRNAs often carry their own SD sequences upstream of different ORF. Therefore, the translation of each ORF is directly dependent on its corresponding SD sequence. In certain cases, the ribosome attached to the 
upstream ORF is recruited to translate a downstream ORF. In such cases, the presence of a strong secondary structure in the translation region of the downstream ORF often inhibits the recruitment of the ribosomes from the upstream ORF ${ }^{73}$. Certain conditions like low/high temperatures or the presence of certain chemicals can induce specific folding of the mRNAs to inhibit or promote the translation of a specific gene ${ }^{73}$. mRNA folding in bacteria in response to environmental changes can also regulate mRNA stability. Certain repressor proteins can also bind to the upstream regions of the SD sequence and cause the mRNA to fold and prohibit the 30 s from binding to mRNA ${ }^{139}$. Small trans-acting RNAs can also interfere with mRNA structures and regulate prokaryotic translation ${ }^{73,139}$.

\subsection{Focus}

The process of protein synthesis is an essential measure for living cells. In addition to the need for studying translation as an essential process for cell survival, dysfunction in this process has been linked to important human conditions including complex diseases like cancer and diabetes highlighting the need to better understand this process. Although much has been learned over the years, there are a number of processes within translation that require more attention. The list of genes that control translation or influence its efficacy continues to increase.

One of the main objectives of this thesis is to discover and study novel genes that can affect translation in yeast. Specifically, I am interested in studying those genes that affect the translation of structured mRNAs. For this, I employ the yeast gene knockout collection as a tool to screen for those genes that when deleted, expression of a structured mRNA 
reporter is reduced (Figure 1.4). For this purpose, a B-galactosidase reporter gene that carries a series of strong inhibitory structures on it 5'-UTR is used. Gene deletion mutants that were identified in our large scale screening are selected for follow up investigation to better elucidate their involvement in the translation of structured mRNA. In addition, by manipulating the UTRs of a reporter mRNA, I investigated the delivery of this mRNA into yeast mitochondria.

The specific objectives of this thesis are to:

1 Screen the yeast non-essential gene knockout collection for genes that when deleted reduce the expression of a highly structured reporter gene.

2 Investigate the activity of YTA6 and YBRO96C as novel genes that affect the translation of structured mRNAs (Chapter 2).

3 Study the involvement of NAM7, PUS2, and RPL27B in the translation of structured mRNAs (Chapter 3).

4 Examine the ability of the $3^{\prime}$-UTR of yeast OXA1 gene in directing mRNAs into yeast mitochondria (Chapter 4).

5 Design and utilize other structured mRNAs to further screen the yeast nonessential gene knockout collection for additional genes that are required for the translation of structured mRNAs. (Chapter 5). 


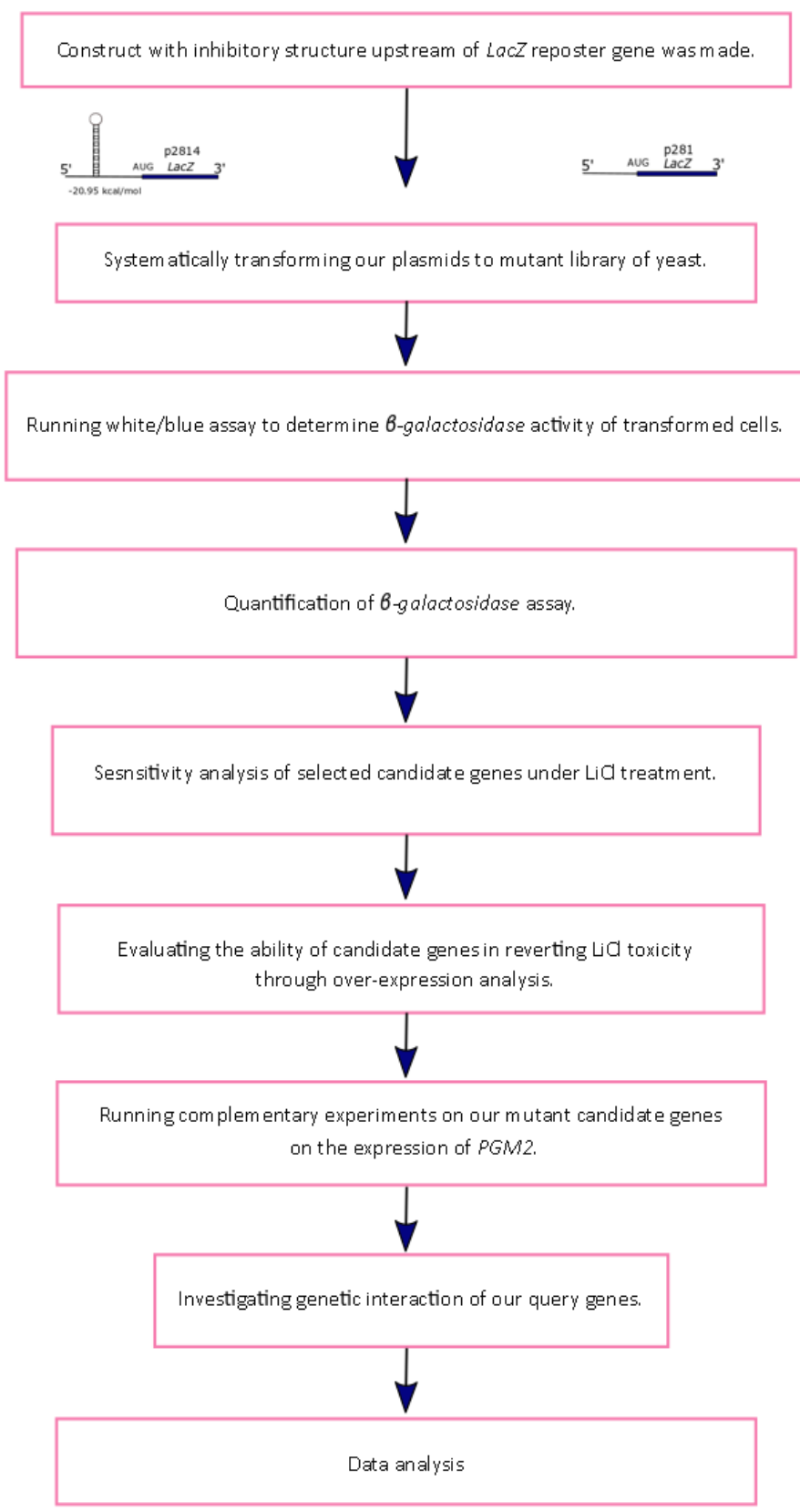

Figure 1.4. Diagram showing the step by step approach in this thesis. A construct with inhibitory structure upstream of $L a c Z$ cassette and a control plasmid were constructed 157. Plasmids were transformed to deletion mutant library to determine in the absence of which candidate genes the cells were unable to produce 6 -galactosidase. 24 candidate genes were identified that did not show translation activity in large-scale lift assay (Blue/White assay). Translation activity was quantified using small scale 6 galactosidase liquid assay to follow up with our query genes we ran sensitivity assay using treatment with $\mathrm{LiCl}$, which has been shown to affect translation initiation linked to TIF2 (elF4A) helicase. It is reported that over-expression of elF4A, the main helicase enzyme linked to translation initiation has been shown to revert $\mathrm{LiCl}$ sensitivity. We repeated this with over-expression of our query genes. Five candidate genes were selected. To further study the involvement of these target genes in the translation of structured mRNAs we ran a series of complementary assays including genetic interaction (GI) analysis. 


\section{Sensitivity of yeast to lithium chloride connects the activity of YTA6 and YPR096C to translation of structured mRNAs}

\subsection{Abstract}

Lithium Chloride (LiCl) toxicity, mode of action and cellular responses have been the subject of active investigations over the past decades. In yeast, LiCl treatment is reported to reduce the activity and alters the expression of $P G M 2$, a gene that encodes a phosphoglucomutase involved in sugar metabolism. Reduced activity of phosphoglucomutase in the presence of galactose causes an accumulation of glucose-1$\mathrm{p}$ leading to a number of phenotypes including growth defect. In the current study, we identify two understudied genes, YTA6 and YPRO96C that when deleted increase cell sensitivity to LiCl treatment in yeast. We further show that YTA6 and YPRO96C exert their activities by influencing PGM2 at the level of translation.

\subsection{Introduction}

Dysregulation of signaling pathways in the brain is thought to be the main cause of bipolar disorder (BD) ${ }^{140}$. LiCl has remained an important treatment option for BD for more than ten decades ${ }^{141,142}$. It has been prescribed to prevent both new depressive and manic episodes and is known to be the only compound to have anti-suicidal effects in BD patients ${ }^{143}$.

When LiCl is used as a therapeutic agent, it is generally accepted that in the short term, it influences Protein Kinase C (PKC) and glycogen synthesis kinase-3 (GSK-3) signal transduction pathways. Long term exposure to LiCl modifies the expression of different 
genes/pathways including $\mathrm{PI} / \mathrm{PKC}$ signaling cascade, leading to alterations in the synaptic function of nerve cells ${ }^{140,144-146}$. Inducing autophagy, oxidative metabolism, apoptosis and affecting translation machinery are other pathways proposed to be influenced by LiCl intake $^{141,145}$. LiCl has also been investigated as a treatment option for Alzheimer's disease which is caused by the aging of the nervous system ${ }^{145,147}$. Although much has been learned about the influence of $\mathrm{LiCl}$, how it affects the cell at the molecular level and the mechanism(s) of its activity, as well as its side effects (secondary effects) require further investigations ${ }^{140,141,147}$.

At the molecular level, the sensitivity of yeast cells to LiCl was previously described by changes in the level of expression and activity for $P G M 2$ that encodes a phosphoglucomutase ${ }^{148,149}$. Phosphoglucomutase is responsible for converting glucose1-phosphate to glucose-6-phosphate. When galactose is used as the carbon source, inhibition of phosphoglucomutase by LiCl results in the accumulation of glucose-1phosphate that in turn causes growth defects ${ }^{150,151}$. In the presence of glucose, LiCl reduces the levels of UDP-glucose and disrupts the associated pathways. It has also been suggested that LiCl may inhibit RNA processing enzymes ${ }^{152,153}$. Also, it is reported that under $\mathrm{LiCl}$ stress, there seems to be a rapid loss of ribosomal protein gene (RBG) premRNAs and a decrease in a number of mature mRNAs in the cytoplasm ${ }^{153}$. In addition, it is possible that LiCl may inhibit the initial steps of the protein synthesis pathway. It is thought that LiCl may disrupt the association of translation initiation factor elF4A RNA helicase to the yeast translation machinery ${ }^{148}$ impairing translation initiation. Deletion of 
TIF2 that codes for the elF4A helicase increased yeast sensitivity to LiCl. Over-expression of elF4A helicase reverted the translational inhibition caused by $\mathrm{LiCl}^{148}$.

In the current study, we observed that the deletion of two yeast genes, YTA6 and YPRO96C increased the sensitivity of yeast cells to LiCl. YTA6 codes for a putative ATPase of the CDC48/PAS1/SEC18 (AAA) family of proteins and YPR096C codes for a protein of unknown function. Neither of the genes was previously linked to cell responses to LiCl. Our followup genetic investigations suggest that the involvement of YTA6 and YPRO96C in yeast LiCl sensitivity seems to be due to their influence on PGM2 translation.

\subsection{Materials and methods}

\subsubsection{Strains, plasmids, gene collections and cell and DNA manipulations}

MATa mating strain Y4741 orf $\Delta:: K a n M A X 4$ his $3 \Delta 1$ leu2 $\Delta 0$ met15 $\Delta 0$ ura3 $\Delta 0$ and MAT $\alpha$ mating strain, Y7092 can1 $\Delta::$ STE2pr-Sp_his5 lyp1 $\Delta$ his3 $\Delta 1$ leu21 $\Delta 0$ ura3 $\Delta 0$ met15 $\Delta 0$ were used. Yeast non-essential gene knockout collections ${ }^{154}$, yeast over-expression plasmid library ${ }^{155}$ and the collection of yeast gene-GFP fusion strains were utilized as before 29,31,33. Yeast gene knockout was performed by PCR transformation using the Lithium Acetate method ${ }^{156}$.

p281 construct carries a LacZ expression cassette under the control of a gal promoter. p281-4 construct carries an insert with a strong hairpin structure (5'GATCCTAGGATCCTAGGATCCTAGG ATCCTAG3') upstream of LacZ cassette ${ }^{157}$. pAG25 plasmid was used as a DNA template for nourseothricin sulfate (NAT) resistance gene marker in PCR reactions for gene knockout experiments. Kanamycin and NAT markers 
were used as selection markers for corresponding deletion mutant strains. All plasmids carried an ampicillin resistance gene which was used as a selection marker in E. coli DH5 $\alpha$, and a URA3 marker gene for selection in yeast.

P416 construct carries a LacZ expression cassette under the transcriptional control of a gal promoter. To generate reporter LacZ mRNAs under the translational control of complex RNA structures, three different fragments were cloned upstream of the LacZ mRNA in p416 construct using $X b a l$ restriction site. In this way three expression constructs were designed as follows: pPGM2 construct contains the 5'-UTR of PGM2 gene (5'TAATAAGAAAAAGATCACCAATCTTTCTCAGTAAAAAAAGAACAAAAGTTAACATAACAT 3'), pTAR construct contains the 5'-UTR of HIV1-tar gene (5'GGGTTCTCTGGTTAGCCAGATC TGAGCCCGGGAGCTCTCTGGCTAGCTAGGGAACCCACTGCTTAAGCCTCAATAAAGCTTGCCTG AGTGCTTCAAGTAGTGTGTGCC $3^{\prime}$ ) and pRTN that contains the $5^{\prime}$-UTR of FOAP-11 gene (5'GGGATTTTTACATCGTCTTGGTAAAGGCGTGTGACCCATAGGTTTTTTAGATCAAACACGTCT TTACAAAGGTGATCTAAGTATCTC $\left.3^{\prime}\right)$.

YP (1\% Yeast extract, $2 \%$ Peptone) or SC (Synthetic Complete) with selective amino acids ( $0.67 \%$ Yeast nitrogen base w/o amino acids, $0.2 \%$ Dropout mix, ) either with $2 \%$ dextrose or $2 \%$ galactose, as a source of carbohydrates, was used as culture medium for yeast and LB (Lysogeny Broth) was used for E. coli cultures. 2\% agar was used for all solid media. Yeast plasmid extraction was performed using yeast plasmid miniprep kit (Omega Biotek $^{\circledast}$ ) and E. coli plasmid extraction was carried out using GeneJET plasmid miniprep kit (Thermofisher ${ }^{\circledR}$ and Bio-Basics ${ }^{\circledR}$ ) according to the manufacturers' instructions. 


\subsubsection{Drug sensitivity analysis}

Yeast cells were grown from independent colonies to saturation for two days at $30^{\circ} \mathrm{C}$ in liquid YPgal. Serial dilutions of cell suspensions were spotted onto solid media with or without $\mathrm{LiCl}$. Sensitivity to the compound was assessed by comparing the number and size of the colonies formed on each plate after 48 hours in comparison with wild type ${ }^{30}$.

For quantification analysis, colony counting was done by taking $100 \mu \mathrm{L}$ of diluted $\left(10^{-4}\right)$ cell cultures from independent colonies, grown for 2 days at $30^{\circ} \mathrm{C}$ in liquid YPgal, and spreading on YPgal plates in the absence and presence of $\mathrm{LiCl}$. The colonies were counted two days after incubation at $30^{\circ} \mathrm{C}$. Each experiment was repeated at least three times. $\mathrm{t}$ test analysis ( $P$-value $\leq 0.05)$ was used to determine statistically significant results.

\subsubsection{Quantitative 6-galactosidase Assay}

To evaluate the activity of LacZ expression cassette in different mutant strains, the quantitative 6 -galactosidase assay was performed using ONPG (O-nitrophenyl- $\alpha$-Dgalactopyranoside) as described ${ }^{32,158}$. Each experiment was repeated at least three times.

\subsubsection{Quantitative real time PCR (qRT-PCR)}

Deletion mutants in PGM2-GFP strain background were grown in YPgal overnight with or without $\mathrm{LiCl}$ treatment. Total RNA was extracted with Qiagen ${ }^{\circledR}$ RNeasy Mini Kit. Complementary DNA (cDNA) was made using iScript Select cDNA Synthesis Kit (Bio-Rad ${ }^{\circledR}$ ) according to the manufacturer's instructions. cDNA was then used as a template for 
quantitative PCR. qPCR was carried out using Bio-Rad ${ }^{\circledR}$ iQ SYBR Green Supermix and the CFX connect real time system (Bio-Rad ${ }^{\circledR}$ ), according to the manufacturer's instructions. PGK1 was used as a constitutive housekeeping gene (internal control).

The procedure was done in three repeats and t-test analysis $(P$-value $\leq 0.05)$ was used to determine statistically significant results. The following primers were used to quantify PGM2 and PGK1 mRNAs, as our positive control in different mutant strains.

PGM2: Forward GGTGACTCCGTCGCAATTAT; Reverse: CGTCGAACAAAGCACAGAAA

PGK1: Forward ATGTCTTTATCTTCAAAGTT; Revers: TTATTTCTTTTCGGATAAGA

\subsubsection{Western blot analysis}

Strains were grown in media treated with and without LiCl. Protein extraction was performed as described by Szymanski ${ }^{159}$. Bicinchoninic acid assay (BCA) was performed to estimate protein concentration as described by the manufacturer (Thermo Fisher ${ }^{\circledR}$ ). Equal amounts of total protein extract $(50 \mu \mathrm{g})$ were loaded onto a $10 \%$ SDS-PAGE gel, run on MiniPROTEAN Tetra cell electrophoresis apparatus system (Bio-Rad ${ }^{\circledR}$ ). Proteins were transferred to a nitrocellulose $0.45 \mu \mathrm{m}$ membrane via a Trans-Blot Semi-Dry Transfer (Bio-Rad ${ }^{\circledR}$ ). Mouse monoclonal anti-GFP antibody (Santa $\mathrm{Cruz}^{\circledR}$ ) was used to detect protein levels of Pgm2p-GFP. Mouse anti-Pgk1 (Santa $\mathrm{Cruz}^{\circledR}$ ) was used to detect Pgk1 protein levels used as internal controls. Immunoblots were visualized with chemiluminescent substrates (Bio-Rad ${ }^{\circledR}$ ) on a Vilber Lourmat gel doc Fusion FX5-XT (Vilber $\left.{ }^{\circledR}\right)$. Densitometry analysis was carried out using the FUSION FX software $\left(\right.$ Vilber $\left.^{\circledR}\right)$. Experiments were repeated at least three times; t-test analysis $(P$-value $\leq 0.05)$ was used to determine statistically significant results. 


\subsubsection{Genetic interaction analysis}

Synthetic genetic analysis was performed in a 384 format as before ${ }^{31,33,51}$. In brief, deletion mutant for query gene in Mat $\alpha$ mating type was crossed to two sets of gene deletion mutants in Mat a mating type. After a few rounds of selection, double gene deletion mutants were selected in Mat a mating type. Colony size measurement was used to compare the fitness of double gene deletion mutants to single deletion strains. Colony size was measured as described before ${ }^{24,54}$. The colony size assay was repeated three times. For Phenotypic Suppression Array (PSA) analysis in SDL, a MATa yeast strain having an over-expression plasmid of our query gene is mated separately with each strain of the entire deletion set. with the library strains containing an empty plasmid was used as our reference for fitness control to address false positives. For phenotypic suppression analysis, the final constructs transformed into deletion library were grown on YPgal compared to the control plasmid. Phenotypic suppression array was performed by growing the transformed cells on YPgal with the sub-inhibitory concentration of $\mathrm{LiCl}$ (3 $\mathrm{mM})$ as a stress condition drug ${ }^{34}$. We investigated the ability of over-expression of our query genes to compensate for the sick phenotype of our deletion sets under the inhibitory concentration of $\mathrm{LiCl}$. If the over-expression of our candidate genes overcome the sensitivity of a yeast deletion strain caused by drug inhibition, we can suggest that a functional connection occurs between these two genes ${ }^{30,160}$. 


\subsubsection{Genetic interaction Data Analysis}

Scoring fitness was done by colony size measurement ${ }^{24,54}$. Those deletions that had $30 \%$ or more reduction in fitness in at least two experiments were considered hits. Based on their biological process and/or molecular function, hits were clustered into groups with enriched GO terms using Gene Ontology Resource http://geneontology.org/ and Genemania database http://genemania.org.

\subsection{Results and Discussion}

\subsubsection{Deletion of YTA6 and YPR096C increases yeast sensitivity to Lithium}

Although the biology of BD has been extensively studied for decades, the molecular basis and the pathophysiology of this disorder is not clearly understood. It is known that DB causes significant neuroanatomical changes affecting frontal subcortical and prefrontal limbic brain regions ${ }^{141}$. However, how these changes occur in a mechanistic and at the molecular level has baffled scientists for years. $\mathrm{LiCl}$ has been used as an effective treatment option for BD for decades. Investigating the $\mathrm{LiCl}$ mode of action and its side effects as well as the molecular responses of a cell to $\mathrm{LiCl}$ may lead to a better understanding of BD's biology.

In mammalian systems, $\mathrm{LiCl}$ inhibits glycogen synthase kinase $3 \beta$. It also affects neurotrophic factors, neurotransmitters, and oxidative metabolism ${ }^{161}$. Numerous studies have also hypothesized alterations in gene expression as a result of $\mathrm{LiCl}^{153}$. In 
yeast PGM2, a key galactose metabolism gene is targeted by $\mathrm{LiCl}$ and over-expression of Translation Initiation Factor TIF2 that codes for elF4A, increases cell resistance to LiCl ${ }^{148}$.

Drug sensitivity of mutant strains to a target chemical is an important tool to investigate how a chemical compound affects the cell at the molecular level and pathways influenced by the drug $31,33,57,162$. While investigating yeast gene deletion mutants that are sensitive to LiCl we identified two gene deletion mutants for YTA6 and YPRO96C that showed increased sensitivity to LiCl. Little is known about the molecular activity of these two genes and the cellular process in which they participate making them interesting targets to study. YTA6 codes for a putative ATPase and YPRO96C is an uncharacterized ORF.

In the spot test assay indicated in Fig 2.1 yta6 $\Delta$ and ypr096c $\Delta$ show growth reduction in the presence of $\mathrm{LiCl}(10 \mathrm{mM} \mathrm{LiCl})$ suggesting increased sensitivity of yeast strains when either of these two genes is deleted. tif $2 \Delta$ was used as a positive control. To confirm that the observed phenotypic deficiency (sensitivity to LiCl) was due to the deletion of the target genes (YTA6 and YPRO96C) and not an effect of an unintended mutation in a secondary site, we re-introduced the ORFs back into their corresponding mutant strains. We observed that the re-introduction of the target genes recovered the fitness of the cells (Fig 2.1). To confirm the results obtained by the spot test assay we perform colony count measurement analysis, which represents a more quantitative approach. In this method, the decreased percentage of colonies is calculated by dividing the number of colonies in media in the presence of the LiCl to the number of colonies in control media and normalized to Wild Type (WT). Indicated in Fig 2 deletion of YTA6, YPRO96C or TIF2 
show reduced colony formation in the presence of LiCl. Reintroduction of the deleted genes into their corresponding gene deletion strains suppressed cell sensitivities to LiCl caused by gene deletions.

$\mathrm{LiCl}$ reduces the activity of phosphoglucomutase enzyme leading to the accumulation of galactose-1-phosphate, a toxic intermediate in galactose metabolism. To investigate the influence of YTA6 and YPRO96C on LiCl toxicity through galactose metabolism, we generated double gene deletions for YTA6 or YPRO96C with the GAL1 gene. The deletion of GAL1 gene relieved the sensitivity of gene deletion mutants for YTA6 or YPRO96C to LiCl (Figure 2.1). Also, the sensitivity of deletions strains for YTA6 or YPRO96C to LiCl diminished when glucose was used as a carbon source further connecting the observed LiCl sensitivity for YTA6 and YPRO96C deletion strains to galactose metabolism.

A)

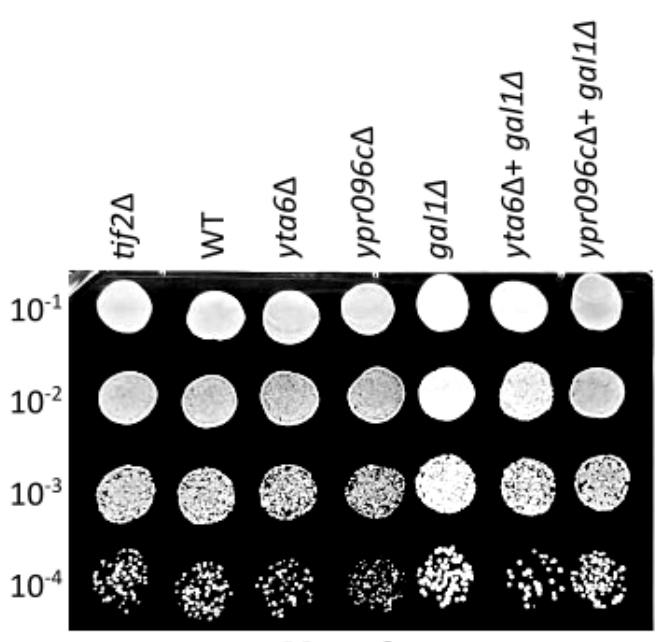

Ypgal
B)

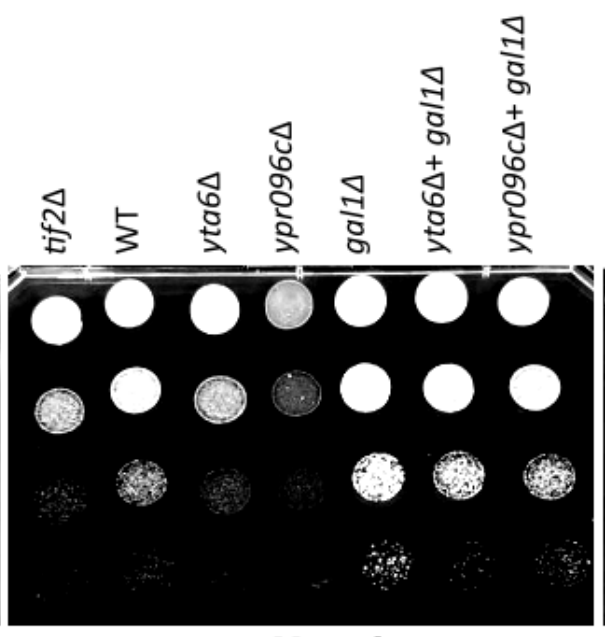

Ypgal

10 mM LiCl
C)

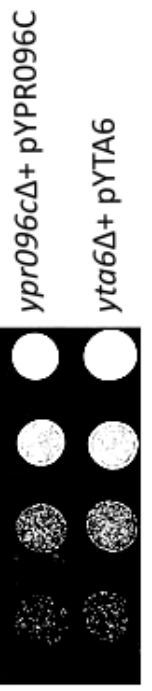

Ypgal

10 mM LiCl 
D)

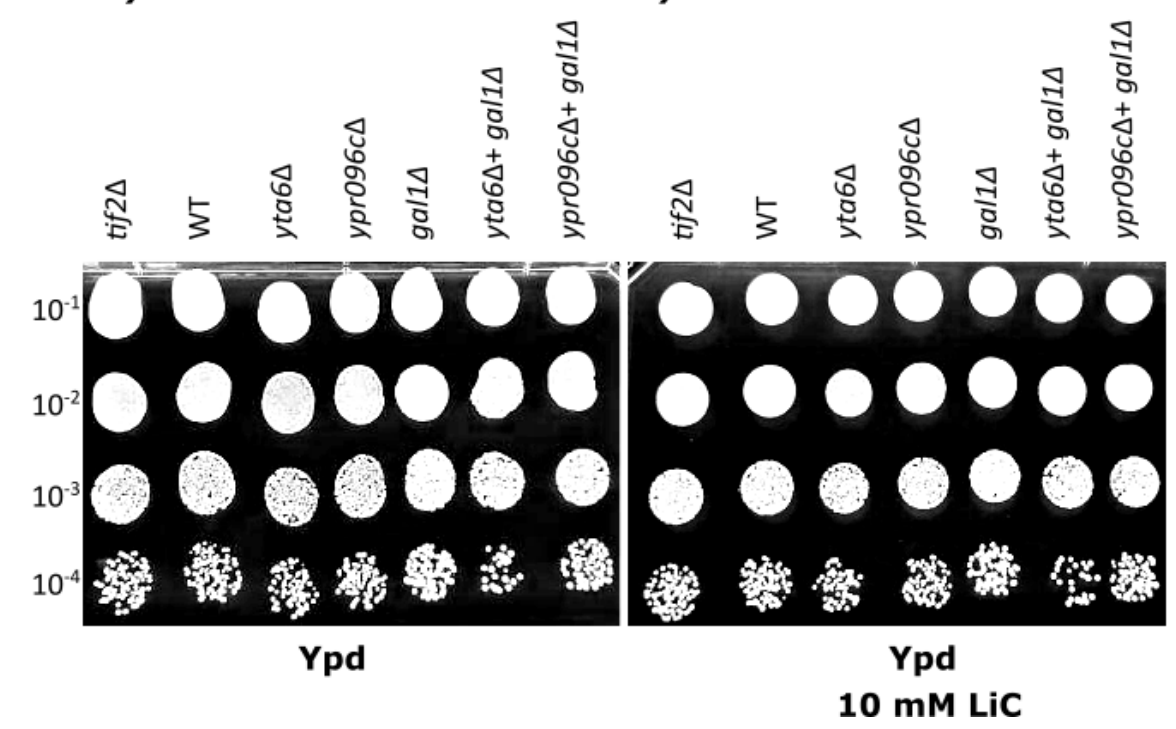

Figure 2.1. Drug sensitivity analysis for different yeast strains using spot test analysis. In $(A)$ and $(B)$ yeast cells were serially diluted as indicated $\left(10^{-1}\right.$ to $\left.10^{-4}\right)$ and spotted on

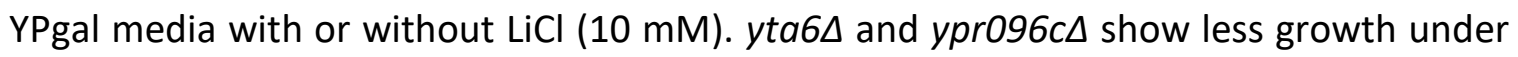
$\mathrm{LiCl}$ treatment. Double deletion for GAL1 with YTA6 or YPR096C suppressed the observed sensitivity of single-gene deletions for YTA6 or YPRO96C. The deletion of TIF2 was used as a positive control. In (C) over-expression of the target gene in their corresponding deletion mutants reverted cell sensitivity to $\mathrm{LiCl}(10 \mathrm{mM})$. In (D) and (E) no $\mathrm{LiCl}$ toxicity was observed in media containing glucose as another carbon source. 


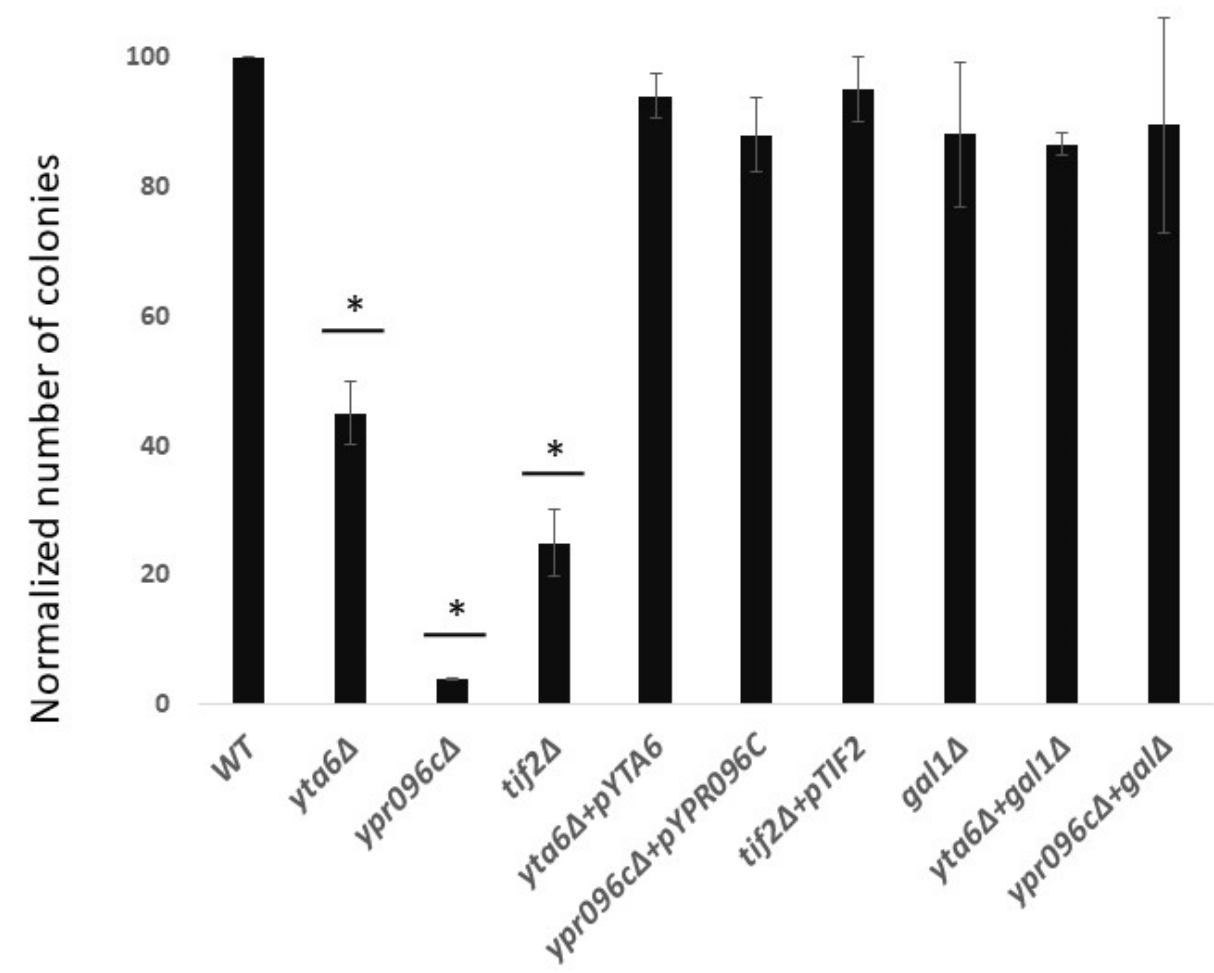

Figure 2.2. Quantitative analysis of drug sensitivity for different yeast strains. The average number of colonies formed for different yeast strains in the presence of $\mathrm{LiCl}$ (10 $\mathrm{mM}$ ) was normalized to that for WT strain. Double deletion for GAL1 with YTA6 or YPRO96C suppressed the observed sensitivity of single-gene deletions for YTA6 or YPRO96C. Data represent the average from three independent experiments and error bars represent standard deviation. * represent statistically significant results compared to the WT $(P$-value $\leq 0.05)$.

\subsubsection{YTA6 and YPR096C regulate the expression of PGM2 at the level of translation.}

PGM2 has been identified as a target of $\mathrm{LiCl}$ in yeast cells and its expression has been reported to change in the presence of $\mathrm{LiCl}^{149}$. Next, we investigated the ability of YTA6 and YPRO96C to change PGM2 expression both at the levels of protein abundance (Figure 
2.3A) and transcription (Figure 2.3B). This was done using western blot analysis in a strain where Pgm2p was tagged with a GFP gene. In the absence of $\mathrm{LiCl}$, we observed no notable alteration in the Pgm2p levels when either YTA6 or YPRO96C were deleted. However, when cells were challenged with $10 \mathrm{mM} \mathrm{LiCl}$, the deletion of either YTA6 or YPRO96C reduced the protein content of Pgm2p.

To investigate the possible effect of YTA6 and YPR096C on PGM2 transcription, we used qRT-PCR analysis to measure the content of PGM2 mRNA when YTA6 and YPRO96C were deleted. Indicated in Figure 2.3B, the deletion of YTA6 and YPRO96C did not noticeably change the content of PGM2 mRNA when cells were treated with $\mathrm{LiCl}$. This suggests that YTA6 and YPRO96C are unlikely to alter PGM2 expression at the transcription level. Together these observations connect the activities of YTA6 and YPRO96C to the expression of Pgm2p at the protein level. This is in agreement with a previous observation by Sofola-Adesakin et al. that in Drosophila melanogaster LiCl impaired gene expression at the protein synthesis level and not the mRNA level ${ }^{145}$. 


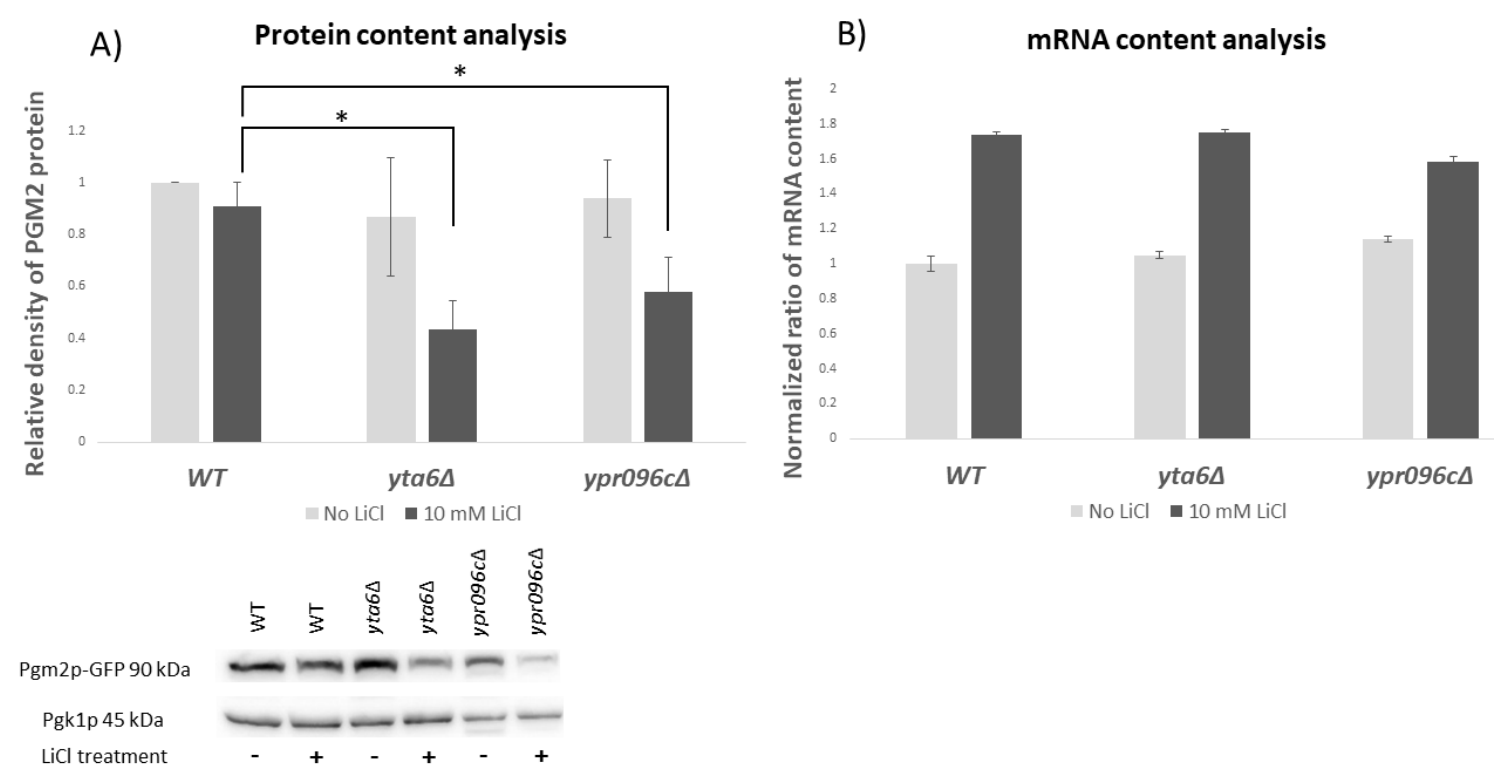

Figure 2.3. Protein and mRNA content analysis (A) Protein content analysis of Pgm2pGFP protein in deletion of yeast strains for $y$ ta6 $\Delta$ and ypr096c $\Delta$. Western blot analysis was used to measure the protein content for Pgm2p-GFP protein in the absence or presence of $\mathrm{LiCl}(10 \mathrm{mM})$ and related to WT. Pgk1p was used as a housekeeping gene and the values are normalized to that. The inset represents a typical blot (B) mRNA content analysis of PGM2 in yta6 $\triangle$ and $y p r 096 c \Delta$. qRT-PCR was used to evaluate the content of PGM2 mRNA in yeast gene deletion mutants related to WT strain and normalized to PGK1 mRNA levels in the absence or presence of $\mathrm{LiCl}(10 \mathrm{mM})$. Each experiment was repeated at least three times $(n \geq 3)$. Error bars represent standard deviation. * represent statistically significant results compared to the value in the corresponding WT. t-test analysis $(P$-value $\leq 0.05)$ was used to compare differences.

\subsubsection{Translation of 6-galactosidase reporter mRNA with a hairpin structure is altered by YTA6 and YPRO96C}

The $5^{\prime}$-UTR of PGM2 mRNA is predicted to contain a highly structured region ${ }^{163}$ (Appendix

Figure 8.1). This knowledge along with the observation that YTA6 and YPRO96C appear to impact PGM2 expression at the protein level prompted us to investigate the influence of YTA6 and YPRO96C on the translation of other structured mRNAs. First, we placed the 5'- 
UTR of PGM2 mRNA in front of a LacZ reporter gene in a p416 expression construct ${ }^{116}$. Indicated in Figure 2.4 ( $\mathrm{A}$ and $\mathrm{B}$ ), when YTA6 and YPRO96C were deleted the activity of Bgalactosidase was reduced for the reporter gene that contained $5^{\prime}$-UTR of PGM2 mRNA and not a control mRNA without the 5'-UTR of PGM2. The deletion of TIF2 was used as a positive control. Next, we utilized an expression cassette, p281-4 with a strong hairpin structure in front of a LacZ reporter gene ${ }^{157}$. A second construct, p281 without the hairpin structure was used as a control. Illustrated in Figure 2.4 (C and D) it was observed that when YTA6 and YPRO96C were deleted the activity of B-galactosidase was reduced for the reporter gene that contained a hairpin structure. When the hairpin was absent, the activity of B-galactosidase was independent of YTA6 and YPRO96C. Together these data show that the deletion of YTA6 and YPRO96C seem to reduce the translation of structured reporter mRNAs. 

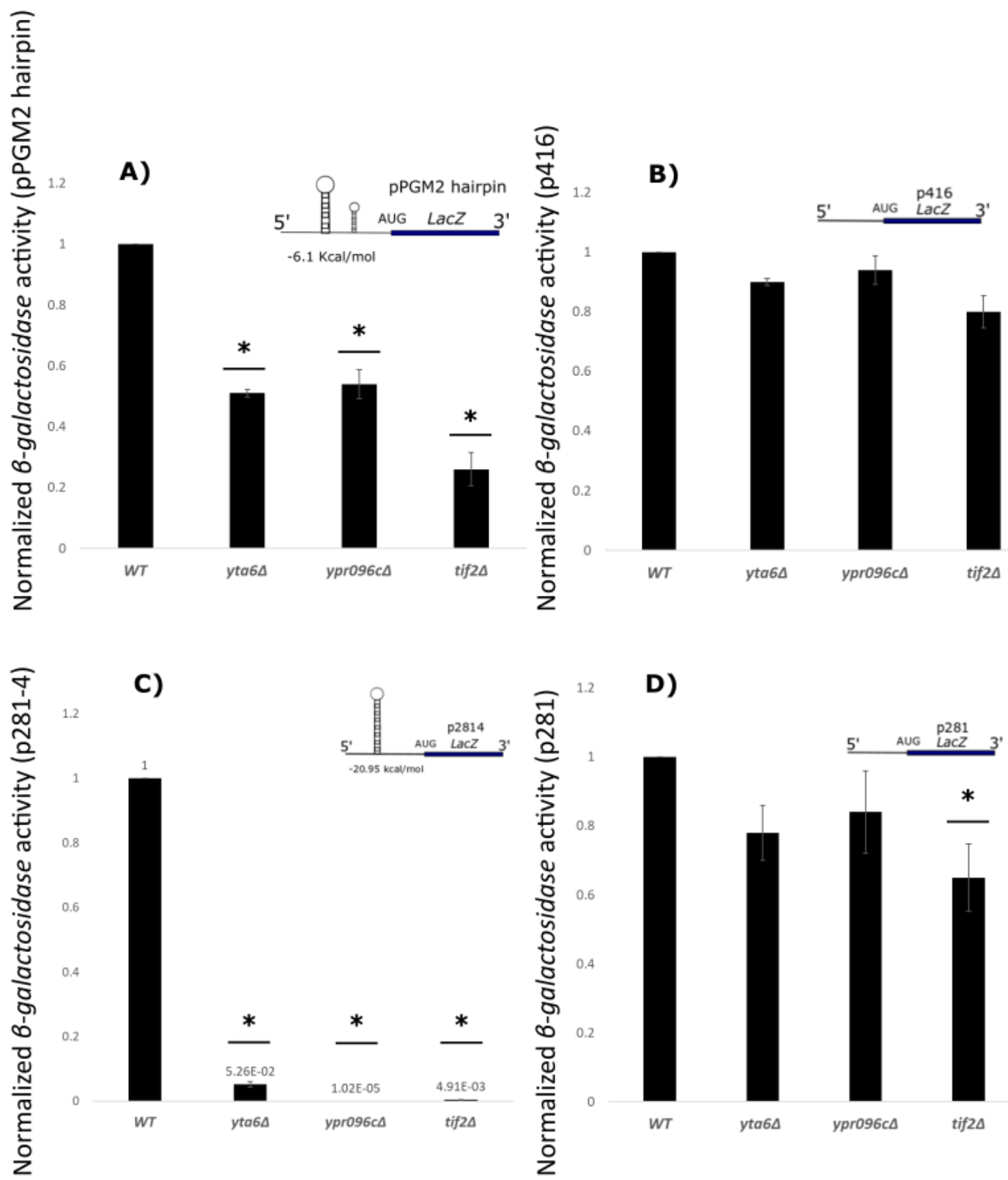

Figure 2.4. 6-galactosidase expression analysis in different yeast strains. Activities from 6-galactosidase mRNAs that carry 5'-UTR of PGM2 mRNA (pPGM2) (A) or a strong hairpin structure (p281-4) (C) upstream of a LacZ reporter were reduced in yta6 6 and ypr096c $\Delta$ strains; tif $2 \Delta$ was used as a positive control. Strains carrying low complexity regions upstream of $L a c Z$ reporters p416 (B) and p281 (D) did not show as significant reductions in B-galactosidase activity. Values are normalized to that for WT. Each experiment was repeated at least three times and error bars represent standard deviation. * represent statistically significant results $(P$-value $\leq 0.05)$. Insets represent schematic representation for the structure of the reporter mRNAs. 
Next, we investigated the influence of YTA6 and YPR096C on other structured mRNAs. For this, we designed two additional B-galactosidase mRNA reporters each carrying different complex RNA structures. pTAR carries the 5'-UTR of the HIV1-tar gene. This region contains a strong hairpin loop involved in modulating expression ${ }^{102}$. pRTN carries the $5^{\prime}-$ UTR of the FOAP-11 gene that contains a highly structured region ${ }^{164}$. Indicated in Figure 2.5, deletion strains for YTA6 and YPRO96C had a highly reduced level of B-galactosidase expression.
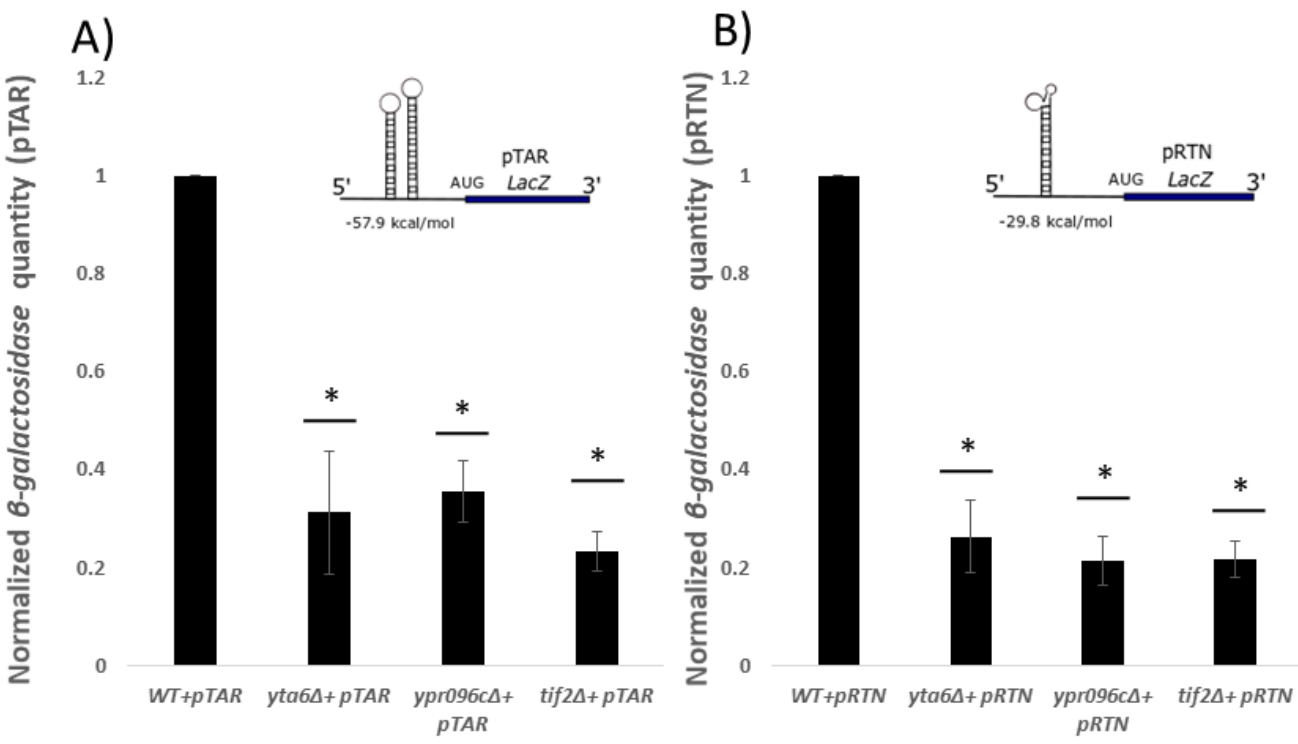

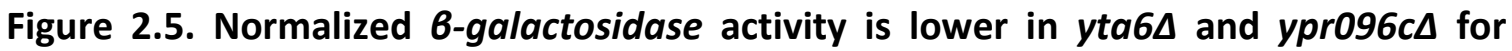
structured mRNAs. pTAR (A) and pRTN (B) constructs contain the highly structured 5 'UTR of HIV1-tar and FOAP-11 genes, respectively, in front of the b-galactosidase reporter mRNA. Values are normalized to that for WT. Each experiment was repeated at least three times and error bars represent standard deviation. * represent statistically significant results $(P$-value $\leq 0.05)$. 


\subsubsection{Genetic interaction analysis further connects the activity of YTA6 and YPR096C to protein biosynthesis}

Genetic interaction (GI) analysis is based on the assumption that parallel compensating cellular pathways give the cell its plasticity and tolerance against random deleterious mutations ${ }^{24}$. In this way, deletion of individual genes that can functionally compensate for each other has little or no phenotypic consequences. However, when both genes are deleted, an unexpected phenotype can emerge which can often be detected by a decrease in cell fitness or even cell death. In this case, the two genes are said to be forming a negative genetic interaction ( $\mathrm{nGI}$ ). An $\mathrm{nGI}$ can reveal the involvement of genes in compensating parallel pathways. $\mathrm{nGI}$ analysis has been used in various investigations to study gene function ${ }^{29,31,34}$. Systematic analysis of Gls in yeast is made possible by its two mating types. A target gene deletion in $\alpha$-mating type (MAT alpha) is crossed with an array of single-gene deletion in a-mating type (MAT a) background and after a few rounds of selection double gene knockouts are obtained ${ }^{51}$. Colony size measurement is often used to determine the fitness of double gene knockouts ${ }^{165}$. To this end, we generated a set of double gene deletions mutants for our two query genes with 768 deletion mutants for genes involved in gene expression. This array was termed gene expression array. Due to inherent bias associated with such enriched subsets, a second set of double gene deletions were made for our query genes with 384 random gene deletions, termed random array, and was used as a control.

YTA6 formed $7 \mathrm{nGls}$ with different genes. The list of interactors includes YPLO79W that encodes for large ribosomal subunit protein 21B and YPLO9OC that codes for small 
ribosomal subunit 6A. YPRO96C interacted with 8 genes including YORO91W that codes for a protein associated with translating ribosomes and YORO78W that codes for a protein involved in small ribosomal subunit biogenesis. The low number of $\mathrm{nGls}$ observed for both YTA6 and YPRO96C makes it difficult to draw a statistically meaningful enrichment for the interacting genes. As a result, formulating function(s) for YTA6 and YPRO96C on the basis of the observed interactions is not feasible.

In addition, we investigated the conditional nGls for the two target genes. Conditional Gls represent an interesting form of gene association. They represent a further insight into the function of genes under a specific condition. The activities of many genes are known to be condition dependent. For example, the expression of many DNA repair genes are regulated in response to DNA damage ${ }^{28,166}$. To this end, we investigated conditional nGls for YTA6 and YPRO96C in the presence of a mild concentration of LiCl (3 mM). Illustrated in Figure 2.6 YTA6 formed a total of 14 conditional nGls. On the basis of their functions and cellular processes in which they participate, these genes can be divided into different categories. Of note, the category of genes involved in protein biosynthesis was the only significantly enriched category $(P=1.6 e-4)$. Within this category, we find 7 genes including, RPL2B that encodes large ribosomal subunit protein 2B and YDR159W that codes for a protein required for biogenesis of small ribosomal subunit. YPRO96C formed 13 conditional $n G l s, 6$ of which belonged to the category of protein biosynthesis $(P=6.6 \mathrm{e}-$ 4). The genes in this category include YDLO81C that codes for ribosomal stalk protein P1 alpha and YER153C that codes for a mitochondrial translation activator. The conditional nGls observed here suggest a functional role for YTA6 and YPRO96C in protein 
biosynthesis when cells are challenged with $\mathrm{LiCl}$.

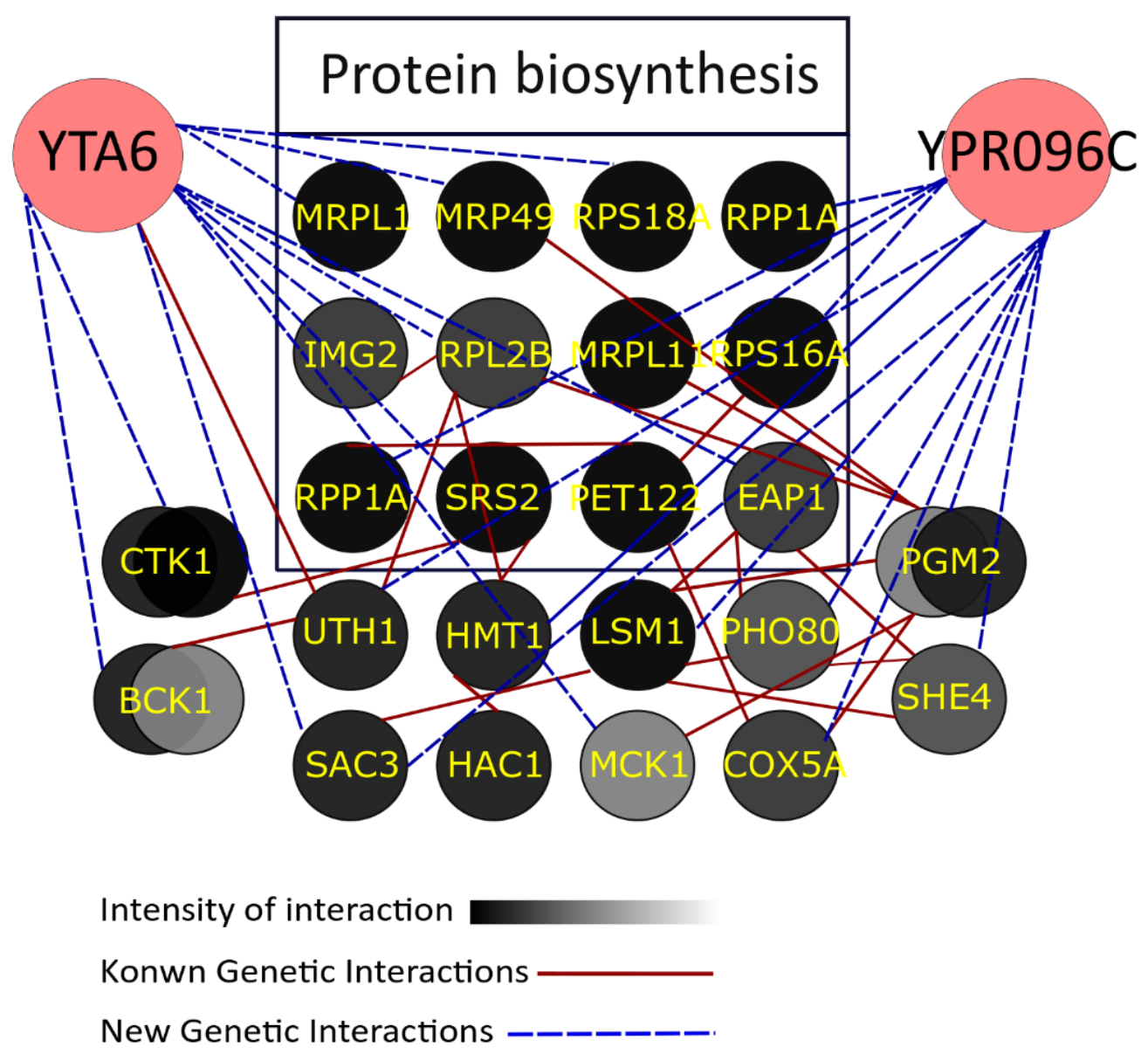

Figure 2.6. Conditional nGIs for YTA6 and YPR096C under $3 \mathrm{mM}$ concentration of LiCl. Our data shows a cluster of interactors involved in the protein biosynthesis pathway for YTA6 ( $\mathrm{P}=1.6 \mathrm{e}-4)$ and YPRO96C ( $\mathrm{P}=6.6 \mathrm{e}-4)$. CTK1, HAC1, BCK1, MRPL1, and PGM2 are mutual hits shared between YTA6 and YPRO96C. Circles represent genes, dashed lines represent $\mathrm{nGls}$ identified in this study and solid lines represent previously reported interactions in the literature.

PSA analysis focuses on another form of Gls, where a specific phenotype associated with a gene deletion mutant is suppressed by the over-expression of the second gene $7,167,168$. This type of GI generally indicates a close functional association where the activity of an 
over-expressed gene compensates for the absence of the others. To this end, we subjected the gene expression array (described above) to $10 \mathrm{mM}$ of $\mathrm{LiCl}$. In this concentration, a number of strains showed sensitivity. We then attempted to reverse the observed sensitivities by over-expression of either YTA6 or YPRO96C in these mutants. Interestingly over-expression of either YTA6 or YPRO96C compensated for the sensitivity of the same two gene deletions, bck1 $\Delta$ and eap1 1 , to LiCl (Figure 2.7). We confirmed our PSA data using spot test drug sensitivity analysis (Figure 2.7). We observed that sensitivity of $b c k 1 \Delta$ and eap1 1 to $10 \mathrm{mM} \mathrm{LiCl}$ was relieved by introducing $p Y T A 6$ and $p Y P R 096 C$ overexpression plasmids into deletion mutant strains (Figure 2.7). The fact that YTA6 and YPRO96C compensated the same two gene deletions, further connects their activities together in the context of $\mathrm{LiCl}$ sensitivity. Bck1 is reported to function in cell wall integrity pathway and deadenylation of mRNAs and Eap1 is an elF4E-associated protein and accelerates the decapping of mRNAs. They have both been implicated in the regulation of alternative translation initiation via Dhh1p, a helicase protein ${ }^{169-172 . ~ D h h 1 p ~ i s ~ a ~}$ member of the DEAD-box family of RNA helicases capable of unwinding strong secondary structures. It functions in mRNA decapping and translational repression among other processes ${ }^{7,173}$. A proposed functional association for both YTA6 and YPRO96C to the regulation of translation via Dhh1 merits further investigations. 


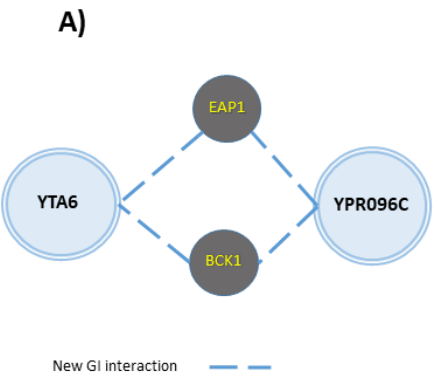

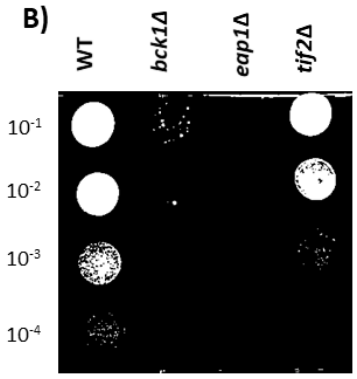

Ypgal+ LiCl (10mM)

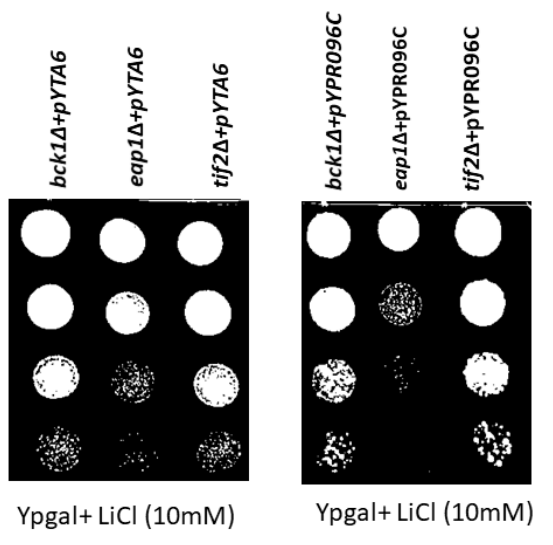

Figure 2.7. Over-expression of YTA6 and YPR096C compensate for the sensitivity of eap1 1 and bck1 10 to $10 \mathrm{mM} \mathrm{LiCl}$. (A) $B C K 1$ and EAP1 are known to be involved in translation initiation via $\mathrm{DHH1}$ through previously reported genetic. New genetic interactions (PSA-based) identified in this study are shown with dashed lines. (B) Spot test

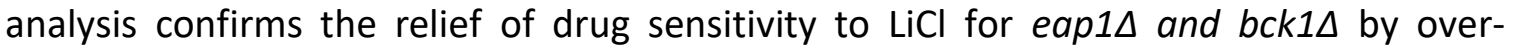
expression of YTA6 and YPRO96C. 


\section{Lithium chloride toxicity is connected to the regulation of gene expression in yeast.}

\subsection{Abstract}

For decades, lithium chloride ( $\mathrm{LiCl}$ ) has been used as a treatment option for those living with bipolar disorder (BD). As a result, many studies have been conducted to examine its mode of action, toxicity, and downstream cellular responses. We know that $\mathrm{LiCl}$ is able to affect cell signaling and signaling transduction pathways through Protein Kinase $C$ and Glycogen Synthase Kinase-3 which are considered to be important in regulating gene expression at the translational level. However, additional downstream effects require further investigation, especially in the translation pathway. In yeast, LiCl treatment affects the expression, and thus the activity, of $P G M 2$, a phosphoglucomutase involved in sugar metabolism. However, it is not fully understood how $\mathrm{LiCl}$ affects gene expression in this matter. In this study, we identified three understudied genes, NAM7, PUS2, and RPL27B which increase $\mathrm{LiCl}$ sensitivity when deleted. We further demonstrate that NAM7, PUS2, and $R P L 27 B$ are involved with the expression of PGM2.

\subsection{Introduction}

$\mathrm{BD}$ is known to be associated with dysregulated signaling pathways. $\mathrm{LiCl}$ has been a key treatment option for those living with BD demonstrating neuroprotective effects for more than six decades ${ }^{174,175}$. It has been used to prevent both depressive and manic episodes and is one of the only compounds available to have anti-suicidal effects in BD patients ${ }^{143}$. 
It has both pro- and anti-inflammatory effects, controlling the increase of inflammatory cytokines in psychiatric disorders ${ }^{176}$.

In simplest terms, LiCl affects both the Protein Kinase C (PKC) and Glycogen Synthase Kinase-3 (GSK-3) signal transduction pathways, which are involved in translation, neurodevelopment, and neuronal plasticity ${ }^{174}$. It is proposed to induce proliferation of neural stem cells through Wnt signaling pathway ${ }^{177}$. Other pathways including cell proliferation, apoptosis, cell fate, and translation machinery are also known to be affected by LiCl $141,145,177,178$.

Since its range of activities is so diverse, $\mathrm{LiCl}$ has been studied as a potential treatment for Alzheimer's disease ${ }^{145,147}$. Alzheimer's disease is well-known for its characteristic features of increased cell death in the dopaminergic regions of the brain due to increased plaques and tangles. In a study conducted by Yan ${ }^{174}$ it was found that the inhibition of GSK-3 $\beta$ through LiCl treatments also had positive implications for adolescents facing the impact of methamphetamine exposure with the potential to prevent long-term behavioral alterations ${ }^{174}$. The efficacy of $\mathrm{LiCl}$ as a treatment for Alzheimer's disease appears to be linked to LiCl's ability to decrease levels of amyloid $\beta$ and decrease phosphorylation of the tau protein. Both of these effects directly target symptoms observed in patients with Alzheimer's disease ${ }^{179}$. LiCl has also been investigated as a treatment option for Parkinson's disease and Huntington's disease ${ }^{145,147}$.

Although much has been learned about the influence of $\mathrm{LiCl}$, it is still unclear how it affects the cell at the molecular level. The effects of $\mathrm{LiCl}$ on gene expression and its secondary 
effects are also not well-known. It's been observed that after long term treatment of BD patients with $\mathrm{LiCl}$, it affects gene expression of genes involved in the $\mathrm{PI} / \mathrm{PKC}$ signaling cascade, consequently causing disruption in the function of nerve cells ${ }^{140,144-146}$, renal cells and liver cell damages ${ }^{175}$ which requires further investigation of the mechanism(s) of its activity, as well as secondary effects ${ }^{140,141,147}$.

Yeast is particularly sensitive to $\mathrm{LiCl}$ treatment because it alters the expression and activity of $P G M 2$, a phosphoglucomutase that mediates the entry of galactose into glycolysis 148,150. PGM2 also converts glucose-1-P to glucose-6-P, and if inhibited, it leads to the accumulation of glucose-1-P causing toxicity in yeast cells ${ }^{151}$. When $\mathrm{LiCl}$ is added to galactose media, yeast cell growth is severely reduced due to impaired glycolysis. $\mathrm{LiCl}$ also reduces the levels of UDP-glucose and disrupts associated pathways in the presence of glucose. Other studies have also suggested that $\mathrm{LiCl}$ might inhibit RNA processing enzymes and rapidly reduce the expression of ribosomal protein genes (RBG) ${ }^{152,153}$. This results in a decreased number of mature mRNAs in the cytoplasm ${ }^{153}$ suggesting inhibition at the translational level.

Studies are also being done to see if $\mathrm{LiCl}$ inhibits the initial steps of protein synthesis. One study has demonstrated that $\mathrm{LiCl}$ may be able to disrupt the association of translation initiation factor elF4A, an RNA helicase, to the rest of the translational machinery, impairing translation initiation ${ }^{148}$. However, this was not observed in yeasts growing on glucose media as a carbon source. 
NAM7 is an important gene involved in efficient termination of translation, especially in nonsense-mediated mRNA decay. This gene codes for an RNA helicase that binds to the small ribosomal subunit. PUS2 is involved in tRNA modification in the mitochondria and also pseudouridylation of some nuclear mRNAs. And RPL27B encodes for the large ribosomal subunit. In follow-up genetic studies, we suggest that the deletion of NAM7, PUS2, and RPL27B increases yeast sensitivity to $\mathrm{LiCl}$ through the expression of PGM2. In this study, we demonstrate that the deletion of yeast genes NAM7, PUS2, and RPL27B increases the sensitivity of yeast to $\mathrm{LiCl}$. These genes have never been studied to cell responses linked to $\mathrm{LiCl}$ before.

\subsection{Materials and Methods}

\subsubsection{Strains, plasmids, gene collections and cell and DNA manipulations}

MATa mating strain Y4741 orf $\Delta:: K a n M A X 4$ his $3 \Delta 1$ leu2 $\Delta 0$ met15 $\Delta 0$ ura3 $\Delta 0$ and MAT $\alpha$ mating strain, Y7092 can $1 \Delta::$ STE2pr-Sp_his5 lyp1 $\Delta$ his $3 \Delta 1$ leu21 $\Delta 0$ ura3 $\Delta 0$ met $15 \Delta 0$ were used. Deleted mutant strains were obtained from yeast non-essential gene knockout mutant library ${ }^{50}$. This library was also used for Synthetic Genetic Analysis to make double mutants, overexpression plasmid of candidate genes were extracted from yeast overexpression collection ${ }^{155}$ and yeast PGM2-GFP fusion strain was purchased from Yeast GFP Clone Collection from Thermofisher ${ }^{\circledR}$ which was used for qPCR and western analysis purposes as described before ${ }^{29,31,33}$. Yeast gene deletions in MAT $\alpha$ strain were performed by PCR transformation and homologous recombination through the Lithium Acetate method as described in previous experiments ${ }^{156}$. 
p281 construct, carrying a LacZ expression cassette under the control of a gal promoter, was used as our control plasmid. p281-4 with a strong hairpin (5' GATCCTAGGATCCTAGG ATCCTAGG ATCCTAG 3'), inserted upstream of LacZ expression cassette was used as our investigating construct ${ }^{157}$. pAG25 plasmid was used as a DNA template in PCR reactions to amplify nourseothricin sulfate (NAT) resistance gene marker, to identify successful transformation in gene knockout experiments. All plasmids carried an ampicillin resistance gene which was used as a selection marker in E. coli DH5 $\alpha$, and a URA3 marker gene for selection in yeast. Kanamycin and NAT drug was used as selectable markers for gene knock out in Mat " $a$ " and Mat " $\alpha$ " respectively.

p416 construct carries a LacZ expression cassette under the transcriptional control of a gal promoter which was used as our plasmid control in translation activity assays. To generate reporter LacZ mRNAs under the translational control of complex RNA structures, three different fragments were cloned upstream of the LacZ mRNA in p416 construct using Xbal restriction site. Using this method, three expression constructs were designed as follows: pTAR construct which contains the 5'-UTR of HIV1-tar gene (5’GGGTTCTCTGGTTAGCCAGATCTGAGCCCGGGAGCTCTCTGGCTAGCTAGGGAACCCACTGC TTAAGCCTCAATAAAGCTTGCCTTGAGTGCTTCAAGTAGTGTGTGCC 3') and pRTN which contains the $5^{\prime}$-UTR of FOAP-11 gene (5'GGGATTTTTACATCGTCTTGGTAAAGGCGTGTGAC CCATAGGTTTTTTAGATCAAACACG TCTTTACA AAGGTGATCTAAGTATCTC 3’).

YP (1\% Yeast extract, 2\% Peptone) or SC (Synthetic Complete) with selective amino acids ( $0.67 \%$ Yeast nitrogen base w/o amino acids, $0.2 \%$ Dropout mix) either with $2 \%$ dextrose or $2 \%$ galactose, as a carbon source, were used as culture medium for yeast and LB 
(Lysogeny Broth) was used for E. coli cultures. 2\% agar was used for all solid media. Yeast plasmid extraction was performed using yeast plasmid miniprep kit (Omega Biotek ${ }^{\circledR}$ ) and E. coli plasmid extraction was carried out using GeneJET plasmid miniprep kit (Thermofisher ${ }^{\circledR}$ and Bio-Basics ${ }^{\circledR}$ ) according to the manufacturers' instructions.

\subsubsection{Drug sensitivity analyses}

To over saturate yeast cells for spot test analysis, cultures were grown in liquid medium for two days at $30^{\circ} \mathrm{C}$ shaker incubator. Following incubation, four serial dilutions $\left(10^{-1}\right.$ to $10^{-4}$ ) of the cell cultures were spotted onto solid media with or without LiCl treatment. After 48 hours, the sensitivity of was determined based on their colony size and number 30.

For drug sensitivity colony count assays, $100 \mu \mathrm{L}$ of diluted $\left(10^{-4}\right)$ oversaturated cell culture, as described above, was spread on YPgal plates in the absence and presence of LiCl. Plates were then analyzed based on colony numbers after incubation at $30^{\circ} \mathrm{C}$ incubator for two days. Each experiment was repeated at least three times. $t$-test analysis ( $p$-value $\leq 0.05$ ) was used to determine statistically significant results from WT control.

\subsubsection{Quantitative 6-galactosidase Assay}

This assay was used to evaluate the activity of $L a c Z$ cassette in different mutant strains as a reference for translation activity. The quantitative 6 -galactosidase assay was performed using ONPG (O-nitrophenyl- $\alpha$-D-galactopyranoside) as described by Krogan et al., ${ }^{32}$. Each 
experiment was repeated at least three times.

\subsubsection{Quantitative real time PCR (qRT-PCR)}

To determine the mRNA level of PGM2 in different deletion mutants, PGM2-GFP yeast strain was grown in YPgal overnight with or without LiCl treatment. Total RNA was extracted with Qiagen ${ }^{\circledR}$ RNeasy Mini Kit. Complementary DNA (cDNA) was made using iScript Select cDNA Synthesis Kit $\left(\right.$ Bio-Rad $\left.^{\circledR}\right)$ according to the manufacturer's instructions. cDNA was then used as a template for quantitative PCR. qPCR was carried out using Bio$\operatorname{Rad}^{\circledR}$ iQ SYBR Green Supermix and the CFX connect real time system (Bio-Rad ${ }^{\circledR}$ ), $\operatorname{according}^{-1}$ to the manufacturers' instructions. PGK1 was used as a constitutive housekeeping gene (internal control).

The procedure was done in three repeats and t-test analysis ( $P$-value $\leq 0.05)$ was used to determine statistically significant results. The following primers were used to quantify PGM2 and PGK1 mRNAs, as our positive control in different mutant strains.

PGM2: Forward GGTGACTCCGTCGCAATTAT; Reverse: CGTCGAACAAAGCACAGAAA PGK1: Forward ATGTCTTTATCTTCAAAGTT; Revers: TTATTTCTTTTCGGATAAGA

\subsubsection{Western blot analysis}

Deleted mutant strains with GFP-tagged PGM2 were grown in media treated with and without $\mathrm{LiCl}$ to investigate protein levels of PGM2 in different conditions. Protein extraction was performed as described by Szymanski et al., ${ }^{159}$. Bicinchoninic acid assay (BCA) was performed to estimate protein concentration as described by the manufacturer 
(Thermo Fisher $\left.{ }^{\circledR}\right)$. Equal amounts of total protein extract $(50 \mu \mathrm{g})$ were loaded onto a $10 \%$ SDS-PAGE gel, run on Mini-PROTEAN Tetra cell electrophoresis apparatus system (Bio$\left.\operatorname{Rad}^{\circledast}\right)$. Proteins were transferred to a nitrocellulose $0.45 \mu \mathrm{m}$ membrane via a Trans-Blot Semi-Dry Transfer (Bio-Rad ${ }^{\varpi}$ ). Mouse monoclonal anti-GFP antibody (Santa $\mathrm{Cruz}^{\circledR}$ ) was used to detect protein levels of Pgm2p-GFP. Mouse anti-Pgk1 (Santa Cruz ${ }^{\circledR}$ ) was used to detect Pgk1p levels, which was used as an internal control. Immunoblots were visualized with chemiluminescent substrates (Bio-Rad ${ }^{\circledast}$ ) on a Vilber Lourmat gel doc Fusion FX5-XT $\left(\right.$ Vilber $\left.^{\circledast}\right)$. Densitometry analysis was carried out using the FUSION FX software (Vilber $\left.{ }^{\circledR}\right)$. Experiments were repeated at least three times. $t$-test analysis ( $p$-value $\leq 0.05$ ) was used to determine statistically significant results.

\subsubsection{Genetic interaction analysis}

Synthetic genetic analysis was performed in a 384 format as described previously $31,51,165$ to investigate genetic interactions (GIs) of candidate genes. Gl is presumed when double deletion phenotype is different from single deletion ${ }^{53}$, suggesting the deleted genes to be functionally interacting in parallel pathways, causing sick or lethality. Essentially, the candidate gene is deleted in Mat " $\alpha$ " mating type and then crossed to three sets $(\approx 1000)$ of single mutant Mat "a" mating type selected from the single mutant library of yeast. After a few rounds of selection, double gene deletion mutants were selected in Mat a mating type. Fitness or colony size of double mutant strains were compared to that of single mutant strains to detect synthetic sickness or synthetic lethal ${ }^{24,54}$. The experiment was repeated three times. 
For Conditional SGA analysis, we grew our double mutant strain on media with a subinhibitory concentration of $\mathrm{LiCl}(3 \mathrm{mM})$ investigating genes that are expressed only under $\mathrm{LiCl}$ treatment ${ }^{28,33}$.

For Phenotypic Suppression Array (PSA) analysis, we mated Mat " $\alpha$ " carrying overexpression plasmid of our query gene to three sets $(\approx 1000)$ of single mutant Mat "a" selected from the single mutant library of yeast, similar to SGA procedure as mentioned above. In the last step, Mat "a" strains carrying over-expression plasmid are compared to that of Mat "a" carrying a control plasmid ${ }^{34}$, both grown in media treated with $\mathrm{LiCl}$. Possible Gls were selected based on sick or lethal phenotype when grown on media with $\mathrm{LiCl}$ treatment and were compensated with the introduction of over-expression plasmid. In this matter, we can propose a functional connection between these two genes 30,160 .

\subsubsection{Genetic interaction Data Analysis}

Scoring fitness was done using colony size measurement as described previously ${ }^{24,54}$. We set our threshold at fitness $\leq 70 \%$ compared to control. Each experiment was repeated at least three times, and mutual hits observed at least two times were selected. A list of hits then categorized based on their biological process and/or molecular function, with enriched GO terms using Gene Ontology Resources: http://geneontology.org/, Genemania database http://genemania.org and profiling of complex functionality http://webclu.bio.wzw.tum.de/profcom/start.php. 


\subsection{Results}

\subsubsection{Deletion of NAM7, PUS2 and RPL27B increases yeast sensitivity to Lithium Chloride.}

As mentioned above, we know that in PGM2 is an important enzyme in yeast galactose metabolism, which is targeted by $\mathrm{LiCl}$. We also know that over-expression of Translation Initiation Factor TIF2, which codes for elF4A, increases cell resistance to $\mathrm{LiCl}^{148}$. In the spot test assay, we showed that the deletion of TIF2 causes increased sensitivity when grown in the presence of $10 \mathrm{mM}$ LiCl. Deletion of NAM7, PUS2 and RPL27B showed dramatic growth reduction in the same manner (Figure 3.1B). This suggests that the deletion of any of these three genes increases sensitivity to $\mathrm{LiCl}$. It was previously shown that over-expression of TIF2 revert $\mathrm{LiCl}$ toxicity ${ }^{148}$, and to show if our candidate genes would revert $\mathrm{LiCl}$ sensitivity in the same manner, we transferred over-expression plasmid of our candidate genes into deletion strains of their mutants, spotted on $10 \mathrm{mM} \mathrm{LiCl}$. When the plasmids were introduced to the mutants, the fitness of the strains was recovered, proposing they may have a similar function as elF4A in the cell (Figure 3.1C). We showed that indeed, the deletion of TIF2, which codes for elF4A, increased sensitivity to $\mathrm{LiCl}$ in yeast, and it was shown that over-expression of elF4A was able to revert the inhibition of translation due to $\mathrm{LiCl}^{148}$.

These spot test assay findings were confirmed with colony count measurement analysis, providing a quantitative analysis of our results (Figure 3.2). In this experiment, the number of colonies seen in the presence of $\mathrm{LiCl}$ in the media is compared to the number of colonies seen in control media. This is normalized to the wild type. We were able to 
calculate the decreased percentage of colonies using this data. As seen in Figure 3.2, the deletion of NAM7, PUS2, RPL27B, or TIF2 lead to decreased colony formation.

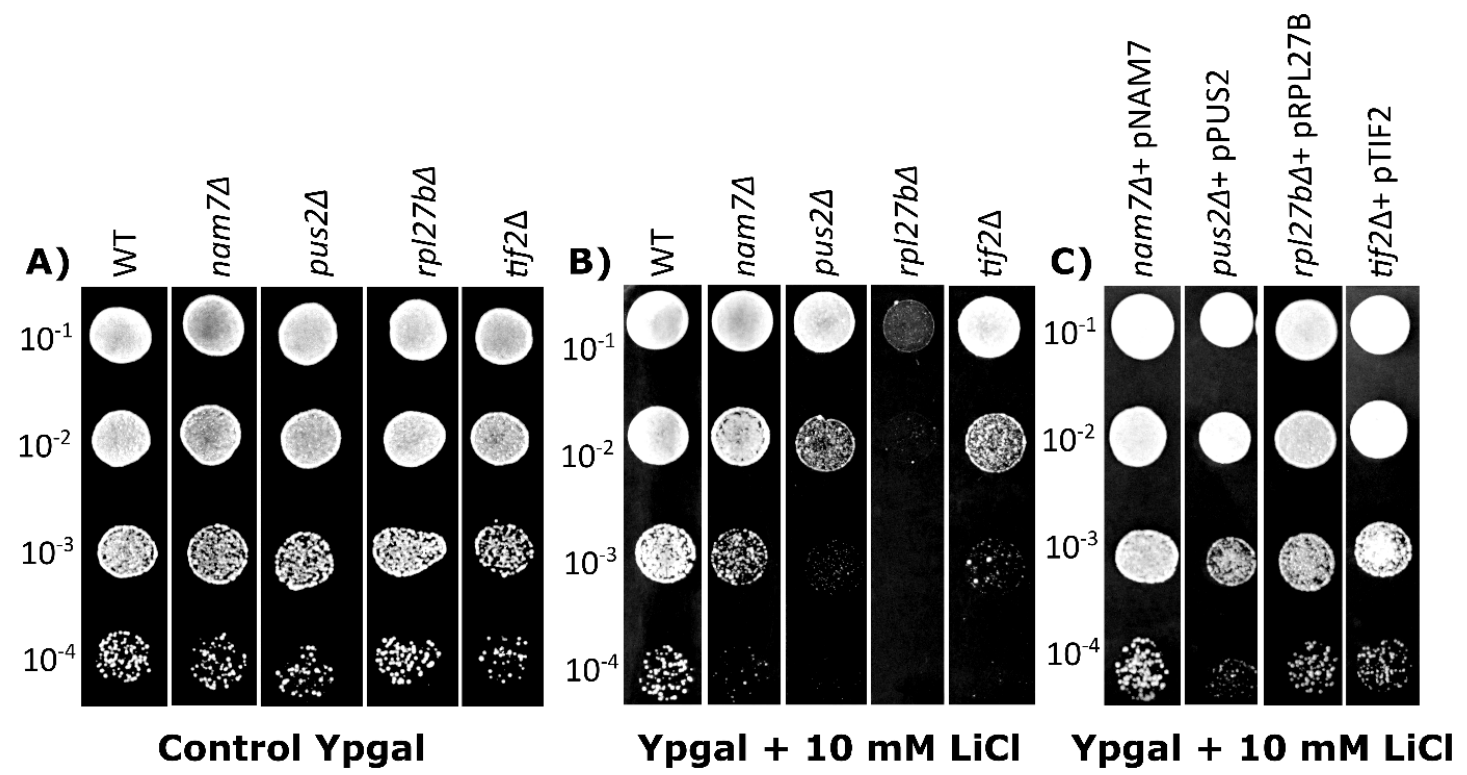

Figure 3.1. Drug sensitivity analysis for different yeast strains using spot test analysis. In (A), (B) and (C) yeast cells were serially diluted as indicated $\left(10^{-1}\right.$ to $\left.10^{-4}\right)$ and spotted on YPgal media with or without $\mathrm{LiCl}(10 \mathrm{mM})$. in (A) and (B) nam7L, pus $2 \Delta$ and $r p / 27 b \Delta$ show less growth under $\mathrm{LiCl}$ treatment. The deletion of TIF2 was used as a positive control. (C) Over-expression of the target gene in their corresponding deletion mutants reverted cell sensitivity to $\mathrm{LiCl}(10 \mathrm{mM})$. 


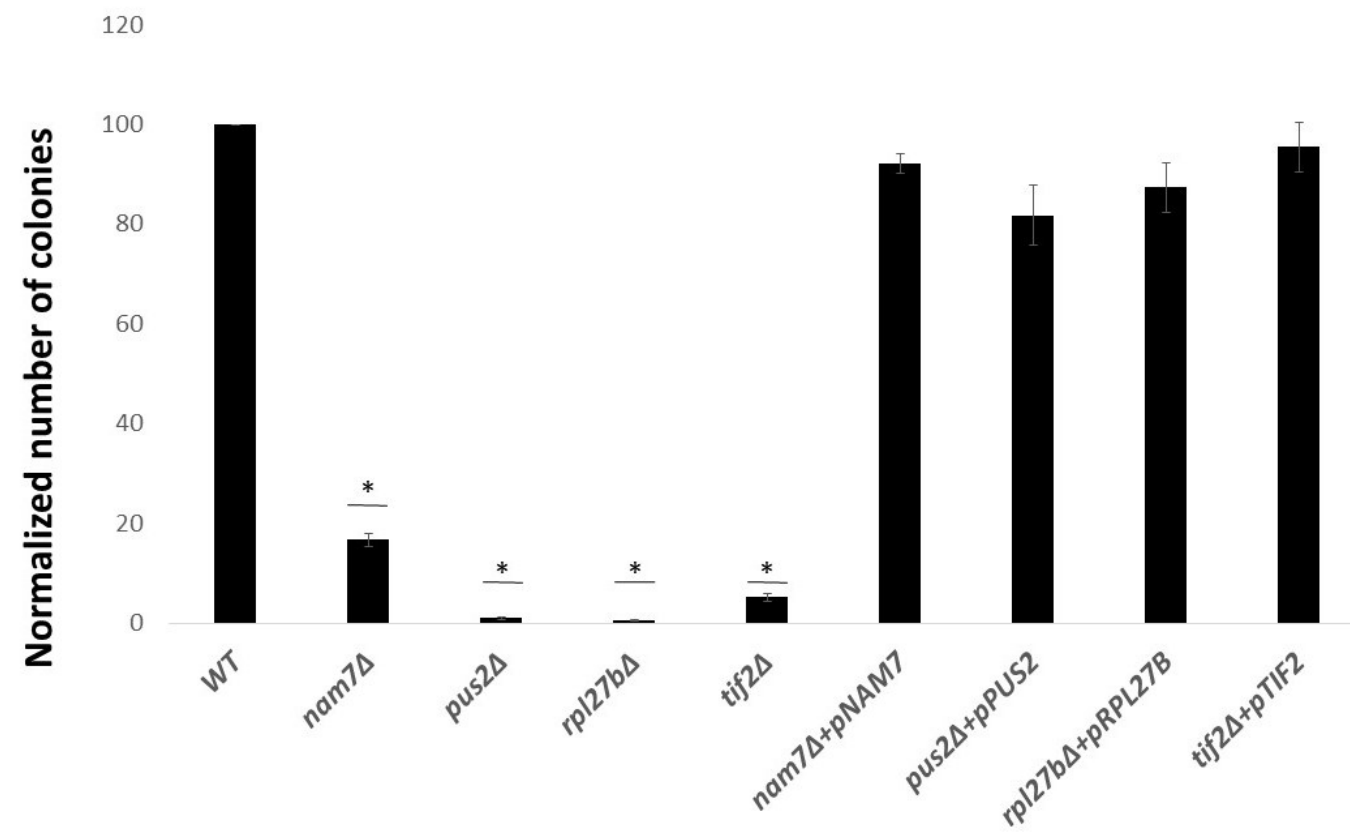

Figure 3.2. Quantitative analysis of drug sensitivity for different yeast strains. The average number of colonies formed for different yeast strains in the presence of $\mathrm{LiCl}$ (10 $\mathrm{mM}$ ) was normalized to that for WT strain. nam $7 \Delta$, pus $2 \Delta$, and rp/27b show fewer colonies under $\mathrm{LiCl}$ treatment. The deletion of TIF2 was used as a positive control. Overexpression of target genes in their corresponding deletion mutants reverted cell sensitivity to $\mathrm{LiCl} .{ }^{*}(P$-value $\leq 0.05)$ represent statistically significant results from WT control. Data represents the average from three independent experiments and error bars represent standard deviation.

\subsubsection{NAM7, PUS2, and RPL27B regulate the expression of PGM2 at the level of translation.}

Previous studies have shown that $\mathrm{LiCl}$ exposure alters the expression of $P G M 2$, a phosphoglucomutase crucial to galactose metabolism ${ }^{148-150}$. This enzyme plays two important roles: the facilitation of galactose into glycolysis and the conversion of glucose1-phosphate to glucose-6-phosphate.

Since altered expression of PGM2 has been seen in the presence of $\mathrm{LiCl}$, we examined to see if NAM7, PUS2, or RPL27B would affect PGM2 expression at the translation level. 
Pgm2p was tagged with GFP and western blot analysis was done to see if any of the three genes altered PGM2 expression (Figure 3.3A). When cells were exposed to $10 \mathrm{mM} \mathrm{LiCl}$, the deletion of any three of the genes resulted in reduced protein levels of Pgm $2 \mathrm{p}$. When cells were not exposed to $\mathrm{LiCl}$, the deletion of NAM7, PUS2, or RPL27B showed no observable difference.

To see if NAM7, PUS2, or RPL27B impact PGM2 transcription levels, qRT-PCR was performed to measure the expression of PGM2 mRNA. As seen in Figure 3.3B, the deletion of NAM7, PUS2, or RPL27B did not significantly alter PGM2 mRNA levels when exposed to $\mathrm{LiCl}$, suggesting that these genes do not impact PGM2 at the transcriptional level. Thus, this would imply that NAM7, PUS2, or RPL27B affect the expression of Pgm2p at the protein level. These results are in agreement with a previous study by Sofola-Adesakin ${ }^{145}$ which demonstrated that $\mathrm{LiCl}$ impaired gene expression during protein synthesis and not during transcription. 

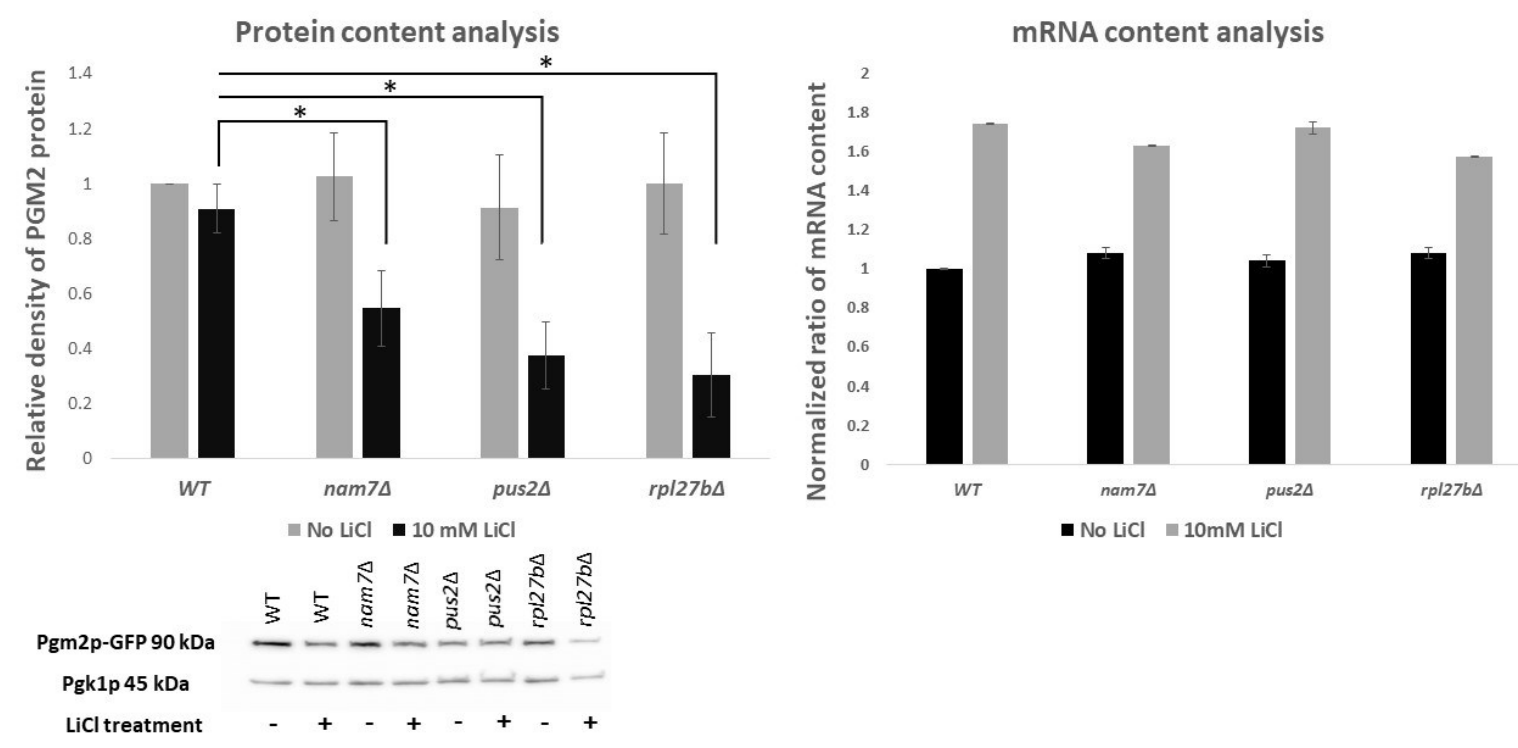

Figure 3.3. Protein and mRNA content analysis. (A) Protein content analysis of Pgm2pGFP protein in deletion of yeast strains for nam7 7 , pus $2 \Delta$, and $r p / 27 b \Delta$. Western blot analysis was used to measure the protein content for Pgm2p-GFP protein in the absence or presence of $\mathrm{LiCl}(10 \mathrm{mM})$. Pgk1p was used as a housekeeping gene and the values are normalized to that. (B) mRNA content analysis of PGM2 in nam7 4 , pus $2 \Delta$, and $r p / 27 b \Delta$. qRT-PCR was used to evaluate the content of $P G M 2$ mRNA in yeast gene deletion mutants related to WT strain and normalized to PGK1 mRNA levels. mRNA level shows to increase in treatment of $\mathrm{liCl}$ but there is no significant difference between mutants and WT. Each experiment was repeated at least three times. * $(P$-value $\leq 0.05)$ represent statistically significant results compared to WT coontrol. Error bars represent standard deviation.

\subsubsection{Translation of 6-galactosidase reporter mRNA with a hairpin structure is altered by NAM7, PUS2, and RPL27B.}

Previous studies have predicted that the $5^{\prime}$ end of PGM2 mRNA has a highly structured region ${ }^{163}$ (Appendix Figure 8.1). PGM2 expression is severely reduced in the absence of translation initiation helicase TIF2, a protein responsible for unwinding mRNA structures during translation ${ }^{148}$. Since we saw that NAM7, PUS2, and RPL27B are likely to work at 
the translational level, we wanted to see if these genes are possibly impacting the translation of highly structured mRNAs. For this experiment, we inserted 5'-UTR of PGM2 in form of LacZ cassette of p416 plasmid (pPGM2). Then pPGM2 was transformed into deletion of mutant strains of our candidate genes and WT and 6-galactosidase activity was measured as a reference for translation activity (Fig 4.4A). It was shown that translation activity significantly decreased in Nam7 4 , Pus $2 \Delta$, and $R p / 27 b \Delta$ carrying a plasmid with hairpin when compared to translation rate with the control plasmid (Figure 3.4B).

To support our findings, we used another construct with a strong hairpin structure at $5^{\prime}$ UTR (p281-4) ${ }^{157}$ to show if query genes were most likely affecting highly structured $5^{\prime}$ UTRs and not only PGM2. Our control was p281 without the hairpin structure ${ }^{157}$. We observed that with the deletion of NAM7, PUS2, or RPL27B, 6-galactosidase activity was reduced in p281-4, whereas p281 had no observable difference (Figure 3.4A and 3.4B). These results demonstrate that these genes are not affecting the general translation of mRNAs lacking highly structured mRNAs. 

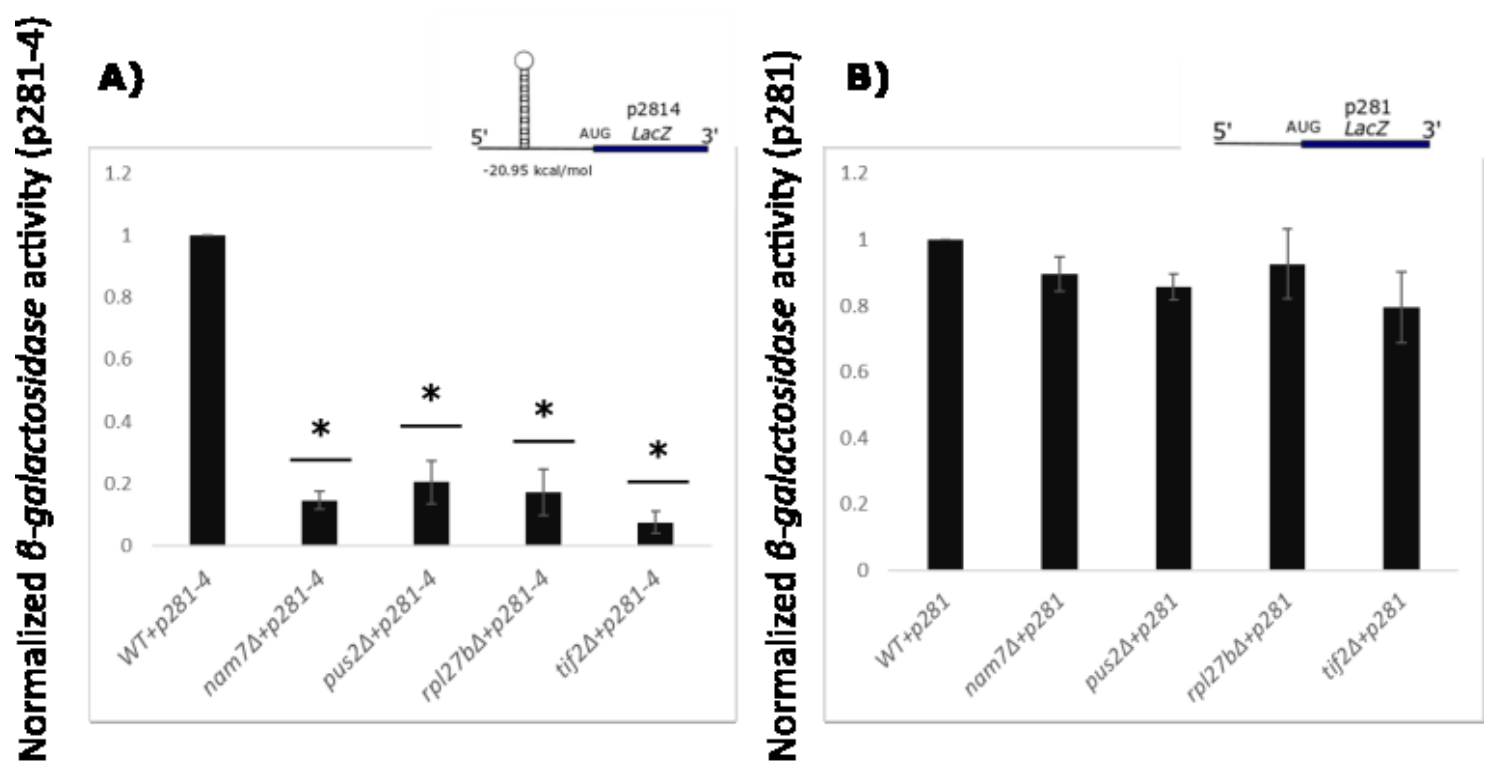

Figure 3.4. 6-galactosidase expression analysis in different yeast strains. (A) Activities from 6-galactosidase mRNAs that carry a strong hairpin structure (p281-4) upstream of a $L a C Z$ reporter were highly reduced in nam $7 \Delta$, pus $2 \Delta$, and hal $1 \Delta$ strains; tif2 $\Delta$ was used as a positive control. (B) Strains carrying low complexity (p281) upstream of the LacZ gene did not show reductions in 6 -galactosidase activity. Values are normalized to that for WT. Each experiment was repeated at least 3 times and error bars represent standard deviation. ${ }^{*}(P$-value $\leq 0.05)$ represent statistically significant results from WT control. Insets represent schematic representation for the structure of the reporter mRNAs.

Since NAM7, PUS2, and RPL27B impacted translation of structured reporter mRNAs, we investigated if they were able to impact the translation of other naturally structured mRNAs. To do this, we designed two 6-galactosidase mRNA reporters with different complex RNA structures. PTAR has the 5'-UTR of the HIV1-tar gene, which has a strong hairpin loop, while pRTN has the 5'-UTR of the FOAP-11 gene, which has a highly structured region ${ }^{102,164}$. When NAM7, PUS2, or RPL27B were deleted, levels of $B$ galactosidase expression were significantly reduced (Figure 3.5). 

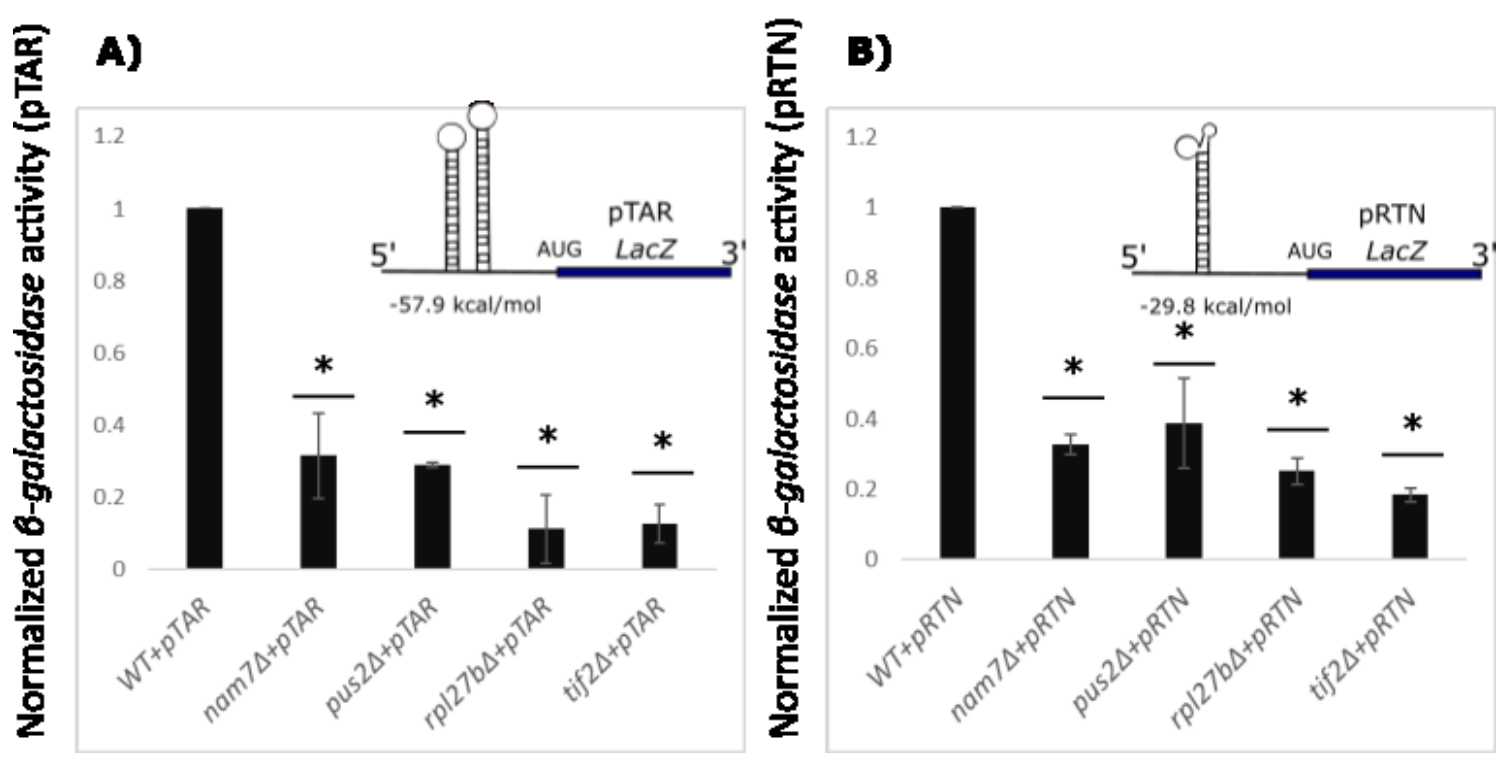

Figure 3.5. Normalized 6-galactosidase activity is lower in nam7 for structured mRNAs. pTAR (A) and pRTN (B) constructs contain the highly structured 5'UTR of HIV1-tar and FOAP-11 genes, respectively, in front of the 6 -galactosidase reporter mRNA. Values are normalized to that for $W T .{ }^{*}(P$-value $\leq 0.05)$ represent statistically significant results from WT control. Each experiment was repeated at least 3 times and error bars represent standard deviation.

\subsubsection{Genetic interaction analysis further connects the activity of NAM7, PUS2, and} RPL27B to protein biosynthesis.

Genetic interaction (GI) analysis assumes that parallel pathways allow for plasticity and tolerance against random deleterious mutations, protecting cells from certain death if one gene is deleted or mutated in a pathway ${ }^{24}$. This means that a gene in one pathway can compensate for the lack of gene activity in a parallel pathway, allowing the cell to survive. Accordingly, when two genes in parallel pathways are deleted, cell fitness decreases or the cell dies. As a result, we can propose they are having genetic interaction, or in other words, they are functionally working in parallel pathways called negative genetic interactions (nGIs). nGls are useful in many studies to understand gene function 
$29,31,34$

Analysis of Gls in yeast is done by mating two types of yeast: $\alpha$-mating type (Mat $\alpha$ ), and a-mating type (Mat a). Mat $\alpha$ is the target gene deletion, which is crossed with Mat a, an array of single-gene deletions to produce double gene deletions ${ }^{51}$. The fitness of double deletions is measured using colony size assessment ${ }^{165}$. Using this method, we made double deletions for our three genes, observing the genetic interactions of NAM7, PUS2, and RPL27B with nearly 1000 other genes that may interact with our candidates. In this experiment, 384 genes were randomly selected form the mutant library as controls.

We observed 33 nGls with NAM7, including PRP22, TIF2, GCD11, and PRT1. PRP22 is a DEAH-box RNA helicase, TIF2 codes for the translation initiation factor elF4A, GCD11 forms part of the small subunit of elf2, and PRT1 is the subunit of elf3. Overall, $36 \%$ of the genes that had nGIs with NAM7 are involved in translation, many of which are involved in translation initiation factor activity including YGR054W and HCR1 (Figure 3.6).

PUS2 had 35 nGls including DHH1, EAP1, and HCR1. DHH1 codes for an ATP-dependent RNA helicase. EAP1 codes for an elF4E-associated protein, and HCR1 codes for a subunit of the elF3 translation initiation factor, which is also important in the binding of initiation factors to the 40 S subunit and AUG recognition along with HCR1 as an RNA recognition motif ${ }^{180} .31 \%$ of PUS2 nGls were involved in ribosomal structural units. XRN1, YOR302W, VIP1, and WHI3, along with the other mentioned genes, are involved in the regulation of translation (Figure 3.6). 
$R P L 27 B$ had 54 nGls, $25 \%$ of which are involved in translation and $16 \%$ of which are involved in translation regulation (Figure 3.6). This included $D H H 1, E A P 1, S L H 1$ and SKI2 with RNA helicase activity, and ECM32 as a DNA helicase enzyme, modulating translation termination.

Comparing NAM7, PUS2, and $R P L 27 B$ using a genetic interactor revealed some interesting mutual hits being important mainly in translation regulation. HCR1 is a subunit of elF3 and is known to be important for scanning efficiency especially in cooperation with DED1 (RNA helicase) on long $5^{\prime}$-UTRs ${ }^{131}$ and binding with DHX29 in human cells ${ }^{181}$. HCR1, another mutual hit, and PRT1 are crucial in recognition of the right start codon during scanning by inhibiting leaky scanning through promoting the stability of ribosomes on mRNA ${ }^{182,183}$. XRN1 is known to be involved in mRNA decay and transcription regulation but recently was proposed that it might also play a role in the translation pathway by regulating translation of specific mRNAs through binding to elF4F complex a translation IC ${ }^{184}$. DED1 is an ATP-dependent helicase that associates with elF4A to regulate translation initiation ${ }^{94,134}$. Methylation of DED1 strengthens its binding to elF4A and to XRN1 ${ }^{101}$, suggesting their potential effect on translation regulation. XRN1 was also proposed to interact with another helicase $\mathrm{DHH} 1$ in yeast to control the negative regulation of translation by decapping mRNAs for degradation 97,185 . P54 (homolog of DED1 in humans) was shown to be important in localization and assembly of $\mathrm{P}$-bodies in the cell ${ }^{97}$. 


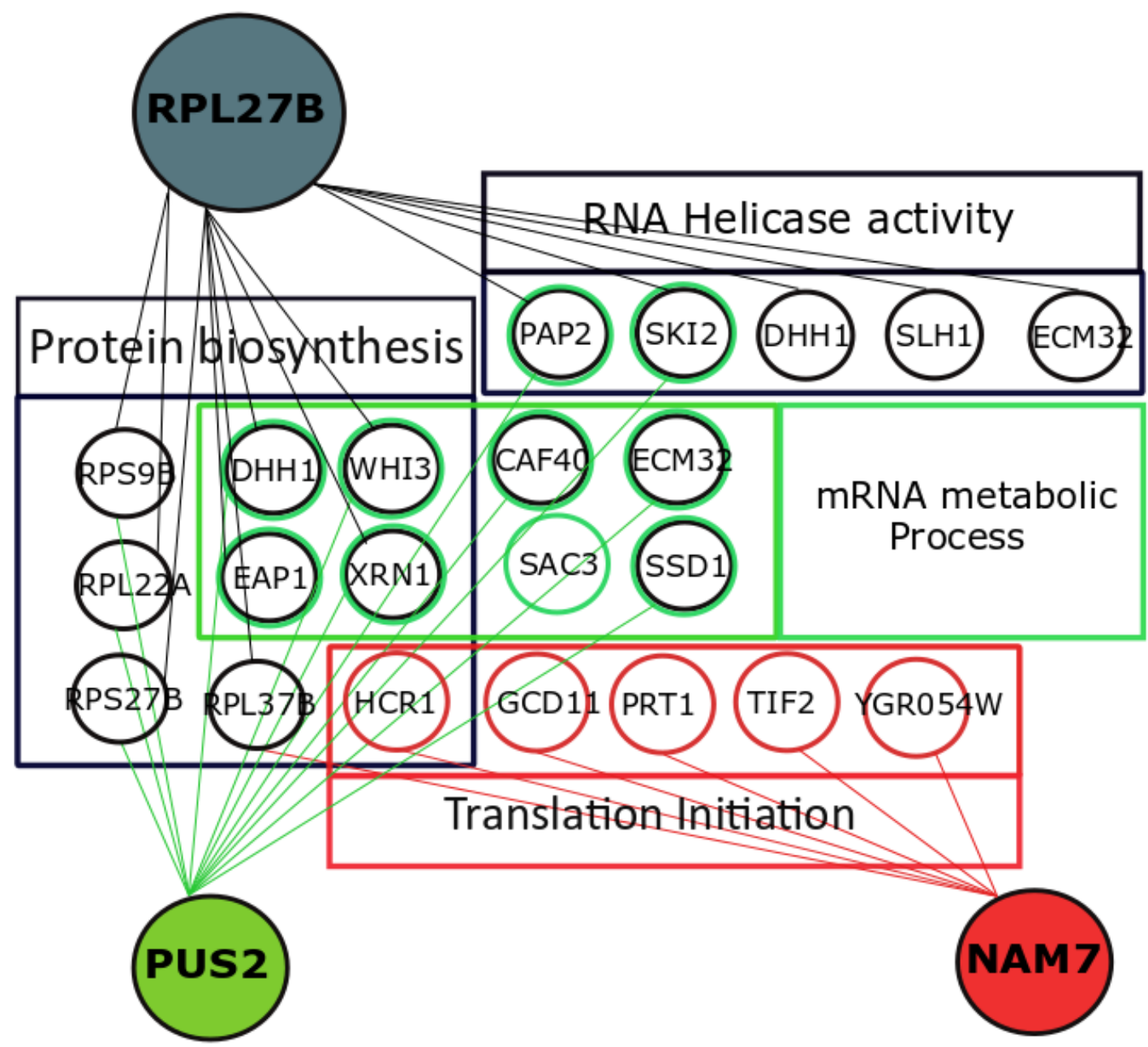

Figure 3.6. nGls for NAM7, PUS2, and RPL27B. Our data shows a cluster of interactors involved in protein biosynthesis pathway for NAM7 $(\mathrm{P}=1.6 \mathrm{e}-07)$ PUS2 $(\mathrm{P}=4 \mathrm{e}-08)$ and $R P L 27 B(\mathrm{P}=1.2 \mathrm{e}-05)$. RPS9B, RPL37B, RPS7B, RPL22A, DHH1, EAP1,WHI3, and XRN1 are mutual hits shared between NAM7, PUS2, and RPL27B. Circles represent genes, lines represent $\mathrm{nGls}$ identified in this study, black circles showing $\mathrm{nGls}$ for $R P L 27 B$, green circles showing PUS2 and red are NAM7.

Another form of nGls can be studied under the sub-inhibitory concentration of drugs to investigate possible candidate genes being activated under specific conditions called conditional SGA ${ }^{28,33}$. We were looking for the regulation of candidate genes important in translation control under LiCl toxicity. For this experiment, we studied SGA analysis for 
NAM7, PUS2, and RPL27B under the sub-inhibitory concentration of $\mathrm{LiCl}$ (3 $\mathrm{mM}$ ). Shown in Figure 3.7, new nGls were observed for our candidate genes which are involved in the translation pathway but mostly translation regulation, indicating their roles in response to $\mathrm{LiCl}$ toxicity in the cell. $B C K 1, C T K 1$, and $M C K 1$ were the mutual interactors between our genes which have been shown to be important in translational control, specifically for $\mathrm{LiCl}$ toxicity in our case. $B C K 1, E A P 1$, and POP2 (also nGIs) are important in the negative regulation of translation under stress conditions. They are important in deadenylation and decapping of mRNAs to be degraded in correlation with $D H H 1$, an important RNA helicase in the MAP kinase pathway to regulate the expression of specific genes ${ }^{169,171-173}$. On the other hand, CTK1 is a conserved kinase that phosphorylate RPS2p, one of the components of small ribosomal subunit, affecting translation fidelity during elongation as well as phosphorylation of other translation initiation factors including eIF4A, eIF5, elF4G, and elF3, to improve their function as crucial factors of proper translation initiation ${ }^{186,187}$. It was shown that elF4A physically interacts with cyclin-dependent kinase A (homolog of CTK1 in plants) which is important in cell proliferation and cell cycle ${ }^{80}$ is also a target of $\mathrm{LiCl}$. Another interesting mutual hit we observed in these screens was MCK1, which happens to be involved in GSK-3 pathway, one of the main targets of $\mathrm{LiCl}$ aside from the Protein Kinase C pathway ${ }^{146}$. 


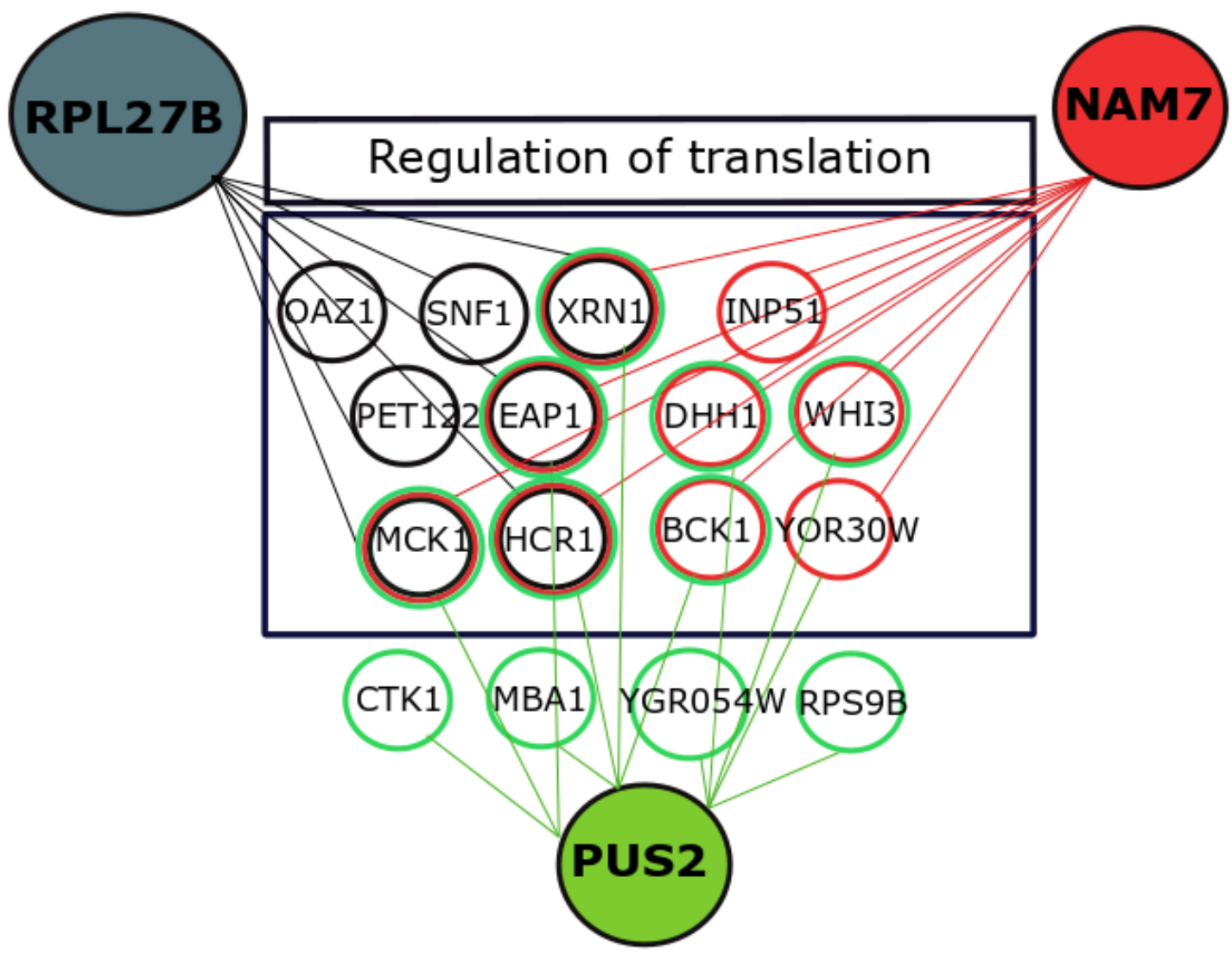

Figure 3.7. Conditional nGls for NAM7, PUS2, and RPL27B under $3 \mathrm{mM}$ concentration of $\mathrm{LiCl}$. Our data shows a mutual GO term cluster of translation regulation genes, with $B C K 1, M C K 1, C T K 1, E A P 1$ and $D H H 1$ as mutual nGls between NAM7 (P=0.00037), PUS2 $(\mathrm{P}=1.1 \mathrm{e}-06)$ and $R P L 27 B(\mathrm{P}=1 \mathrm{e}-06)$, apart from genes involved in translation (not shown). Circles represent genes and lines represent nGls identified in this study, black circles showing nGIs for RPL27B, green circles showing PUS2 and red are NAM7.

PSA analysis focuses on another form of interaction where over-expression of one gene compensates for the absence of another gene under drug treatment $7,31,34,168$. In our case, we treated our arrays of mutant strains with $10 \mathrm{mM} \mathrm{LiCl}$ and consequently, some showed sensitivity. Then we were able to revert $\mathrm{LiCl}$ sensitivity of a number of deletions by introducing overexpression plasmid of $N A M 7, P U S 2$, and $R P L 27 B$. We were able to find some interesting mutual hits, in which over-expression of either NAM7, PUS2 or RPL27B 
compensated for their deletion in treatment with $10 \mathrm{mM} \mathrm{LiCl}$. These including YGR0564W (elF2), DPH2, EAP1, TMA7 and ITT1, which all involved in the translation pathway and posttranscriptional regulation of gene expression. All of these genes have been shown to interact with $D H H 1$, a known RNA helicase with different roles in unwinding secondary structure of mRNA, mRNA decapping and translational repression ${ }^{173}$. Eap1, an elF4Eassociated protein that accelerates decapping of mRNA, is an example of a protein that correlates with $D H H 1$ in negative regulation of translation ${ }^{171}$. 


\section{A correlation between $3^{\prime}$-UTR of OXA1 gene and yeast mitochondrial translation.}

\subsection{Abstract}

Mitochondria possess their own DNA (mtDNA) and they are capable of carrying out transcription and translation of mt-encoded ORFs. Although protein synthesis takes place in mitochondria, the majority of the proteins in mitochondria are of nuclear origin. Untranslated $3^{\prime}$ and $5^{\prime}$ regions of mRNAs ( $3^{\prime}-U T R$ and $\left.5^{\prime}-U T R\right)$ are thought to play key roles in directing and regulating the activity of mitochondria mRNAs. Here we investigate the association between the presence of $3^{\prime}$-UTR from the OXA1 gene on a prokaryotic reporter mRNA and mitochondrial translation in yeast. OXA1 is a nuclear gene that codes for mitochondrial inner membrane insertion protein and its $3^{\prime}$-UTR is shown to direct its mRNA towards mitochondria. Using a 6-galactosidase reporter gene we provide genetic evidence for a correlation between the presence of $3^{\prime}$-UTR of OXA1 on mRNAs and mitochondrial translation in yeast.

\subsection{Introduction}

Diverse functions of mitochondria can vary from cell to cell. In addition to its function in signaling pathways and metabolism, the most predominant role of mitochondria is to produce energy for cell survival in the form of adenosine triphosphate (ATP). Mitochondrial activities are facilitated by mitochondrial proteins encoded by either nuclear DNA or maternally-inherited mtDNA ${ }^{188}$. Like nuclear DNA, mtDNA goes through DNA replication, transcription and translation ${ }^{189}$. The machineries involved in these 
processes, however resemble those of prokaryotic organisms reflecting the evolutionary origin of mitochondria ${ }^{190}$. DNA damage, heat shock, UV radiation and other stimuli including chemotherapeutic drugs can affect mitochondria-driven apoptosis ${ }^{191}$.

In a number of neurological disorders including Parkinson's and Alzheimer's diseases, mutations in the respiratory chain and OXPHOS system, coded by mt-DNA can lead to premature cell death ${ }^{192}$. Also, patients with certain mitochondrial disorders are shown to have impaired immune systems and a higher probability of infections ${ }^{193}$. Other mitochondrial associated disorders include diabetes and a number of cancers ${ }^{194 .}$ Targeting mitochondrial biosynthesis and limiting mitochondrial reactive oxygen species (ROS) production is thought to be a promising cancer therapeutic strategy ${ }^{193}$. In this context, targeting different agents including proteins, DNA, mRNA, nano-agents, and antioxidants to mitochondria has been proposed for therapeutic purposes ${ }^{195,196}$.

Like in humans, in Saccharomyces cerevisiae, mtDNA carries a limited number of genes involved in gene expression, and respiratory chain, in addition to several ribosomal proteins, tRNAs, $12 \mathrm{~S}$ and $16 \mathrm{~S}$ rRNA. ${ }^{197}$. The majority of the proteins that function within this organelle are of nuclear origin and are imported to mitochondria ${ }^{188}$. Certain nuclear RNAs including $5 \mathrm{~S}$ ribosomal RNA and microRNAs are also reported to enter mitochondria 198. In a yeast genome-wide analysis 466 nuclear genes were identified that when deleted caused impaired mitochondria respiration. Human homologs of many of these genes were linked to human mitochondria disorders, indicating that yeast may serve as a suitable model organism to study mitochondria-associated human diseases ${ }^{199}$. 
It is generally accepted that the localization of certain nuclear mRNAs to the vicinity of mitochondria leads to the localized translation of these nuclear genes near mitochondria. This is followed by the import of the newly translated proteins into mitochondria ${ }^{188}$. An example of these mRNAs is that of the OXA1 gene, which codes for mitochondria inner membrane insertion protein. The $3^{\prime}$-UTR of the OXA1 gene was shown to direct the mRNA to the vicinity of mitochondria ${ }^{200}$. It is suggested that this $3^{\prime}$-UTR sequence serves as a binding site for certain RNA-binding proteins that, with the help of motor proteins, transport mRNAs towards mitochondria ${ }^{188}$. Then, OXA1 mRNA is translated near the outer surface of the mitochondria. This is followed by the import of the newly synthesized Oxa1p into mitochondria ${ }^{201}$. In this way, the presence of Oxa1p in mitochondria is directly influenced by the 3'-UTR of its mRNA. However, from the current data, it is not clear if a portion of OXA1 mRNA molecules can also enter mitochondria. The main reason for this lack of knowledge stems from technical limitations associated with purification experiments. An inherent limitation of purification procedures is that they all suffer from different degrees of co-purifying contaminants. Consequently, deriving precise conclusions from purification experiments alone is not an easy task. There is often a need for designing unique approaches to answer specific questions. OXA1 mRNA is known to be abundant and efficiently translated in the cytoplasm of yeast, with the currently available methodologies, it is very challenging to differentiate whether a small portion of OXA1 mRNA may also get into and translated in mitochondria. 
In the current study, we investigate the $3^{\prime}$-UTR of OXA1 mRNA for its possible translation in the mitochondria. We designed an approach that uses the foundations that govern mitochondrial translation as the basis to evaluate the translation of a prokaryotic reporter B-galactosidase mRNA inside mitochondria. An important feature of our approach is that the construct is designed to hinder the cytoplasmic translation of its mRNA. Our observations provide genetic evidence for a correlation between 3'-UTR of OXA1 mRNA and the translation of 8 -galactosidase mRNA by mitochondrial translation machinery.

\subsection{Materials and Methods}

\subsubsection{Strains plasmids and media}

The baker's yeast, S. cerevisiae strain BY4741 was used in this study. The expression vector p416GALL 32 was utilized for expression analysis. This vector contains a galactokinase promoter (GALL-pro) upstream of a lacZ reporter gene that was cloned as an Xbal/BamHI cassette. The plasmid also contains URA3 and Ampicillin resistance (Amp)

genes for selective growth of yeast and E. coli, respectively in selective media ${ }^{33}$. $\mathrm{DH} 5 \alpha$ strain of E. coli was used to propagate plasmids. Standard rich (YPD) and synthetic complete (SC) media were used for growth of yeast except if specified otherwise. LB (Lysogeny broth) was used to grow E. coli. Antibiotics were used in the following concentrations: ampicillin, $100 \mu \mathrm{g} / \mathrm{ml}$; cycloheximide, $45 \mathrm{ng} / \mathrm{ml}$; and chloramphenicol, $1.4 \mathrm{mg} / \mathrm{ml}$. 


\subsubsection{DNA manipulations}

3'-UTR of OXA1 was amplified by PCR from genomic template using the following primers: 3'-YOXAIUTR-F (5' CGCGGATCCATTAATAACAAAAAATGAATAAAGGC 3') and 3'-YOXAIUTRR (5' CGCGGATCCTCCAAATGATTATTTCAAGCAATAAA 3'). The resulting PCR product was digested by BamHI and ligated into the unique BamHI site of p416GALL. Sequencing was used for confirmation. Designed 5'-UTRs of COX2 were cloned by inserting synthesized double stranded DNA into the unique Xbal site of p416GALL plasmid. The following pairs of primers were synthesized and hybridized in ligation buffer. Primers 5COXa (5' AATAGTATTAACATATTATAAATAGACAAAAGAGTCTAAAGGTTAAGATTTATTAAAATGC $\quad 3{ }^{\prime}$ ) and 5COXb (5' CTAGGCATTTTAATAAATCTTAACCTTTAGACTCTTTTGTCTATTTATAATATGTT AATACTATTACGCCATGGTCAGCTTACGCCCGCCTGTTTGGCGGGCGTAAGCTGG $\quad 3^{\prime}$ ) were used for MT-C1 construct and primers 5COXDa (5' CTAGCCAGCTTACGCCCGCCAAA CAGGCGGGCGTAAGCTGACCATGGCGTAATAGTATTAACATATAGATCTTAGACAAAAGAGTCT AAAGGTTAAGATTTATTAAAATGC 3') and 5COXDb (5' CTAGGCATTTTAATAAATCTTAACCTT TAGACTCTTTTGTCTAAGATCTATATGTTAATACTATTACGCCATGGTCAGCTTACGCCCGCCTGT TTGGCGGGCGTAAGCTGG 3') were used for MT-C1X construct. Sequencing was used for confirmation. Plasmids were extracted from transformed cells via QIAprep Miniprep kit, using the manufacturer's specifications.

\subsection{3 qRT-PCR}

cDNA synthesis of mRNA samples was processed according to Bio-Rad RT-PCR kit. 
Quantitative PCR was performed using iQSYBR Green master-mix kit (Biorad) according to the manufacturer's instructions. qPCR amplification and detection were performed on RT-PCR cycler (ROTOR GENE RG-3000 from Corbett research). Data were analyzed using Rotor-Gene Real-Time Analysis Software 6.0.14.

\subsubsection{6-galactosidase liquid assay}

The quantitative 6 -galactosidase assay was performed using ONPG (O-nitrophenyl-a-Dgalactopyranoside) method as described ${ }^{30}$. Cells were induced by exposure to $2 \%$ galactose for $6 \mathrm{hrs}$. As needed, cycloheximide or chloramphenicol was added to the induction media. When required yeast mitochondria were isolated using BD Free Flow Electrophoresis (FFE) system according to the manufacturer's specifications.

\subsection{Results and Discussion}

\subsubsection{In isolated mitochondria, the activity and content of a 6 -galactosidase mRNA is correlated to $3^{\prime}$-UTR of OXA1 gene}

mRNA targeting is generally regarded as a component of localized translation. The $3^{\prime}$-UTR of OXA1 mRNA is believed to mediate the targeting of this mRNA to the vicinity of mitochondria where the mRNA is translated and subsequently imported into the mitochondria ${ }^{202}$. OXA1 codes for a mitochondria inner membrane protein that is thought to play a role in membrane binding of ribosome and insertion of newly synthesized polypeptides into mitochondrial inner membrane ${ }^{190}$. Also, there are examples of numerous RNA molecules that are imported into mitochondria in different organisms. Various mitochondrial tRNAs are encoded by the nuclear genome and subsequently 
targeted into mitochondria ${ }^{130}$. More recently, a 20 nucleotide sequence from the $H 1$ RNA, the RNA component of RNase P enzyme, was shown to mediate the import of fusion tRNA and mRNA molecules into mitochondria and rescuing phenotypic defects in two different human cell lines caused by defective tRNAs ${ }^{195}$. Since OXA1 3'-UTR is shown to target mRNAs to the vicinity of yeast mitochondria, we asked the question if this $3^{\prime}$-UTR might also direct the mRNA into mitochondria. For this purpose, we designed a specific approach that utilized the foundations that govern mitochondrial translation and architected a distinct prokaryotic reporter B-galactosidase construct. This construct, termed MT-C1 for Mitochondrial Translation Construct 1, is designed to extensively hinder cytoplasmic translation of its reported mRNA and enhance its translation within mitochondria (Figure 4.1). At its $3^{\prime}$-end, MT-C1 carries OXA1 $3^{\prime}$-UTR. It's 5'-UTR is designed to impede cytoplasm translation by including the following elements. 1 . A strong inhibitory secondary structure with an estimated $\Delta \mathrm{G}$ value of $-28 \mathrm{kcal} \mathrm{mol}^{-1}$ previously shown to prevent the advancement of $40 \mathrm{~S}$ ribosomes along mRNA and thereby inhibiting translation ${ }^{203}$. The purpose of this structure is to prevent the scanning of the cytoplasm ribosomes from 5'-CAP towards the start codon. 2. An early initiation start codon next to a consensus Kozak sequence. The presence of a strong Kozak sequence will promote an out-of-frame cytoplasmic translation initiation for those ribosomes that may escape the inhibitory structure. These ribosomes will start translation from the out of frame AUG codon. And 3. consecutive premature stop codons downstream of the early start codon. These stop codons can terminate unwanted translations mediated by its upstream region. The mitochondrial translation is reported to require a consensus translation initiation 
sequence 5'-UAUAAAUA-3'. To promote mitochondrial translation, 5'-UTR from COX2 mRNA, which contains the mitochondria consensus translation initiator sequence, was incorporated upstream of the AUG start codon of a prokaryotic B-galactosidase reporter gene. COX2 codes for cytochrome $\mathrm{C}$ oxidase subunit II, the terminal member of the $\mathrm{mt}$ inner membrane electron transport chain and is translated in mitochondria ${ }^{204}$. It is one of the major mitochondrial mRNAs and has a well-characterized mitochondrial 5'-UTRs and the shortest (54 nucleotides) reported ${ }^{204,205}$. A second construct termed MT-C2 was designed as a control. It contains all the features of MT-C1 with the exception that it lacks the $3^{\prime}$ - UTR of OXA1 at its 3'-end.

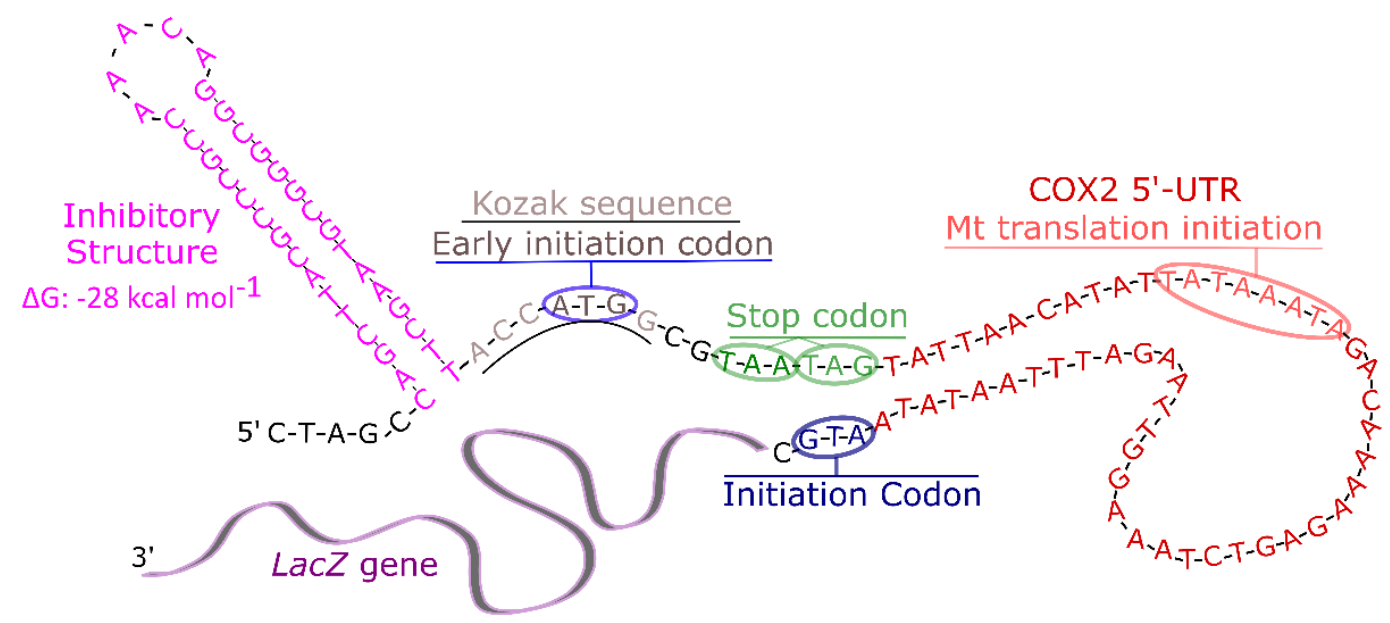

Figure 4.1. Schematic representation of the B-galactosidase mRNA designed to limit cytoplasmic translation. Various features of the 5'-UTR for this mRNA are shown in different colors. Elements designed to limit cytoplasmic translation include an inhibitory structure, an early initiation codon within Kozak sequence and early tandem stop codons. COX2 5-UTR mediates mitochondrial translation. Figure created using Inkscape.

MT-C1 and MT-C2 were used to transform yeast cells. Mitochondria were isolated from the transformed cells and the 6 -galactosidase mRNA content, as well as 6 -galactosidase 
activities of the isolated samples, were measured (Figure 4.2). Represented in Figure 4.2a, 6-galactosidase mRNA analysis using RT-qPCR indicated that mitochondrial mRNA content for those mRNAs lacking OXA1 3'-UTRs (MT-C2) is approximately reduced by $90 \%$ in comparison to those carrying OXA1 3'-UTR (MT-C1). Similarly, 6-galactosidase activity measurement showed an approximately $85 \%$ reduction for mRNAs lacking OXA1 3'-UTR.
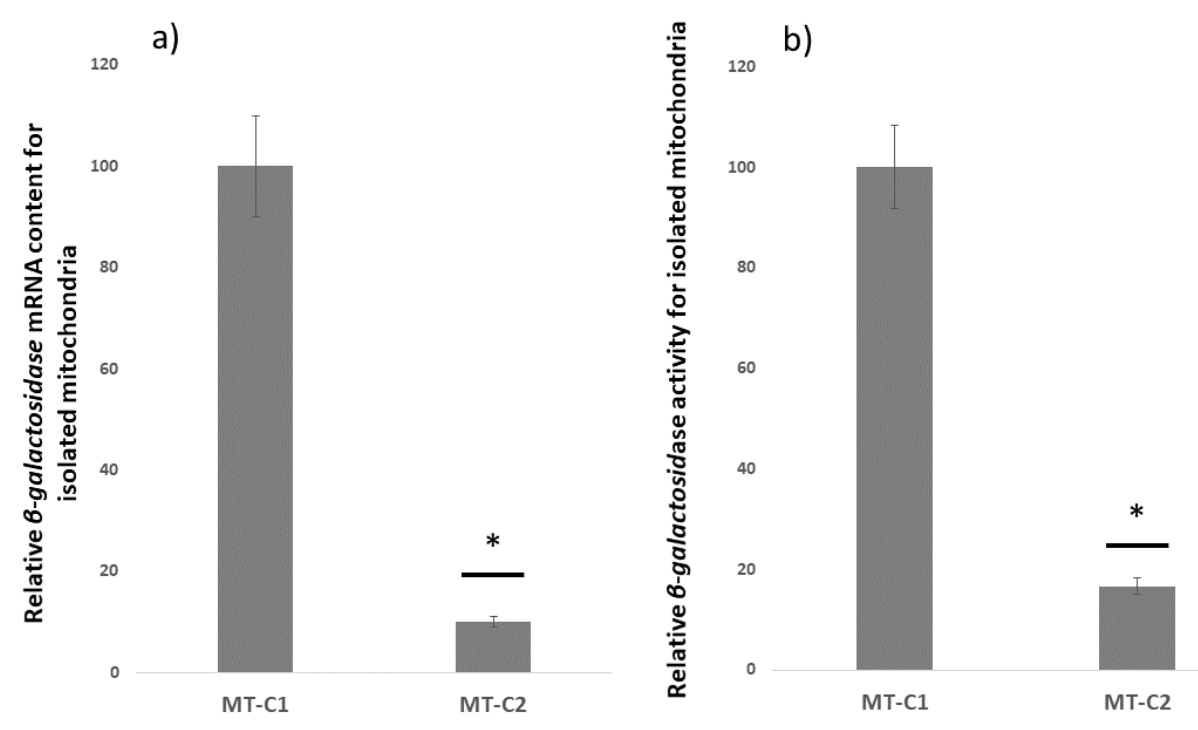

Figure 4.2. mRNA content and 6-galactosidase expression analysis of isolated mitochondria from different yeast strains. a) RT-PCR analysis indicated that in the absence of OXA1 3'-UTR (MT-C2) B-galactosidase mRNA content is significantly reduced in isolated mitochondrial. The values are normalized to that of the MT-C1 construct that carries OXA1 3'-UTR. PGK1 mRNA was used as a control and all values are related to that. Each experiment is repeated at least 3 times. Error bars represent standard deviation. b) 6-galactosidase activity of isolated mitochondria is highly reduced in the absence of OXA1 $3^{\prime}$-UTR (MT-C2). Values are normalized to that for MT-C1 set at $100 .{ }^{*}$ represent statistically significant results ( $P$-value $\leq 0.05)$ compared to the WT. Each experiment is repeated at least three times. Error bars represent standard deviation.

In these experiments, MT-C2 may represent the background levels of mRNA and B- 
galactosidase activity. However, because of the limitation associated with all purificationbased assays, it remains possible that the observations we made here are a result of the co-purification of contaminants. Similarly, we have gone to length to ensure limited cytoplasmic translation from the designed constructs. It is unlikely, but still possible that the observed translation might stem from cytoplasmic translation only. Consequently, further investigation is needed.

\subsubsection{Observed 6-galactosidase activity contains signatures of mitochondrial translation.}

To further study the correlation between the translation of mRNAs carrying OXA1 3'-UTR and mitochondrial translation, and to indicate that it is, in fact, mitochondrial translation which is mainly detected here and not cytoplasmic translation, we designed a control construct termed MT-C1X. This new construct is identical to MT-C1construct with the exception that its 8 nt $\operatorname{COX} 2$ translation initiation signal that promoted mitochondrial translation within $5^{\prime}$-UTR of MT-C1 was deleted. Consequently, if we were to detect cytoplasmic translation in our assays (for example, through co-purification as a contaminant) we expect relatively the same level of translation for MT-C1 and this new control construct MT-C1X. However, if we were to observe mitochondrial translation, the 6-galactosidase level observed for MT-C1X should be similar to that for MT-C2. Illustrated in Figure 4.3a, it was observed that the 6 -galactosidase activity of isolated mitochondria from strains carrying mRNAs lacking mitochondrial translation signal, MT-C1X, was reduced by approximately $90 \%$ from that observed for the intact parent construct with 
the mitochondrial translation signal (MT-C1), and to levels comparable to MT-C2 (the construct lacking OXA1 $3^{\prime}$-UTR). In this case, the level of translation associated with MTC1X may represent co-purified contaminations and the background level of translation, including cytoplasmic translation within the vicinity of mitochondria, a phenomenon that has been observed in previous studies for the natural OXA1 mRNA ${ }^{200-202}$. This observation provides evidence that 6 -galactosidase observed for MT-C1 is connected to mitochondrial translation and not as a result of co-purification artifacts.

Next, we used antibiotics that specifically targeted different modes of translation (cytoplasmic vs mitochondrial). As indicated above, yeast mitochondrial translation resembles the prokaryotic mode of translation whereas cytoplasmic translation represents that of eukaryotes. To further investigate if the B-galactosidase activity mediated by the MT-C1 construct is primarily influenced by the mitochondrial (prokaryotic) mode of translation, we utilized antibiotics thought to be specific to one mode of translation and not the other. To this end, we utilized cycloheximide and chloramphenicol. Cycloheximide is a eukaryotic translation inhibitor ${ }^{160}$. It interferes with the translation elongation step by binding to the E-site of the eukaryotic ribosome. Chloramphenicol is a bacterial translation inhibitor. It disrupts peptidyl transferase activity of the ribosome during prokaryotic translation. The presence of cycloheximide in the media reduced the level of $B$-galactosidase activity mediated by MT-C2 and MT-C1X by approximately $83 \%$ and $95 \%$, respectively (Figure $4.3 \mathrm{~b}$ ). This is in comparison with an approximately $46 \%$ reduction for MT-C1. These observations suggest that translation mediated by the MT-C1 construct seems to be more resistant to cycloheximide. In 
contrast, when the growth media was supplemented with chloramphenicol, 6galactosidase produced by MT-C2 and MT-C1X was reduced by approximately $15 \%$ and $12 \%$ indicating that they are not very sensitive to this concentration of chloramphenicol (Figure 4.3c). A reduction of $44 \%$ was observed for MT-C1 suggesting that translation mediated by MT-C1 construct appears to be more sensitive to chloramphenicol.
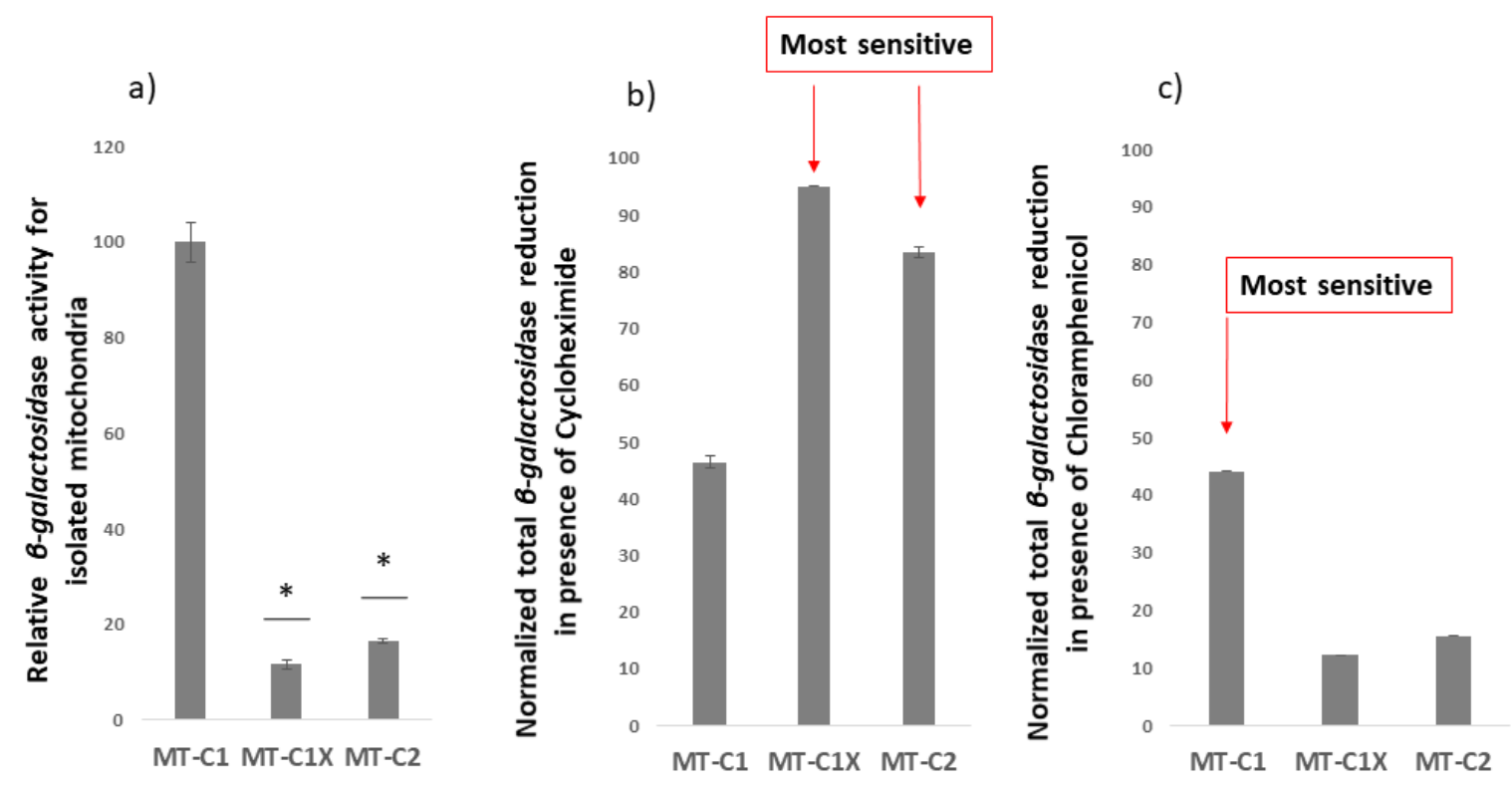

Figure 4.3. Analysis of relative 6-galactosidase activity analysis a) 6-galactosidase activity of isolated mitochondria is highly reduced in the absence of COX2 mitochondrial translation initiation signal MT-C1X in comparison to MT-C1 construct that contains the translation initiation signal. b) In the presence of $45 \mathrm{ng} / \mathrm{ml}$ of cycloheximide, a cytoplasmic translation inhibitor, 6 -galactosidase mediated by MT-C1X and MT-C2 showed the most sensitivity. c) In contrast, when $1.4 \mathrm{mg} / \mathrm{ml}$ of chloramphenicol, a prokaryotic translation inhibitor, was added 6-galactosidase activity mediated by MT-C1 showed the most sensitivity. The values in a) are related to the value of MT-C1 and values in b) and c) are normalized to the translation of the same construct in the absence of the corresponding translation inhibitory compound. ${ }^{*}$ represent statistically significant results ( $P$-value $\leq$ $0.05)$ compared to the WT. Each experiment is repeated at least 3 times. Error bars represent standard deviation. 
The observations made in the current study provide genetic evidence for a correlation between the 3'-UTR of the OXA1 gene on a reporter mRNA and yeast mitochondrial translation. In addition to the reported ability of $3^{\prime}$-UTR of OXA1 gene to direct mRNAs to the vicinity of mitochondrial, it seems possible that this $3^{\prime}$-UTR might also direct mRNAs into the mitochondria where the mRNAs can get translated by the mitochondrial translation machinery (Figure 4.4). The suggested model (Figure 4.4) should be considered at a proposal level. Detailed biochemical assays should be employed to further study this proposed model. In future, it would be of interest to further study the relationship between this $3^{\prime}$-UTR and mitochondria using other reporter genes and in other organisms including humans. The potential ability of OXA1 3'-UTR to direct an mRNA into human mitochondria could have important implications for human health. A number of human genetic diseases are linked to defects in mitochondria encoded genes or those nuclear genes whose products are directed into mitochondria ${ }^{206,207}$. In this context, the ability to direct mRNA into mitochondria could provide an appealing therapeutic opportunity. 


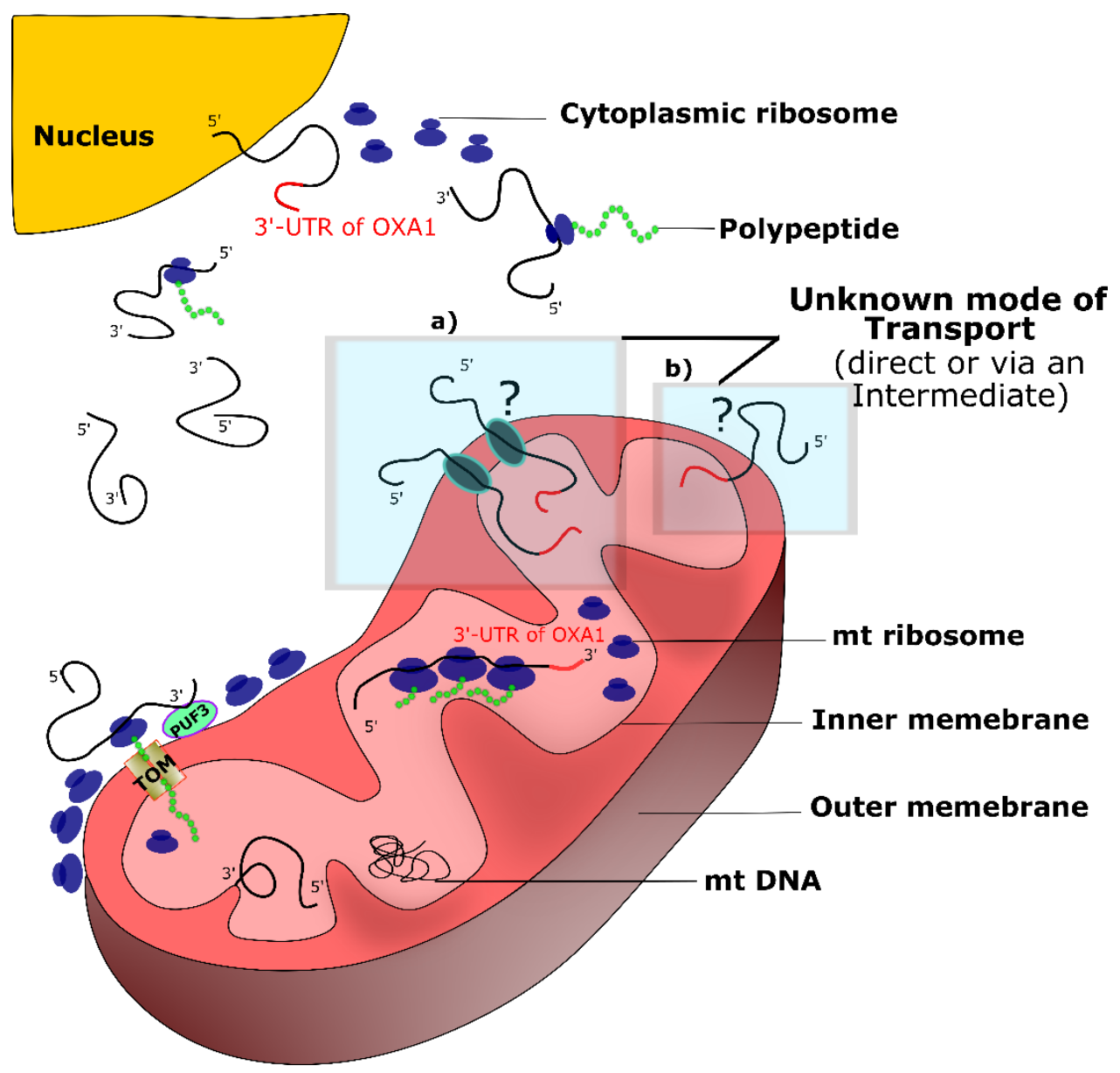

Figure 4.4. A model for the role of $3^{\prime}$-UTR of the OXA1 gene. In addition to its reported activity in recruiting mRNAs to the vicinity of mitochondria, 3'-UTR of the OXA1 gene may also direct mRNAs into mitochondria via a currently unknown mechanism. The majority of mitochondrial proteins are of nuclear origin and are imported into mitochondria after translation. The $3^{\prime}$-UTR of yeast OXA1 mRNA can direct mRNAs to the vicinity of mitochondria via interaction with mitochondria outer surface protein Puf3. The newly translated polypeptides, near the vicinity of mitochondria, are directed into mitochondria using general translocase of the outer membrane (TOM) complex. Here we propose that the 3'-UTR of OXA1 mRNA may also mediate the entry of mRNAs into mitochondria where they are translated by mitochondrial translation machinery. Figure created using Inkscape. 


\section{Translation regulation of highly structured 5'-UTR constructs.}

\subsection{Abstract}

Helicases are important for the translation of structured mRNAs. Growing evidence suggests that certain helicases affect the expression from specific mRNA structures. To further investigate proteins factors that affect the translation of specific mRNAs with distinct structured 5'-UTRs, we designed four yeast reporter constructs. In these constructs, translation of a LacZ reporter cassette is placed under the control of four different structured mammalians 5'-UTRs. Yeast knockout collection was used to identify yeast gene knockouts that show reduced expression. This resulted in the analysis of approximately 75,000 yeast colonies for $L a c Z$ expression. In this way, we identified a number of protein factors that seem to be specific to one or more structures. Interestingly we did not identify any protein factors that were linked to the translation of all 4 structures.

\subsection{Introduction}

In mammalian cells, $D h \times 29$ and $D d \times 3$ play important roles in the translation efficiency of mRNAs with strong secondary structure $\Delta \mathrm{G}<-40 \mathrm{Kcal} / \mathrm{mol}$ or long $5^{\prime}$-UTRs; for the less structured mRNAs however, they seem to have little or no effects ${ }^{90,91}$. Generally, it is accepted that elF4A is the main RNA helicase in the translation pathway that participates in unwinding secondary structures on most mRNAs. However, growing evidence suggests 
that the scanning of more structured mRNAs requires the recruitment of specific helicase enzymes ${ }^{91,104}$. The control and regulation of additional helicases seem to be connected to specific group of mRNAs with structured 5'-UTRs mainly involved in different cellular processes including regulation of growth, proliferation, and apoptosis ${ }^{90}$. Consequently, it is important to further study additional mRNA helicases and the factors that contribute to their activity as well as their regulation and control. Molecular signals that mainly regulate translation are MAPK and mTOR signals ${ }^{6}$.

Some helicases seem to be specific to certain structures. Cell under stress regulate translation of mRNAs needed for maintaining homeostasis or apoptosis. One way to control their translation is through activation of specific helicases specific for unwinding their highly structures ${ }^{135}$. It is known that retroviruses carry highly structured region in their $5^{\prime}$-UTR RNA that are crucial for their translation. These RNA structures are found to function as PCE rather than IRESs and the translation of the mRNAs with highly structured UTRs is modulated through cap-dependent protein synthesis ${ }^{102}$. This was observed in HIV-1 replication where the retrovirus requires RNA helicase A (RHA) for proper translation in the host cell for the recognition of a conserved PCE ${ }^{102}$. DDX3, DED1 in yeast, and RNA helicase $A$ in coordination with elF4A facilitate ribosomal scanning by unwinding the secondary structure or remodeling ribosome for a better scanning ${ }^{181}$.

Similarly, it was recently shown that G-quadruplex (rG4) structures on the 5'-UTR of certain human mRNAs regulate gene expression in a post-transcriptional manner ${ }^{208}$. Helicases $D H X 36$ and $D H X 9$ were shown to affect the translation of mRNAs with rG4 signatures. 
To further study helicases and protein factors that may regulate the activity of helicases as well as to identify additional ones that might be specific to a given structure, we designed four expression constructs where a reporter mRNA is under the translational control of different mammalian hairpin structures. Yeast gene knockout collection was transformed with these constructs to screen for gene deletions that reduce the activity of the reporter $L a c Z$ cassette.

\subsection{Materials and Methods}

\subsubsection{Media and Strains}

Yeast deletion set (mating type "a", (BY4741)) was used for large-scale investigation for modified-SGA to transform the constructs into mutants and to perform lift assay. Yeast mating type " $\alpha$ " (BY7092) was used for plasmid transformation to deletion set ${ }^{51}$. DH5 $\alpha$, E.coli strain, was used to generate plasmids. Standard rich (YPD 1\% Yeast extract, 2\% Peptone, 20\% D-glucose) and Synthetic Complete (SC) media were used to grow yeast. LB (Lysogeny Broth) was used to grow E.coli. $2 \%$ agar was used for all solid media. -URA media was used as a marker for selection of plasmid in yeast and LB with Ampicillin (50 $\mathrm{mg} / \mathrm{ml})$ for selection in bacteria. Geneticin, (G418), $(200 \mathrm{mg} / \mathrm{ml})$, Canavanine $(100 \mathrm{mg} / \mathrm{ml})$ were used for selective growth media in modified-SGA technique.

\subsubsection{Plasmids}

P416 plasmid with a LacZ expression cassette and a gal promoter was used as our background plasmid for cloning and as a control. To generate reporter LacZ mRNAs under the translational control of complex RNA structures, four different fragments were cloned 
upstream of the LacZ mRNA in p416 construct using Xbal restriction site. In this way four expression constructs were designed as follows: pTAR construct contains the $5^{\prime}$-UTR of HIV-tar1 gene, known to have PCE elements detected by RNA helicase A 102 (5’GGGTTCTCTGGTTAGCCAGATCTGAGCCCGGGAGCTCTCTGGCTAGCTAGGGAACCCACTGC TTAAGCCTCAATAAAGCTTGCCTTGAGTGCTTCAAGTAGTGTGTGCC 3'), pRTN that contains the 5'-UTR of FOAP-11 gene, a conserved hairpin structure in vertebrates 164 (5’GGGATTTTTTACATCGTCTTGGTAAAGGCGTGTGACCCATAGGTTTTTTAAGATCAAACACGTCT TTACAAAGGTGATCTAAGTATCTC 3'), 5'-UTR of BCL3, a transcription activator, which has been shown to be over-expressed in $\beta$-Cell lymphoma3, probably due to this highly structured mRNA ${ }^{209}$ (5' CCGTCCCCGGCGGCCCCATGCCCCGATGCCCCCGCGGGGGCC 3') and a sequence with two hairpins (5' CTCCCCCCCCCAGAAGTAGTGTTTTTTGGAGGCCTAGG CTTTTGCAAATGACCGAGTCCGTCCCCGGCGGCGGGGGGGAT 3’').

Yeast plasmid extraction was performed using yeast plasmid miniprep kit (Omega Biotek $^{\circledast}$ ) and E. coli plasmid extraction was carried out using GeneJET plasmid miniprep kit (Thermofisher ${ }^{\circledR}$ and Bio-Basics ${ }^{\circledR}$ ) according to the manufacturers' instructions.

\subsubsection{DNA transformation}

Plasmid transformation to yeast was performed using the Lithium Acetate method ${ }^{210}$ and through competent cells described by Inoue et al, ${ }^{211}$. Modified SGA technique was used to systematically transform constructs into yeast deletion library ${ }^{212}$. 


\subsubsection{6-galactosidase Assay}

White/Blue Assay or Lift Assay was performed using the X-gal compound to determine the translation activity of $L a c Z$ reporter gene ${ }^{213}$. The quantitative 6 -galactosidase assay was performed using ONPG (O-nitrophenyl- $\alpha$-D-galactopyranoside) ${ }^{32,158}$.

\subsection{Results and Discussion}

To investigate candidate genes involved in translation regulation of mRNAs with highly structured 5'-UTR, cloned plasmids pTAR, pRTN, pB-cell, and p2hair, containing 5'-UTR of HIV1-tar gene, 5'-UTR of FOAP-11 gene, 5'-UTR of BCL3 and with 2 hairpins respectively, along with p416 (as a control structure with no inhibitory structure) were systematically transformed into yeast gene deletion mutant array. This was made possible through a modified SGA technique originally designed to study Gls ${ }^{51,212}$. Plasmids were first transformed into " $\alpha$ " mating type strain. The transformed strains were then systematically mated to deletion library (nearly 5000 mutants) of "a" mating type. Diploid strains were then subjected to different rounds of selection to select haploid mutants carrying the plasmid with mutants in "a" mating type. This resulted in a total of 75,000 transformations.

To determine the translation activity of LacZ cassette in different mutants carrying target constructs we performed a B-galactosidase Lift Assay. In this assay, if the reporter gene is expressed and efficiently translated, then cells would turn blue after the addition of an X-gal solution (Figure 5.1). White colonies represent mutant strains that do not efficiently translate the structured mRNAs and hence represent potential hits. Potential hits are 
considered hits only if they were capable of producing 6 -galactosidase with the control construct (p416). In this manner, hits can in fact express the 6 -galactosidase gene but only if it does not carry an inhibitory structure. Lift assays were repeated three times for each plasmid and hits observed at least two times were selected.

In our experiments, it is expected that the deletion of genes that are associated with detecting and unwinding the hairpins would show less translation activity. Or to identify pathways that are involved in regulation of highly structured mRNAs that when mutated, translation control is inhibited. These data, combined with our data from the original construct with inhibitory structure (p281-4) that was used in previous chapters can illuminate some information about candidate genes responsible for resolving the secondary structure of these hairpins. Interestingly, there was no common factor that seems to influence the translation of all their constructs highlighting the diversity of requirements for different mRNAs. In light of our current investigation, it seems that different mRNA structures may have different protein factors required for proper translation. 


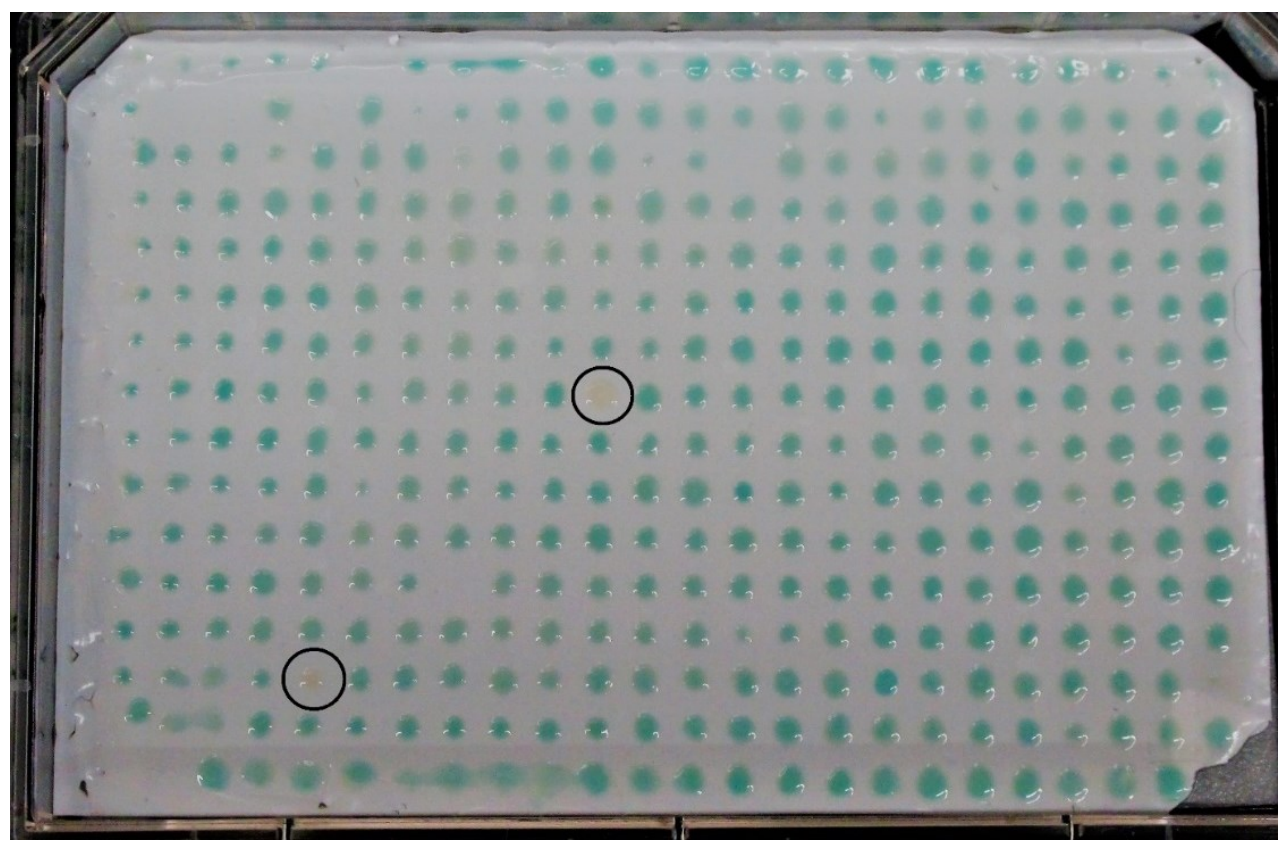

Figure 5.1. An array of deletion set, showing yeast mutant strains carrying a plasmid with a LacZ cassette reporter gene. The strains were lifted on a nitrocellulose paper, burst in liquid nitrogen and treated with $2 \% \mathrm{X}$-gal. Production of 8 -galactosidase was visible by the presence of a blue color. White colonies (shown with black circles) are those that did not produce 6 -galactosidase. They represent gene deletions where, presumably, the hairpin structures inhibited the translation of 8 -galactosidase mRNA.

To quantify translation efficiency and confirm the hits from the previous step, the liquid B-galactosidase assay was performed using the ONPG approach ${ }^{158}$ in triplicates. To this point, 118 candidate genes in a total from our four constructs were selected to be analyzed and investigated further. Table 5.1 shows the candidate genes for each plasmid. A number of candidates were found to be common to "two hairpin" structures. For example, there are four common genes between pTAR and p2hair. With a P-value of 1.2 e-5 is seems that this overlap may not be by chance alone. Consequently, there might be a statistically significant overlap between the requirements for certain hairpins. Further 
analysis is required to establish a relationship between these protein factors and the structure of the hairpins.

Our preliminary GO term enrichment analysis of the hits revealed that many of the hits are involved in the regulation of signaling pathways, negative regulation of the nucleobases-containing compound metabolic process, regulation of cell communication and carbohydrate kinase activity. Considering the fact that mRNA helicases actually are involved in most processes related to mRNA metabolism including transcription, splicing, mRNA export, decay and other pathways like ribosome biogenesis and translation we can propose that network analysis of our candidate genes represent a broad range of these pathways specially in translation control and signaling pathways under stress.

At this point, we have finalized the analysis of approximately 75,000 yeast colonies and generated preliminary screening data. Additional complimentary experiments are required to narrow down possible candidate genes involved in unwinding these structures. For example, although our experience has shown that most of the hits generated in this fashion identify genes that target translation, it remains a possibility that many of them may influence other processes including transcription and mRNA stability. 
Table 5-1. Candidate genes that seem to influence the expression of different 6 galactosidase constructs pTAR, pRTN, pB-cell and p2hair. Selected hits were chosen based on the white color performing lift assay (repeated three times and was observed at least twice in the repeats) and confirmed by liquid 6 -galactosidase assay. The red cells are representing common genes between different constructs and yellow cells represent those that were identified in our previous screenings.

\begin{tabular}{|c|c|c|c|}
\hline pTAR & pRTN & pBcell & p2hair \\
\hline MFT1 & GAL3 & MFT1 & FRT1 \\
\hline PEX7 & RTT106 & FRT1 & GAL3 \\
\hline RRN10 & URA2 & SEC22 & MFT1 \\
\hline THP2 & GUT2 & YNL140C & PEX7 \\
\hline YNL140C & IML3 & IRC3 & RRN10 \\
\hline PUB1 & MRX1 & HSP31 & RTT106 \\
\hline PRM2 & PHO87 & KEX2 & SEC22 \\
\hline PPM1 & PPR1 & LSB3 & THP2 \\
\hline RPP1A & REV7 & GPD2 & URA2 \\
\hline SCS3 & RPL23A & NCA2 & YNL140C \\
\hline SKI2 & RRD1 & NVJ2 & NAM7 \\
\hline URA4 & CSN12 & PEX31 & PUS2 \\
\hline YMR226C & SAP4 & PEX9 & SET6 \\
\hline YOR072W & $\mathrm{SBH} 2$ & PIR3 & NSI1 \\
\hline CMP2 & Ski5 & PPG1 & CSE2 \\
\hline HSL1 & SLI15 & PTC1 & FLX1 \\
\hline \multirow[t]{16}{*}{ MET18 } & SMI1 & RGT1 & FUN9 \\
\hline & SWH1 & RPS6A & RSC2 \\
\hline & TAE1 & HNM1 & GIM3 \\
\hline & CEM1 & SIF2 & SAC7 \\
\hline & YBR042C & SKO1 & HEF3 \\
\hline & YIL067C & STC2 & SEM1 \\
\hline & YJL045W & TDH3 & CIN5 \\
\hline & YJL163C & WTM1 & SKS1 \\
\hline & YJR084W & YCL042W & SWI4 \\
\hline & YLR114C & YDR210W & SWI6 \\
\hline & YRO2 & YKL133C & TEP1 \\
\hline & & YLR404W & LSM6 \\
\hline & & YMR018W & TOP1 \\
\hline & & FET3 & MIX14 \\
\hline & & YPR091C & YDR338C \\
\hline & & & YMR289W \\
\hline
\end{tabular}




\begin{tabular}{|l|l|} 
& YNL003C \\
YNL128W \\
MRN1 \\
YNL153C \\
YNL171C \\
YPL034W \\
YPL184C \\
\hline
\end{tabular}




\section{Conclusion}

\subsection{Concluding Remarks}

One of the main objectives of our research laboratory is to investigate the novel function of genes in various pathways. This is often done using systems biology approaches and through large-scale screening followed by complementary experiments. Discovering novel functions for genes, complexes and pathways can lead to a broader understanding of how a cell functions as a living system. For our investigations, we focus on the baker's yeast, $S$. cerevisiae. As a tool, this model organism not only facilities high throughput experiments developed for molecular genetic studies, but also serves as an asset because of its considerable homology with the human genome and with genes causing certain complex diseases in humans. With the availability of different arrays including yeast mutant array of nearly 5000 non-essential genes, over-expression array, and GFP-tagged yeast ORF library along with different bioinformatics software for analyzing, visualizing and extracting meaningful information from large-scale data, yeast has become a good candidate for functional genomics studies $8,24,54$.

Translation pathway, a conserved pathway in eukaryotes and prokaryotes, is responsible for the production of functional and structural proteins in the cell. Proteins are considered as structural and transportation molecules in the cell along with their enzymatic activities which are all crucial for cell survival and cell maintenance. Malfunction or dysregulation in this pathway can cause cell death and give rises to different types of complex diseases in humans. Diseases like cancer, neurodegenerative diseases and cognitive disorders have 
been linked to failure in proper function of translation or in translation regulation processes. Hence, this pathway has been the target of therapeutic researches for a long time. Translation initiation is often considered as the most rate-limiting step in gene expression and is generally regulated through the cooperation of numerous eukaryotic initiation factors, mRNA properties, and ribosomes. Mutations in initiation factors have been linked to important diseases. For example, it is observed that in B-cell lymphoma cells, translation regulation becomes defective and causes higher translation rate of oncogenic genes specifically the ones with highly structured mRNAs ${ }^{209}$ proposing to be due to over-expression of elF4B an activator of elF4A, which causes a higher rate of translation for oncogenic genes. Another example is in HIV infection where the virus uses the host translation machinery to specifically regulate its own genome. The HIV virus genome consists of mRNAs with highly structured 5'-UTR detectable by specific RNA helicases like RNA helicase $A^{102}$.

RNA helicases are responsible for unwinding secondary structures of mRNA and in some cases remodeling of the $40 \mathrm{~S}$ ribosomal subunit for better scanning of mRNA through the translation process. They also play roles in transcription, mRNA splicing, mRNA export and storage, translation activation/inhibition and mRNA decay pathway ${ }^{98,214,215}$. In addition to helicase activity RNA helicases may have other biochemical activities including RNA clamping, double-stranded RNA destabilization, dissociation of proteins from RNAs and strand annealing ${ }^{216}$. elF4A, the main known helicase of translation pathway, is a component of elF4F complex and is known to regulate translation of most mRNAs in the cell and is responsible for resolving 5' cap structure, whereas other RNA helicases are 
thought to be involved in recognition and unwinding of mRNAs with highly structured 5'UTRs like DED1 and its close homolog DBP1 in human. It is proposed that different types of mRNAs, based on their secondary structure at 5'-UTR, demand different types of helicases and associated factors to regulate their translation ${ }^{217}$.

The structure of mRNA $5^{\prime}$-UTR is a feature that can regulate the post-transcriptional expression of different mRNAs. This regulation, at the translation level, can be in a capdependent or cap-independent manner. In cap-dependent regulation, usually remolding or unwinding of RNA structures happens with the help of different RNA helicases or other RNA interaction processes (for example with sRNAs) to regulate scanning. In a capindependent manner, the regulation is through direct recognition of RNA structures like IRES or PCE elements and mRNA modifications ${ }^{217}$. New findings show that the structure of mRNA, regardless of its negative folding free energy, can recruit translation machinery even more efficiently than those with no hairpin structure ${ }^{217}$.

Although much has been learned about the function of known RNA helicase involved in translation and other pathways, there is still much to learn about the novel function of different factors that can influence the translation of structured mRNAs. mRNA structure is one of the components that determine the rates and regulation of mRNA translation. In a recent study using ribosome profiling under inhibition of elF4A by different drugs like Silvestrol and Rocalglamide, it was observed that the translation of mRNAs with CGG motifs was selectively reduced but not mRNAs with long UTRs ${ }^{217}$. Another study that investigated 5'-UTR of mRNAs through deletion of elF4A followed by ribosome profiling 
and RNA sequencing revealed U-rich and GA-rich motifs on mRNAs as important regulators of translation 218,219 .

In the current thesis, we investigated novel yeast gene functions that affect the translation of structured mRNAs. For this, we screen the library of yeast nonessential gene deletion for those genes that affect the translation of a structured reporter construct.

Using our original structured reporter construct p281-4, 24 candidate genes were

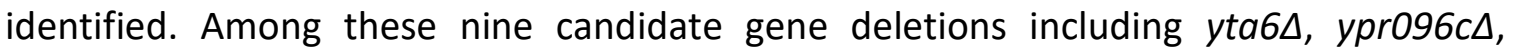
nam $7 \Delta$, pus $2 \Delta, r p / 27 b \Delta, r p s 23 b \Delta, \operatorname{srs} 2 \Delta, y p r 089 w \Delta$, and hal1 $\Delta$ showed the most sensitivity to $\mathrm{LiCl}$ treatment $(10 \mathrm{mM})$. Further, we showed that over-expression of the mentioned genes can revert $\mathrm{LiCl}$ toxicity in the cell, in the same that over-expression of elF4A compensated $\mathrm{LiCl}$ sensitivity ${ }^{148}$. Follow up experiments performed to confirm that this was due to translation deficiency rather than reduction at the transcription level.

In the next phase of our investigation, to further study different protein factors required for the translation of specific structured mRNAs we designed additional. Four constructs named pTAR, pRTN, pB-cell, and p2hair were generated for this purpose. The early screening revealed some mutual hits with our previous data, including NAM7, PUS2, IRC3, SET6, and PUB1. Certain overlaps were observed between protein requirements for different structures. However, no common protein factor was found in all the screens. This further highlights that protein requirements for translation of different mRNAs are in fact variable. Further study is required to confirm our preliminary results. We expect most of these genes to indirectly affect the translation. Consequently, future studies can 
focus on generating double gene deletions to illustrate the intermediator gene(s) or pathway(s) that are influenced by these genes. Some of these genes may also have helicase activities. In this context in vitro assays designed to study the activity of helicases will prove to be useful.

In another study, we used translation in mitochondria as a strategy to investigate the ability of a specific 3 '-UTR sequence to direct a reporter mRNA into yeast mitochondria. Our constructs were designed based on 3'-UTR sequence of OXA1, known to direct its mRNA to the vicinity of mitochondria. Previous studies show that Oxa1p is produced in the vicinity of mitochondria and then transferred into mitochondria ${ }^{190}$. Here we aimed to see if some of these mRNAs can get into mitochondria directly. Our observations provided genetic evidence for the import of a reporter 6-galactosidase mRNA into mitochondria that are mediated by the $3^{\prime}$-UTR of the OXA1 gene. Malfunction of different mitochondrial proteins is linked to various diseases. Consequently, the ability of an RNA sequence to direct mRNA into mitochondria might have therapeutic uses.

In this thesis, we investigated the activity of five genes for their novel involvement in the translation of structured mRNAs. We also performed a directed large scale screening for similar gene function and in doing so observed dozens of new functions for previously investigated genes. A conclusion that we can draw from these observations is that there seems to be much to be learned for the translation of specific mRNAs mediated by specific structures. It now seems that these structures might play more important roles than previously thought. Similarly, the involvement of additional genes in translation regulation also suggests a higher degree of complexity for translation regulation mediated 
by mRNA structure. In this thesis, we only focused on certain structures. Expanding the diversity of these structures may help us discover additional gene functions related to the translation of structured mRNAs. In this context, it is worth noting that by no means are the screens we performed exhaustive. Consequently, there remains a number of additional factors that might be identified using additional and improved screens. For example, our screens only focused on non-essential genes. Including conditional knockout essential gene array into future screens can provide additional information. Similarly, our current screens are based on negative selection where the deletion of a target gene reduces the ability of translation from a structured mRNA. Future screens can focus on positive selections were the over-expression of a target gene may increase the translation of a structured mRNA.

The discovery of so many new gene functions in the current thesis also highlights the need for additional functional genomics studies. The current screen revealed dozens of potential new gene functions related to one specific feature of translation. We expect that the development of new functional genomics screens with different areas of focus can mediate the identification of additional gene functions for other aspects of translation and translation control as well as other cellular processes. The discovery of additional functions for genes can help us better realize the biology of a cell as a system and its mechanism of responses to internal and external stimuli. 


\section{References}

1. Covert MW. Fundamentals of Systems Biology: From Synthetic Circuits to WholeCell Models. (Group T\& F, ed.). CRC Press; 2015.

http://www.taylorandfrancis.com.

2. Edda K, Wolfram, Liebermeister Christoph W, Axel K. Systems Biology: A Textbook. John Wiley \& Sons; 2016.

3. Ebhardt HA, Root A, Sander C, Aebersold R. Applications of targeted proteomics in systems biology and translational medicine. Proteomics. 2015;15(18):31933208. doi:10.1002/pmic.201500004

4. Kitano H. Systems Biology : A Brief Overview. Science (80- ). 2002;295(5560):1662-1665. doi:10.1126/science.1069492

5. Kirschner MW. The Meaning of Systems Biology. Cell. 2005;121(4):503-504. doi:10.1016/j.cell.2005.05.005

6. Kim H. Cell Fate Control by Translation : mRNA Translation Initiation as a Therapeutic Target for Cancer Development and Stem Cell Fate Control. Biomolecules. 2019;9(665). doi:https://doi.org/10.3390/biom9110665

7. Boone C, Bussey $\mathrm{H}$, Andrews BJ. Exploring genetic interactions and networks with yeast. Nat Rev Genet. 2007;8(6):437-449. doi:10.1038/nrg2085

8. Pitre S, Dehne F, Chan A, et al. PIPE : a protein-protein interaction prediction engine based on the re-occurring short polypeptide sequences between known 
interacting protein pairs. BMC Bioinformatics. 2006;15:1-15. doi:10.1186/14712105-7-365

9. Schoenrock A, Samanfar B, Pitre S, et al. Efficient prediction of human proteinprotein interactions at a global scale. BMC Bioinformatics. 2014:1-22. doi:10.1186/s12859-014-0383-1

10. Babu M, Arnold R, Bundalovic-torma C, et al. Quantitative Genome-Wide Genetic Interaction Screens Reveal Global Epistatic Relationships of Protein Complexes in Escherichia coli. PLOS. 2014;10(2). doi:10.1371/journal.pgen.1004120

11. Pitre S, North C, Alamgir M, et al. Global investigation of protein - protein interactions in yeast Saccharomyces cerevisiae using re-occurring short polypeptide sequences. Nucleic Acids Res. 2008;36(13):4286-4294. doi:10.1093/nar/gkn390

12. Amos-binks A, Patulea C, Pitre S, et al. Binding Site Prediction for Protein-Protein Interactions and Novel Motif Discovery using Re-occurring Polypeptide Sequences. BMC Bioinformatics. 2011;12(1):225. doi:10.1186/1471-2105-12-225

13. Jessulat $M$, Pitre $S$, Gui $Y$, et al. Recent advances in protein-protein interaction prediction: experimental and computational methods. Expert Opin Drug Discov. 2011;6(9):921-935. doi:10.1517/17460441.2011.603722

14. Tokheim CJ, Papadopoulos N, Kinzler KW, Vogelstein B, Karchin R. Evaluating the evaluation of cancer driver genes. PNAS. 2016;113(50). 
doi:10.1073/pnas.1616440113

15. Stynen B, Tavernier J, Dijck P Van. Diversity in Genetic In Vivo Methods for Protein-Protein Interaction Studies : from the Yeast Two-Hybrid System to the Mammalian Split-Luciferase system. Microbiol Mol Biol Rev. 2012;76(2):331-382. doi:10.1128/MMBR.05021-11

16. Brückner A, Polge C, Lentze N, Auerbach D, Schlattner U. Yeast Two-Hybrid, a Powerful Tool for Systems Biology. Mol Sci. 2009:2763-2788. doi:10.3390/ijms10062763

17. Zhu H, Snyder M. Protein chip technology. Curr Opin Chem Biol. 2003:55-63. doi:10.1016/S1367-5931(02)00005-4

18. Schuldiner M, Collins SR, Thompson NJ, et al. Exploration of the Function and Organization of the Yeast Early Secretory Pathway through an Epistatic Miniarray Profile. Cell. 2005;123:507-519. doi:10.1016/j.cell.2005.08.031

19. Ho Y, Gruhler A, Heilbut A, et al. Systematic identification of protein complexes in Saccharomyces cerevisiae by mass spectrometry. Lett to Nat. 2002;415(January):2-5. doi:https://doi.org/10.1038/415180a

20. Ong Q, Nguyen P, Thao NP, Le L. Bioinformatics Approach in Plant Genomic Research. Curr Genomics. 2016:368-378.

doi:10.2174/1389202917666160331202956

21. Mangul S, Martin LS, Hill BL, et al. Systematic benchmarking of omics 
computational tools. Nat Commun. 2019;(2019):1-11. doi:10.1038/s41467-01909406-4

22. Droit A, Poirier GG, Hunter JM. Experimental and bioinformatic approaches for interrogating protein - protein interactions to determine protein function. $J \mathrm{Mol}$ Endocrinol. 2005;34:263-280. doi:10.1677/jme.1.01693

23. Gehlenborg N, Donoghue SIO, Baliga NS, et al. Visualization of omics data for systems biology. Nat Publ Gr. 2010;7(3s):S56-S68. doi:10.1038/nmeth.1436

24. Wagih O, Usaj M, Baryshnikova A, et al. SGAtools : one-stop analysis and visualization of array-based genetic interaction screens. Nucleic Acids Res. 2013;41(May):591-596. doi:10.1093/nar/gkt400

25. Pavlopoulos GA, Malliarakis D, Papanikolaou N, Theodosiou T, Enright AJ, Iliopoulos I. Visualizing genome and systems biology : technologies, tools, implementation techniques and trends, past, present and future. Gigascience. 2015;4(38). doi:10.1186/s13742-015-0077-2

26. Bunnik EM, Roch KG Le. An Introduction to Functional Genomics and Systems Biology. Adv Wound Care. 2013;2(9):490-498. doi:10.1089/wound.2012.0379

27. Omidi K, Jessulat M, Hooshyar M, et al. Uncharacterized ORF HUR1 influences the efficiency of non-homologous end-joining repair in Saccharomyces cerevisiae. Gene. 2018;639. doi:10.1016/j.gene.2017.10.003

28. Omidi K, Hooshyar M, Jessulat M, et al. Phosphatase Complex Pph3 / Psy2 Is 
Involved in Regulation of Efficient Non-Homologous End-Joining Pathway in the Yeast Saccharomyces cerevisiae. Plose one. 2014;9(1).

doi:10.1371/journal.pone.0087248

29. Jessulat M, Malty RH, Nguyen-tran D, et al. Spindle Checkpoint Factors Bub1 and Bub2 Promote DNA DoubleStrand Break Repair by Nonhomologous End Joining. mcb. 2015;35(14):2448-2463. doi:10.1128/MCB.00007-15

30. Samanfar B, Omidi K, Hooshyar M, et al. Large-scale investigation of oxygen response mutants in Saccharomyces cerevisiae. Mol Biosyst. 2013;9(6):13511359. doi:10.1039/c3mb25516f

31. Moteshareie $\mathrm{H}$, Hajikarimlou M, Indrayanti AM, et al. Heavy metal sensitivities of gene deletion strains for ITT1 and RPS1A connect their activities to the expression of URE2, a key gene involved in metal detoxification in yeast. Plose one. 2018;13:1-18. doi:10.1371/journal.pone.0198704

32. Krogan NJ, Kim M, Tong A, et al. Methylation of Histone H3 by Set2 in Saccharomyces cerevisiae Is Linked to Transcriptional Elongation by RNA Polymerase II. mcb. 2003;23(12):4207-4218. doi:10.1128/mcb.23.12.42074218.2003

33. Alamgir M, Jessulat M, Azizi A, Golshani A. Chemical-genetic profile analysis of five inhibitory compounds in yeast. bmc Chem Biol. 2010;10(6). doi:10.1186/1472-6769-10-6 
34. Samanfar B, Shostak K, Moteshareie H, et al. The sensitivity of the yeast, Saccharomyces cerevisiae, to acetic acid is influenced by DOM34 and RPL36A. PeerJ. 2017;2017(11). doi:10.7717/peerj.4037

35. Scannell DR, Zill OA, Rokas A, et al. The awesome power of yeast evolutionary genetics: New genome sequences and strain resources for the Saccharomyces sensu stricto genus. G3 Genes, Genomes, Genet. 2011;1(1):11-25. doi:10.1534/g3.111.000273

36. Suter B, Auerbach D, Stagljar I. Yeast-based functional genomics and proteomics technologies: The first 15 years and beyond. Biotechniques. 2006;40(5):625-644. doi:10.2144/000112151

37. Mercatanti A, Lodovichi S, Cervelli T, Galli A. CRIMEtoYHU : a new web tool to develop yeast-based functional assays for characterizing cancer-associated missense variants. FEMS. 2017;(September):1-11. doi:10.1093/femsyr/fox078

38. Yang F, Sun S, Tan G, et al. Identifying pathogenicity of human variants via paralog-based yeast complementation. PLoS Genet. 2017:1-21. doi:10.1371/journal.pgen.1006779

39. Kachroo A., Laurent J., Yellman C., Meyer A., Wike C., Marcotte E. Systematic humanization of yeast genes reveals consesrved functions and genetic modularity. Science (80- ). 2015;348(6237):921-926. doi:10.1126/science.aaa0769

40. Aouida M, Ramotar D. The Budding Yeast Saccharomyces cerevisiae as A Model 
System for Anti-Cancer Drug Screening. Clin Oncol. 2018;1(6):6-7.

https://clinicsofoncology.com/pdf/COO-v1-1025.pdf.

41. Balibar CJ, Roemer T. Yeast : a microbe with macro-implications to antimicrobial drug discovery Double Knockout. Brief Funct Genomics. 2016;15(October 2015):147-154. doi:10.1093/bfgp/elv038

42. Yu R, Nielsen J. Yeast systems biology in understanding principles of physiology underlying complex human diseases. Curr Opin Biotechnol. 2020;63:63-69. doi:10.1016/j.copbio.2019.11.021

43. Pitre S, Hooshyar M, Schoenrock A, et al. Short co-occurring polypeptide regions can predict global protein interaction maps. Sci Rep. 2012;2:1-10. doi:10.1038/srep00239

44. Tong AH, Lesage $\mathrm{G}$, Bader $\mathrm{GD}$, et al. Global mapping of the yeast genetic interaction network. Science (80- ). 2004;303(5659):808-813. doi:10.1126/science.1091317

45. Pan X, Yuan DS, Xiang D, et al. A Robust Toolkit for Functional Profiling of the Yeast Genome. Mol Cell. 2004;16:487-496. doi:10.1016/j.molcel.2004.09.035

46. Jessulat M, Alamgir M, Salsali H, Greenblatt J, Jianhua X, Golshani A. Interacting proteins Rtt109 and Vps75 affect the efficiency of non-homologous end-joining in Saccharomyces cerevisiae. Arch Biochem Biophys. 2008;469:157-164.

doi:10.1016/j.abb.2007.11.001 
47. Butland G, Krogan NJ, Xu J, et al. Investigating the in vivo activity of the DeaD protein using protein-protein interactions and the translational activity of structured chloramphenicol acetyltransferase mRNAs. J Cell Biochem. 2007;100(3):642-652. doi:10.1002/jcb.21016

48. Babu M, Cedoljub B-T, Charles C, et al. Global landscape of cell envelope protein complexes in Escherichia coli. Nat Biotechnol. 2018;36(1):103-112. doi:10.1038/nbt.4024.Global

49. Goffeau A, Barrell B, Bussey H. Life with 6000 genes. Science (80- ). 1996;274(5287):546-567. doi:10.1126/science.274.5287.546

50. Giaever G, Nislow C. The Yeast Deletion Collection : A Decade of Functional Genomics. Genetics. 2014;197(June):451-465. doi:10.1534/genetics.114.161620

51. Tong A, Evangelista M, Parsons AB, et al. Systematic Genetic Analysis with Ordered Arrays of Yeast Deletion Mutants. Science (80- ). 2001;294(December):2364-2369. doi:10.1126/science.1065810

52. Mani R, St.Onge RP, Hartman JL, Giaever G, Roth FP. Defining genetic interaction. Proc Natl Acad Sci. 2008;105(9):3461-3466. doi:10.1073/pnas.0712255105

53. Baryshnikova A, Costanzo M, Dixon S, et al. Synthetic Genetic Array ( SGA) Analysis in Saccharomyces Cerevisiae and Schizosaccharomyces Pombe. Vol 470. 2nd ed. Elsevier Inc.; 2010. doi:10.1016/S0076-6879(10)70007-0

54. Memarian $\mathrm{N}$, Jessulat $\mathrm{M}$, Alirezaie J, et al. Colony size measurement of the yeast 
gene deletion strains for functional genomics. BMC Bioinformatics. 2007;8(117). doi:10.1186/1471-2105-8-117

55. Wetherow O, Green J, Chan ADC, Golshani A. Plate analyzer - A yeast colony size measurement system. IEEE. 2010:140-144. doi:10.1109/MEMEA.2010.5480211

56. Leeuwen van J, Boone C, Andrews BJ. Mapping a diversity of genetic interactions in yeast. Curr Opin Syst Biol. 2017;6:14-21. doi:10.1016/j.coisb.2017.08.002

57. Omidi K, Jessulat $\mathrm{M}$, Hooshyar M, et al. Uncharacterized ORF HUR1 influences the efficiency of non-homologous end-joining repair in Saccharomyces cerevisiae. Gene. 2018;639:128-136. doi:10.1016/j.gene.2017.10.003

58. Parsons $A B$, Brost $R L$, Ding $H$, et al. Integration of chemical-genetic and genetic interaction data links bioactive compounds to cellular target pathways. Nat Biotechnol. 2004;22(1):62-69. doi:10.1038/nbt919

59. Burnside D, Houman M, Marquez I, et al. Bioactive Natural Products: Chemistry and Biology. In: Brahmachari G, ed. Wiley online library; 2014. doi:https://doi.org/10.1002/9783527684403.ch2

60. Galván Márquez I, Akuaku J, Cruz I, Cheetham J, Golshani A, Smith ML. Disruption of protein synthesis as antifungal mode of action by chitosan. Int J Food Microbiol. 2013;164(1):108-112. doi:10.1016/j.ijfoodmicro.2013.03.025

61. Mir-Rashed N, Cruz I, Jessulat M, et al. Disruption of fungal cell wall by antifungal Echinacea extracts. Med Mycol. 2010;48(7):949-958. 
doi:10.3109/13693781003767584

62. Márquez IG, Ghiyasvand M, Massarsky A, et al. Zinc oxide and silver nanoparticles toxicity in the baker's yeast, Saccharomyces cerevisiae. PLoS One. 2018;13(3):119. doi:10.1371/journal.pone.0193111

63. Darvishi E, Omidi M, Bushehri AAS, Golshani A, Smith ML. The Antifungal Eugenol Perturbs Dual Aromatic and Branched-Chain Amino Acid Permeases in the Cytoplasmic Membrane of Yeast. PLoS One. 2013;8(10).

doi:10.1371/journal.pone.0076028

64. Galván IJ, Mir-Rashed N, Jessulat M, et al. Antifungal and antioxidant activities of the phytomedicine pipsissewa, Chimaphila umbellata. Phytochemistry. 2008;69(3):738-746. doi:10.1016/j.phytochem.2007.09.007

65. Darvishi E, Omidi M, Bushehri AA, Golshani A, Smith ML. Thymol antifungal mode of action involves telomerase inhibition. Med Mycol. 2013;51(8):826-834. doi:10.3109/13693786.2013.795664

66. Bandyopadhyay S, Mehta M, Kuo D, et al. Rewiring of Genetic Networks in Response to DNA Damage. Science (80- ). 2010;330(6009):1385-1389. doi:10.1126/science.1195618.Rewiring

67. Hershey JWB, Sonenberg N, Mathews MB. Principles of Translational Control : An Overview. Cold Spring Harb Perspect Biol. 2012.

doi:10.1101/cshperspect.a011528 
68. Mathews MB, Sonenberg N, Hershey JWB. Translatoinal Control in Biology and Medicine. illustrate. CSHL Press; 2007. https://www.cshlpress.com/link/tranbio.htm.

69. Taylor JD, Devkota B, Huang A, et al. Comprehensive Molecular Structure of the Eukaryotic Ribosome. Structure. 2009;23(1):1-7. doi:10.1038/jid.2014.371

70. Roux PP, Topisirovic I. Regulation of mRNA Translation by Signaling Pathways. Cold Spring Harb Perspect Biol. 2012;4(11):1-23.

doi:10.1101/cshperspect.a012252

71. Lafontaine DLJ, Tollervey D. THE FUNCTION AND SYNTHESIS OF RIBOSOMES. Nat Rev Mol Cell Biol. 2001;2(July):514-520. doi:10.1038/35080045

72. Wilson DN, Cate JHD. The Structure and Function of the Eukaryotic Ribosome. Cold Spring Harb Perspect Biol. 2012:1-17. doi:10.1101/cshperspect.a011536

73. Kozak M. Regulation of translation via mRNA structure in prokaryotes and eukaryotes. Gene. 2005;361(1-2):13-37. doi:10.1016/j.gene.2005.06.037

74. Shirokikh NE, Spirin AS. Poly ( A ) leader of eukaryotic mRNA bypasses the dependence of translation on initiation factors. PNAS. 2008;105(31):1073810743. doi:10.1073/pnas.0804940105

75. Mitchell S., Walker S., Algire M., Park E, Hinnebusch AG, Lorsch J. The 5'-7methylguanosine cap on eukaryotic mRNAs serves both to stimulate canonical translation initiation and block an alternative pathway. Mol Cell. 2011;39(6):950- 
962. doi:10.1016/j.molcel.2010.08.021.The

76. Hinnebusch AG, Lorsch JR. The Mechanism of Eukaryotic Translation Initiation : New Insights and Challenges. Cold Spring Harb Perspect Biol. 2012:1-25. doi:10.1101/cshperspect.a011544.

77. Dmitriev SE, Terenin IM, Andreev DE, et al. GTP-independent tRNA Delivery to the Ribosomal P-site by a Novel Eukaryotic Translation Factor. Biol Chem. 2010;285(35):26779-26787. doi:10.1074/jbc.M110.119693

78. Henderson A, Hershey JWB, Henderson A, Hershey JWB. The role of elF5A in protein synthesis The role of elF5A in protein synthesis a $\mathrm{n} \mathrm{d} \mathrm{e.} \mathrm{Cell} \mathrm{Cycle.}$ 2011;4101:20-22. doi:10.4161/cc.10.21.17850

79. Hui L, Yi-fei Z, Juan X, Rong W, Zhengping J. Potential extra-ribosomal functions of ribosomal proteins in Saccharomyces cerevisiae. Microbiol Res. 2015;177:28-33. doi:10.1016/j.micres.2015.05.004

80. Bush MS, Pierrat O, Nibau C, et al. elF4A RNA Helicase Associates with CyclinDependent Protein Kinase A in Proliferating Cells and Is Modulated by Phosphorylation. Plant Physiol. 2016;172(September):128-140. doi:10.1104/pp.16.00435

81. Gagarinova A, Stewart G, Samanfar B, et al. Systematic Genetic Screens Reveal the Dynamic Global Functional Organization of the Bacterial Translation Machinery. Cell Rep. 2016;17(3):904-916. doi:10.1016/j.celrep.2016.09.040 
82. Vlasblom J, Zuberi K, Rodriguez $\mathrm{H}$, et al. Novel function discovery with GeneMANIA: A new integrated resource for gene function prediction in Escherichia coli. Bioinformatics. 2015;31(3):306-310.

doi:10.1093/bioinformatics/btu671

83. Hu P, Janga SC, Babu M, et al. Global functional atlas of Escherichia coli encompassing previously uncharacterized proteins. PLoS Biol. 2009;7(4):09290947. doi:10.1371/journal.pbio.1000096

84. Kumar A, Beloglazova N, Bundalovic-Torma C, et al. Conditional Epistatic Interaction Maps Reveal Global Functional Rewiring of Genome Integrity Pathways in Escherichia coli. Cell Rep. 2016;14(3):648-661. doi:10.1016/j.celrep.2015.12.060

85. Ruggero D. Translational Control in Cancer Etiology. Cold Spring Harb Perspect Biol. 2013;5(2):1-27. doi:doi: 10.1101/cshperspect.a012336.

86. Silvera D, Formenti SC, Schneider RJ. Translational control in cancer. Nat Publ Gr. 2010;10(April). doi:10.1038/nrc2824

87. Bottley A, Kondrashov A. Aberrant translation of proteins implicated in Alzheimer ' s disease pathology. OA Genet. 2013;1(1):1-5. doi:10.13172/2054-197X--1-918

88. Eberle J, Krasagakis K, Orfanos C. TRANSLATION INITIATION FACTOR eIF-4A1 mRNA IS CONSISTENTLY OVEREXPRESSED IN HUMAN MELANOMA CELLS IN VITRO. Int J Cancer. 1997;401(December 1996):396-401. doi:10.1002/(sici)1097- 
0215(19970502)71:3<396::aid-ijc16>3.0.co;2-e

89. Bottley A, Phillips NM, Webb TE, Willis AE, Spriggs KA. elF4A Inhibition Allows Translational Regulation of mRNAs Encoding Proteins Involved in Alzheimer 's Disease. Plose one. 2010;5(9). doi:10.1371/journal.pone.0013030

90. Parsyan A, Shahbazian D, Martineau Y, et al. The helicase protein DHX29 promotes translation initiation, cell proliferation, and tumorigenesis. PNAS. 2009;106:22217-22222. doi:doi: 10.1073/pnas.0909773106

91. Lai M, Lee YW, Tarn W. The DEAD-Box RNA Helicase DDX3 Associates with Export Messenger Ribonucleoproteins as well as Tip-associated Protein and Participates in Translational Control. Mol Biol Cell. 2008;19(September):3847-3858. doi:10.1091/mbc.E07

92. Ozes A., Feokjtistova K, Avanzino B., Fraser C. Duplex unwinding and ATPase activities of the DEAD-box helicase elF4A are coupled by elF4G and elF4B. $\mathrm{J} \mathrm{Mol}$ Biol. 2012;412(4):674-687. doi:10.1016/j.jmb.2011.08.004.Duplex

93. Hinnebusch AG. Structural Insights into the Mechanism of Scanning and Start Codon Recognition in Eukaryotic Translation Initiation. Trends Biochem Sci. 2017;42(8):589-611. doi:10.1016/j.tibs.2017.03.004

94. Hinnebusch AG. Molecular Mechanism of Scanning and Start Codon Selection in Eukaryotes. Microbiol Mol Biol Rev. 2011;75(3):434-467. doi:10.1128/MMBR.00008-11 
95. Elfakess R, Sinvani H, Haimov O, Svitkin Y, Sonenberg N, Dikstein R. Unique translation initiation of mRNAs-containing TISU element. Nucleic Acids Res. 2011;39(17):7598-7609. doi:10.1093/nar/gkr484

96. Hartman TR, Qian S, Bolinger C, Fernandez S, Schoenberg DR, Boris-lawrie K. RNA helicase A is necessary for translation of selected messenger RNAs. Nat Struct Mol Biol. 2006;13(6):509-516. doi:10.1038/nsmb1092

97. Minshall N, Kress M, Weil D, Standart N. Role of p54 RNA Helicase Activity and Its C-terminal Domain in Translational Repression, P-body Localization and Assembly. Mol Biol Cell. 2009;20:2464-2472. doi:10.1091/mbc.E09

98. Soto-Rifo R, Rubilar PS, Limousin T, De Breyne S, D??cimo D, Ohlmann T. DEADbox protein DDX3 associates with elF4F to promote translation of selected mRNAs. EMBO J. 2012;31(18):3745-3756. doi:10.1038/emboj.2012.220

99. Keegan R, Yan W. Control of Messenger RNA Fate by RNA-Binding Proteins: An Emphasis on Mammalian Spermatogenesis. HHS Author Manuscr. 2012;33(3):309-337. doi:10.2164/jandrol.111.014167

100. Lindqvist L, J P. Inhibitors of translation initiation as cancer therapeutics. Futur Med Chem. 2009;1(9):1709-1722. doi:10.4155/fmc.09.122

101. Murvin M, Banruo Y, Alec D, Christian F, Angie H. Methylation of Ded1 Affects Its Role in Translation. FASEB J. 2019. https://www.fasebj.org/doi/abs/10.1096/fasebj.2019.33.1_supplement.629.5. 
102. Bolinger C, Sharma A, Singh D, Yu L. RNA helicase A modulates translation of HIV1 and infectivity of progeny virions. Nucleic Acids Res. 2010;38(5):1686-1696. doi:10.1093/nar/gkp1075

103. Liwak U, Faye MD, Holcik M. Translation control in apoptosis. Exp Oncol. 2012;34(3):218-230. http://exp-oncology.com.ua/article/3645/translationcontrol-in-apoptosis.

104. Malina A, Mills JR, Pelletier J. Emerging Therapeutics Targeting mRNA Translation. Cold Spring Harb Perspect Biol. 2012;4(4):1-17. doi:10.1101/cshperspect.a012377.

105. Li JJ, Chew G-L, Biggin MD. Quantitative principles of cis-translational control by general mRNA sequence features in eukaryotes. Genome Biol. 2019;20(1):1-24. doi:10.1186/s13059-019-1761-9

106. Golshani A, Golomehova V, Mironova R, Ivanov IG, AbouHaidar MG. Does the epsilon sequence of phage T7 function as an initiator for the translation of CAT mRNA in Escherichia coli? Biochem Biophys Res Commun. 1997;236(2):253-256. doi:10.1006/bbrc.1997.6842

107. Kaloyanova D, Xu J, Ivanov IG, Abouhaidar MG. Gene expression evidence indicates that nucleotides 507-513 and 1434-1440 s rRNA are organized in close proximity in 1 on the Escherichia coli 30s ribosomal subunit. Eur J Biochem. 1997;14:10-14. doi:DOI: 10.1111/j.1432-1033.1997.00010.x

108. Golshani A, Krogan NJ, Xu J, et al. Escherichia coli mRNAs with strong 
Shine/Dalgarno sequences also contain $5^{\prime}$ end sequences complementary to domain \# 17 on the 16S ribosomal RNA. Biochem Biophys Res Commun. 2004;316(4):978-983. doi:10.1016/j.bbrc.2004.02.169

109. Golshani A, Kolev V, Abouhaidar MG, Ivanov IG. Epsilon as an initiator of translation of CAT mRNA in Escherichia coli. Biochem Biophys Res Commun. 2000;273(2):528-531. doi:10.1006/bbrc.2000.2958

110. Picard F, Milhem H, Loubière $P$, Laurent $B$, Cocaign-bousquet $M$, Girbal L. Bacterial translational regulations : high diversity between all mRNAs and major role in gene expression. BMC Genomics. 2012;13:528. doi:10.1186/1471-2164-13528

111. Bentley-DeSousa A, Holinier C, Moteshareie H, et al. A Screen for Candidate Targets of Lysine Polyphosphorylation Uncovers a Conserved Network Implicated in Ribosome Biogenesis. Cell Rep. 2018;22(13):3427-3439. doi:10.1016/j.celrep.2018.02.104

112. Gebauer F, Preiss T, Hentze MW. From Ci s -Regulatory Elements to Complex RNPs and Back. Cold Spring Harb Perspect Biol. 2012;4(7):1-14. doi:10.1101/cshperspect.a012245

113. Nandagopal N, Roux PP. Regulation of global and specific mRNA translation by the mTOR signaling pathway. Translation. 2015;3(1):e983402. doi:10.4161/21690731.2014.983402 
114. Roux PP, Topisirovic I. Signaling Pathways Involved in the Regulation of mRNA Translation. Mol Cell Biol. 2018;38(12):1-26. doi:10.1128/mcb.00070-18

115. Rojas-duran MF, Gilbert W V. Alternative transcription start site selection leads to large differences in translation activity in yeast. RNA. 2012;(Gilbert 2010):22992305. doi:10.1261/rna.035865.112.mRNAs

116. Cuperus JT, Groves B, Kuchina A, et al. Deep learning of the regulatory grammar of yeast 5 ' untranslated regions from 500 , 000 random sequences. Genome Res. 2017;27:2015-2024. doi:10.1101/gr.224964.117.7

117. Young SK, Wek RC. Upstream Open Reading Frames Differentially Regulate Genespecific Translation in the Integrated Stress Response. LBC. 2016;291(33):1692716935. doi:10.1074/jbc.R116.733899

118. Kulkarni SD, Zhou F, Sen ND, Zhang H, Hinnebusch AG, Lorsch JR. Temperaturedependent regulation of upstream open reading frame translation in S. cerevisiae. BMC Biol. 2019;17(1):1-27. doi:10.1186/s12915-019-0718-5

119. Chen HH, Yu HI, Yang MH, Tarn WY. DDX3 Activates CBC-elF3-Mediated translation of uORF-containing oncogenic mRNAs to promote metastasis in HNSCC. Cancer Res. 2018;78(16):4512-4523. doi:10.1158/0008-5472.CAN-180282

120. Thakor N, Holcik M. IRES-mediated translation of cellular messenger RNA operates in elF $2 \alpha$-independent manner during stress. Nucleic Acids Res. 
2012;40(2):541-552. doi:10.1093/nar/gkr701

121. Holcik M, Sonenberg N. TRANSLATIONAL CONTROL IN STRESS AND APOPTOSIS. Nat Rev Mol Cell Biol. 2005;6(April):318-327. doi:10.1038/nrm1618

122. Pooggin M, Ryabova L, He X, Futterer J, Hohn T. Mechanism of ribosome shunting in Rice tungro bacilliform pararetrovirus. RNA. 2006;12:841-850. doi:10.1261/rna.2285806.Xi

123. Martin F, Barends S, Jaeger S, Schaeffer L, Prongidi-fix L, Eriani G. Cap-Assisted Internal Initiation of Translation of Histone H4. Mol Cell. 2011;41(2):197-209. doi:10.1016/j.molcel.2010.12.019

124. Vivinus S, Baulande S, Zanten M Van, et al. An element within the $5^{\prime}$ untranslated region of human Hsp70 mRNA which acts as a general enhancer of mRNA translation. Eur J Biochem. 2001;1917:1908-1917. doi:10.1046/j.14321327.2001.02064.x

125. Du Z, Alekhina OM, Vassilenko KS, Simon AE. Concerted action of two $3^{\prime}$ capindependent translation enhancers increases the competitive strength of translated viral genomes. Nucleic Acids Res. 2017;45(16):9558-9572. doi:10.1093/nar/gkx643

126. Golshani A, Kolev V, Mironova R, Abouhaidar MG, Ivanov IG. Enhancing activity of $\varepsilon$ in Escherichia coli and Agrobacterium tumefaciens cells. Biochem Biophys Res Commun. 2000;269(2):508-512. doi:10.1006/bbrc.2000.2327 
127. Takahashi S, Furusawa H, Ueda T, Okahata Y. Translation enhancer improves the ribosome liberation from translation initiation. J Am Chem Soc.

2013;135(35):13096-13106. doi:10.1021/ja405967h

128. Couvillion MT, Soto IC, Shipkovenska G, Churchman LS. Synchronized mitochondrial and cytosolic translation programs. Nature. 2016;533(7604):499503. doi:10.1038/nature18015.Synchronized

129. Bonnefoy N, Bsat N, Fox TD. Mitochondrial Translation of Saccharomyces cerevisiae COX2 mRNA Is Controlled by the Nucleotide Sequence Specifying the Pre-Cox2p Leader Peptide. Mol Cell Biol. 2001;21(7):2359-2372. doi:10.1128/MCB.21.7.2359-2372.2001

130. Schneider A. Mitochondrial tRNA Import and Its Consequences for Mitochondrial Translation. Annu Rev Biochem. 2011;80:1033-1053. doi:10.1146/annurevbiochem-060109-092838

131. Firczuk H, Kannambath S, Pahle J, et al. An in vivo control map for the eukaryotic mRNA translation machinery. Mol Syst Biol. 2013;9(635):1-13. doi:10.1038/msb.2012.73

132. Sloan KE, Bohnsack MT. Unravelling the Mechanisms of RNA Helicase Regulation. Trends Biochem Sci. 2018;43(4):237-250. doi:10.1016/j.tibs.2018.02.001

133. Leeuwen W Van, Rabouille C. Cellular stress leads to the formation of membraneless stress assemblies in eukaryotic cells. Traffic. 2019;(May):623-638. 
doi:10.1111/tra.12669

134. Sen ND, Zhou F, Ingolia NT, Hinnebusch AG. Genome-wide analysis of translational efficiency reveals distinct but overlapping functions of yeast DEADbox RNA helicases Ded1 and elF4A. Genome Res. 2015;25:1196-1205. doi:10.1101/gr.191601.115.1196

135. Soto-rifo R, Rubilar PS, Limousin T, Breyne S De. DEAD-box protein DDX3 associates with elF4F to promote translation of selected mRNAs. EMBO J. 2012;31(18):3745-3756. doi:10.1038/emboj.2012.220

136. Pisareve V., Pisarev A., Komar A., Hellen CU., Pestova T. Translation initiation on mammalian mRNAs with structured 5'- UTRs requires DExH-box protein DHX29. Cell. 2008;135(7):1237-1250. doi:10.1016/j.cell.2008.10.037.Translation

137. Abaeva IS, Marintchev A, Pisareva VP, Hellen CUT, Pestova T V. Bypassing of stems versus linear base-by-base inspection of mammalian mRNAs during ribosomal scanning. EMBO J. 2011;30(1):115-129. doi:10.1038/emboj.2010.302

138. Outten C, Culotta V. Alternative start sites in the Saccharomyces cerevisiae GLR1 gene are responsible for mitochondrial and cytosolic isoforms of glutathione reductase. J Biol Chem. 2004;279(9)::7785-91.

doi:10.1074/jbc.M312421200.Alternative

139. Geissmann T, Marzi S, Romby P, Geissmann T, Marzi S, Romby P. The role of mRNA structure in translational control in bacteria. RNA Biol. 2009;6286:153-160. 
doi:10.4161/rna.6.2.8047

140. Lenox RH, Wang L. Molecular basis of lithium action : integration of lithiumresponsive signaling and gene expression networks. Mol Psychiatry. 2003;8:135144. doi:10.1038/sj.mp.4001306

141. Won E, Kim Y. An Oldie but Goodie : Lithium in the Treatment of Bipolar Disorder through Neuroprotective and Neurotrophic Mechanisms. Int J Mol Sci. 2017;18(12):2679. doi:10.3390/ijms18122679

142. Fornaro M, Berardis D De, Anastasia A, et al. The identification of biomarkers predicting acute and maintenance lithium treatment response in bipolar disorder : A plea for further research attention. Psychiatry Res. 2018;269:658672. doi:10.1016/j.psychres.2018.08.034

143. Ariyasinghe D, Perera SR. The role of lithium in the treatment of bipolar disorder. Sri Lanka J Psychiatry. 2018:28-30. doi:10.4038/sljpsyc.v9i2.8190

144. Yang D, Song L, Hu J, et al. Biochemical and Biophysical Research Communications Enhanced tolerance to $\mathrm{NaCl}$ and $\mathrm{LiCl}$ stresses by over-expressing Caragana korshinskii sodium / proton exchanger 1 ( CkNHX1) and the hydrophilic C terminus is required for the activity of $\mathrm{CkNHX1}$ in Atsos3. Biochem Biophys Res Commun. 2012;417(2):732-737. doi:10.1016/j.bbrc.2011.12.023

145. Sofola-adesakin O, Castillo-quan J., Rallis C, et al. Lithium suppresses A $\beta$ pathology by inhibiting translation in an adult Drosophila model of Alzheimer' $s$ 
disease. Front Aging Neurosci. 2014;6(July):1-10. doi:10.3389/fnagi.2014.00190

146. Castillo-Quan J., Li L, Kinghorn K., Hardy J, Bjedov I, Partridge L. Lithium Promotes Longevity through GSK3 / NRF2- Dependent Hormesis Lithium Promotes Longevity. Cell Rep. 2016;15:638-650. doi:10.1016/j.celrep.2016.03.041

147. Williams RS., Harwood A. Lithium therapy and signal transduction. Trends Pharmacol Sci. 2000;21(2):61-64. doi:10.1016/S0165-6147(99)01428-5

148. Montero-Lomelí M, Morais BLB, Figueiredo DL, Neto DCS, Martins JRP, Masuda CA. The initiation factor elF4A is involved in the response to lithium stress in Saccharomyces cerevisiae. J Biol Chem. 2002;277(24):21542-21548. doi:10.1074/jbc.M201977200

149. Bro C, Regenberg B, Lagniel G, Labarre J, Montero-Lomelí M, Nielsen J. Transcriptional, proteomic, and metabolic responses to lithium in galactosegrown yeast cells. J Biol Chem. 2003;278(34):32141-32149. doi:10.1074/jbc.M304478200

150. Masuda CA, Xavier MA, Mattos KA, Galina A, Montero-Lomeli M. Phosphoglucomutase Is an in Vivo Lithium Target in Yeast. Biol Chem. 2001;276:37794-37801. doi:10.1074/jbc.M101451200

151. Liu J., Zhang GC, Kong I., et al. A mutation in PGM2 causing inefficient Galactose metabolism in the Probiotic Yeast Saccharomyces boulardii. Appl enviromental Microbiol. 2018;84(10):1-12. doi:10.1128/AEM.02858-17 
152. Dichtl B, Stevens A, Tollervey D. Lithium toxicity in yeast is due to the inhibition of RNA processing enzymes. EMBO J. 1997;16(23):7184-7195. doi:10.1093/emboj/16.23.7184

153. Bergkessel M, Whitworth G., Guthrie C. Diverse environmental stresses elicit distinct responses at the level of pre-mRNA processing in yeast. RNA. 2011;17:1461-1478. doi:10.1261/rna.2754011

154. Winzeler EA, Shoemaker DD, Astromoff A, et al. Functional Characterization of the S . cerevisiae Genome by Gene Deletion and Parallel Analysis. Science (80- ). 1999;285(5429):901-907. doi:10.1126/science.285.5429.901

155. Gelperin DM, White MA, Wilkinson ML, et al. Biochemical and genetic analysis of the yeast proteome with a movable ORF collection. Genes Dev. 2005:2816-2826. doi:10.1101/gad.1362105.The

156. Karlsson-rosenthal C, Millar JBA. Cdc25 : mechanisms of checkpoint inhibition and recovery. Trends Cell Biol. 2006;16(6). doi:10.1016/j.tcb.2006.04.002

157. Altmann M, Müller P., Wittmer B, Ruchti F, Lanker S, Trachsel H. A Saccharomyces cerevisiae homologue of mammalian translation initiation factor $4 \mathrm{~B}$ contributes to RNA helicase activity. EMBO J. 1993;12(10):3997-4003. doi:10.1002/j.14602075.1993.tb06077.x

158. Stansfield I, Akhmaloka M, Tuite F. A mutant allele of the SUP45 (SAL4) gene of Saccharomyces cerevisiae shows temperature-dependent aUosuppressor and 
omnipotent suppressor phenotypes. Curr Genet. 1995;27:417-426.

doi:10.1007/bf00311210

159. Szymanski EP, Kerscher O. Budding Yeast Protein Extraction and Purification for the Study of Function, Interactions, and Post-translational Modifications. J Vis Exp. 2013;80(October):1-9. doi:10.3791/50921

160. Samanfar B, Tan LH, Shostak K, et al. A global investigation of gene deletion strains that affect premature stop codon bypass in yeast, Saccharomyces cerevisiae. Mol Biosyst. 2014;10(4):916-924. doi:10.1039/c3mb70501c

161. Lee Y, Kim Y. Progress in Neuro-Psychopharmacology \& Biological Psychiatry The impact of glycogen synthase kinase $3 \beta$ gene on psychotic mania in bipolar disorder patients. Prog Neuropsychopharmacol Biol Psychiatry. 2011;35(5):13031308. doi:10.1016/j.pnpbp.2011.04.006

162. Alamgir M, Eroukova V, Jessulat M, Xu J, Golshani A. Chemical-genetic profile analysis in yeast suggests that a previously uncharacterized open reading frame, YBR261C, affects protein synthesis. BMC Genomics. 2008;9:1-13. doi:10.1186/1471-2164-9-583

163. Nagalakshmi U, Wang Z, Waern K, et al. The Transcriptional Landscape of the Yeast Genome Defined by RNA Sequencing. Science (80- ). 2008;320(June):13441350. doi:1126/science. 1158441

164. Washietl S, Hofacker IL, Lukasser M, Hüttenhofer A, Stadler PF. Mapping of 
conserved RNA secondary structures predicts thousands of functional noncoding RNAs in the human genome. Nat Biotechnol. 2005;23(11):1383-1390. doi:10.1038/nbt1144

165. Toufighi K, Youn J, Ou J, et al. Quantitative analysis of fitness and genetic interactions in yeast on a genome scale. Nat Methods. 2011;7(12):1017-1024. doi:10.1038/nmeth.1534.Quantitative

166. Hendry JA, Tan G, Ou J, Boone C, Brown GW. Leveraging DNA Damage Response Signaling to Identify Yeast Genes Controlling Genome Stability. G3 Genes / Genomes / Genetics. 2015;5(May):997-1006. doi:10.1534/g3.115.016576

167. Ooi SL, Pan X, Peyser BD, et al. Global synthetic-lethality analysis and yeast functional profiling. Trends Genet. 2006;22(1):56-63. doi:10.1016/j.tig.2005.11.003

168. Douglas AC, Smith AM, Sharifpoor S, et al. Functional Analysis With a Barcoder Yeast Gene Overexpression System. G3 Genes/Genomes/Genetics. 2012;2(10):1279-1289. doi:10.1534/g3.112.003400

169. Li X, Ohmori T, Irie K, et al. Different Regulations of ROM2 and LRG1 Expression by Ccr4, Pop2, and Dhh1 in the Saccharomyces cerevisiae Cell Wall Integrity Pathway. mSphere. 2016;1(5). doi:10.1128/mSphere.00250-16

170. Castelli LM, Lui J, Campbell SG, et al. Glucose depletion inhibits translation initiation via elF4A loss and subsequent 48S preinitiation complex accumulation, 
while the pentose phosphate pathway is coordinately up-regulated. Mol Biol Cell. 2011;22(15):3379-3393. doi:10.1091/mbc.E11-02-0153

171. Blewett NH, Goldstrohm AC. A Eukaryotic Translation Initiation Factor 4E-Binding Protein Promotes mRNA Decapping and Is Required for PUF Repression. mcb. 2012;32(20):4181-4194. doi:10.1128/MCB.00483-12

172. Fischer N, Weis K. The DEAD box protein Dhh1 stimulates the decapping enzyme Dcp1. EMBO J. 2002;21(11):2788-2797. doi:10.1093/emboj/21.11.2788

173. Carroll JS, Munchel SE, Weis K. The DExD/H box ATPase Dhh1 functions in translational repression, mRNA decay, and processing body dynamics. cell Biol. 2011;194(4):527-537. doi:10.1083/jcb.201007151

174. Yan P, Xu D, Ji Y, et al. LiCl Pretreatment Ameliorates Adolescent Methamphetamine Exposure-Induced Long-Term Alterations in Behavior and Hippocampal Ultrastructure in Adulthood in Mice. Int J Neuropsychopharmacol. 2019;22(4):303-316. doi:10.1093/ijnp/pyz001

175. Chiu C-T, Chuang D-M. Molecular actions and therapeutic potential of lithium in preclinical and clinical studies of CNS disorders. Pharmacol Ther. 2011;128(2):281304. doi:10.1016/j.pharmthera.2010.07.006.Molecular

176. Hull M, Lee E, Lee T, Anand N, Lalone V. Lithium Chloride Induces TNF a in Mouse Macrophages Via. Cell Biochem. 2014;80(April 2013):71-80. doi:10.1002/jcb.24634 
177. Zhang J. Lithium chloride promotes proliferation of neural stem cells in vitro, possibly by triggering the Wnt signaling pathway. Taylor Fr Gr. 2019;23(1):32-41. doi:https://doi.org/10.1080/19768354.2018.1487334 Lithium

178. Mu F, Huang J, Xing $\mathrm{T}$, et al. The $\mathrm{Wnt} / \beta$-catenin/Lef1 pathway promotes cell proliferation at least in part through direct upregulation of miR-17-92 cluster. Front Genet. 2019;10(MAY):1-12. doi:10.3389/fgene.2019.00525

179. Hampel H, Ewers M, Bürger K, et al. Lithium trial in Alzheimer's disease: A randomized, single-blind, placebo-controlled, multicenter 10-week study. J Clin Psychiatry. 2009;70(6):922-931. doi:10.4088/JCP.08m04606

180. Karásková M, Gunis S, Herrmannová A, Wagner S, Munzarová V. Functional Characterization of the Role of the N-terminal Domain of the $\mathrm{c}$ / Nip1 Subunit of Eukaryotic Initiation Factor 3 (elF3) in AUG Recognition. JBC. 2012;287(34):2842028434. doi:10.1074/jbc.M112.386656

181. Pisareva VP, Pisarev A V. DHX29 and elF3 cooperate in ribosomal scanning on structured mRNAs during translation initiation. Rna. 2016;22(12):1859-1870. doi:10.1261/rna.057851.116

182. Cuchalova L, Kouba T, Herrmannova A, Danyi I, Chiu W -I., Valasek L. The RRM of elF3g is required for resumption of scanning of post-termination ribosomes for reinitiation on GCN4 and together with elF3i stimulates linear scanning. Mol Cell Biol. 2010;30(19):4671-4686. doi:10.1128/mcb.00430-10 
183. Valášek L, Phan L, Schoenfeld LW, Valášková V, Hinnebusch AG. Related elF3 subunits TIF32 and HCR1 interact with an RNA recognition motif in PRT1 required for elF3 integrity and ribosome binding. EMBO J. 2001;20(4):891-904. doi:10.1093/emboj/20.4.891

184. Blasco-moreno B, Campos-mata L De, Böttcher R, et al. The exonuclease Xrn1 activates transcription and translation of mRNAs encoding membrane proteins. Nat Commun. 2019;(2019):1-15. doi:10.1038/s41467-019-09199-6

185. Suzuki Y, Minami M, Suzuki M, et al. The Hsp90 Inhibitor Geldanamycin Abrogates Colocalization of elF4E and elF4E-Transporter into Stress Granules and Association of elF4E with elF4G. J Biol Chem. 2009;284(51):35597-35604. doi:10.1074/jbc.M109.036285

186. Coordes B. Ctk1 function is crucial for efficient translation initiation and interacts with the mRNP processing factor Npl3. PhD Thesis Univ Munich. 2011. https://edoc.ub.uni-muenchen.de/13203/1/Coordes_Britta.pdf.

187. Coordes B, Brünger KM, Burger K, Soufi B, Horenk J, Eick D. Ctk1 Function Is Necessary for Full Translation Initiation Activity in Saccharomyces cerevisiae. Am Soc Microbiol. 2015;14(1):86-95. doi:10.1128/EC.00106-14

188. Fox TD. Mitochondrial protein synthesis, import, and assembly. Yeastbook, cell Struct Traffick. 2012;192(December):1203-1234. doi:10.1534/genetics.112.141267 
189. Falkenberg M, Larsson N, Gustafsson C. DNA Replication and Transcription in Mammalian Mitochondria. Annu Rev. 2007;76:679-699.

https://doi.org/10.1146/annurev.biochem.76.060305.152028.

190. Herrmann JM, Woellhaf MW, Bonnefoy N. Control of protein synthesis in yeast mitochondria : The concept of translational activators. BBA - Mol Cell Res. 2013;1833(2):286-294. doi:10.1016/j.bbamcr.2012.03.007

191. Galluzzi L, Kepp O, Kroemer G. Mitochondrial regulation of cell death : a phylogenetically conserved control. Micorbial Cell. 2016;3(3):101-108. doi:10.15698/mic2016.03.483

192. Schapira AH V. Mitochondrial disease. Lancet. 2006:70-82. doi:https://doi.org/10.1016/S0140-6736(06)68970-8

193. Weinberg SE, Chandel NS. Targeting mitochondria metabolism for cancer therapy. Nat Chem Biol. 2015;11(1):9-15. doi:10.1038/nchembio.1712

194. Weinberg SE, Sena LA, Chandel NS. Mitochondria in the Regulation of Innate and Adaptive Immunity. Immunity. 2015;42(3):406-417. doi:10.1016/j.immuni.2015.02.002

195. Wang G, Shimada E, Zhang J, Hong JS, Smith GM, Teitell MA. Correcting human mitochondrial mutations with targeted RNA import. PNAS. 2012;109(13). doi:10.1073/pnas.1116792109

196. Brand RM, Wipf P, Durham A, Epperly MW, Greenberger JS, Jr LDF. Targeting 
Mitochondrial Oxidative Stress to Mitigate UV-Induced Skin Damage. Front Pharmacol. 2018;9(August):1-10. doi:10.3389/fphar.2018.00920

197. Osman C, Noriega TR, Okreglak V, Fung JC, Walter P. Integrity of the yeast mitochondrial genome, but not its distribution and inheritance, relies on mitochondrial fission and fusion. PNAS. 2015:947-956.

doi:10.1073/pnas.1501737112

198. Kren BT, Wong PY, Sarver A, Zhang X, Zeng Y, Steer CJ. microRNAs identified in highly purified liver-derived mitochondria may play a role in apoptosis. RNA Biol. 2009;6(1):65-72. doi:10.4161/rna.6.1.7534

199. Zhang H, Singh KK. Global Genetic Determinants of Mitochondrial DNA Copy Number. Plose one. 2014;9(8):1-12. doi:10.1371/journal.pone.0105242

200. Ott M, Herrmann JM. Co-translational membrane insertion of mitochondrially encoded proteins. BBA - Mol Cell Res. 2010;1803(6):767-775. doi:10.1016/j.bbamcr.2009.11.010

201. Gadir N, Haim-vilmovsky L, Kraut-cohen J, Gerst JE. Localization of mRNAs coding for mitochondrial proteins in the yeast Saccharomyces cerevisiae. RNA Biol. 2011:1551-1565. doi:10.1261/rna.2621111

202. Sylvestre J, Margeot A, Jacq C, Dujardin G. The Role of the 3' Untranslated Region in mRNA Sorting to the Vicinity of Mitochondria Is Conserved from Yeast to Human Cells. Mol Biol Cell. 2003;14(September):3848-3856. 
doi:10.1091/mbc.e03-02-0074

203. Laso MRV, Zhus D, Sagliocco F, Brown AJP, Mick F, Mccarthyl JEG. Inhibition of Translational Initiation in the Yeast Saccharomyces Position of Hairpin cereuisiae as a Function of the Stability and Structures in the mRNA Leader. Biol Chem. 1993;268(9). http://www.jbc.org/content/268/9/6453.abstract.

204. Dunstan HM, Green-willms NS, Fox TD. In Vivo analysis of Saccharomyces cerevisiae COX2 mRNA 5'-untraslated leader functions in Mitochondrial translation initiation and translational activation. Genet Soc Am. 1997;147:87100. https://www.ncbi.nlm.nih.gov/pmc/articles/PMC1208125/.

205. Green-willms NS, Butler CA, Dunstan HM, Fox TD. Pet111p , an Inner Membranebound Translational Activator That Limits Expression of the Saccharomyces cerevisiae Mitochondrial Gene COX2. Biol Chem. 2001;276(9):6392-6397. doi:10.1074/jbc.M009856200

206. Federico A, Cardaioli E, Pozzo P Da, Formichi P, Gallus GN, Radi E. Mitochondria, oxidative stress and neurodegeneration. J Neurol Sci. 2012;322(1-2):254-262. doi:10.1016/j.jns.2012.05.030

207. Alston CL, Rocha MC, Lax NZ, Turnbull DM, Taylor RW. The genetics and pathology of mitochondrial disease. Pathology. 2017;(November 2016):236-250. doi:10.1002/path.4809

208. Murat P, Marsico G, Herdy B, Ghanbarian A, Portella G, Balasubramanian S. RNA 
G-quadruplexes mark repressive upstream open reading frames in human mRNAs. bioRxiv. 2017;19(1):229. doi:10.1186/s13059-018-1602-2

209. Horvilleur E, Sbarrato T, Hill K, et al. A role for eukaryotic initiation factor 4B overexpression in the pathogenesis of diffuse large B-cell lymphoma. Leukemia. 2013;28(5):1092-1102. doi:10.1038/leu.2013.295

210. Kawai S, Hashimoto W, Murata K. Transformation of Saccharomyces cerevisiae and other fungi: methods and possible underlying mechanism. Bioeng Bugs. 2010;1(6):395-403. doi:10.4161/bbug.1.6.13257

211. Inoue $\mathrm{H}$, Nojima $\mathrm{H}$, Okayama $\mathrm{H}$. High efficiency transformation of Escherichia coli with plasmids. Gene. 1990;96:23-28. doi:10.1016/0378-1119(90)90336-P

212. Reid RJD, González-barrera S, Sunjevaric I, Alvaro D, Wagner M, Rothstein R. Selective Ploidy Ablation, a high throughput plasmid transfer protocol, identifies new genes affecting topoisomerase I - induced DNA damage. Genome Res. 2010. doi:10.1101/gr.109033.110

213. Serebriiskii IG, Golemis EA. Uses of lacZ to study gene function: Evaluation of $\beta$ galactosidase assays employed in the yeast two-hybrid system. Anal Biochem. 2000;285(1):1-15. doi:10.1006/abio.2000.4672

214. Bourgeois CF, Mortreux F, Auboeuf D. The multiple functions of RNA helicases as drivers and regulators of gene expression. Nat Rev Mol Cell Biol. 2016;17(7):426438. doi:10.1038/nrm.2016.50 
215. Rodríguez-Galán O, García-Gómez JJ, De la Cruz J. Yeast and human RNA helicases involved in ribosome biogenesis: Current status and perspectives. BBA gene Regul Mech. 2013;1829(8):775-790. doi:10.1016/j.bbagrm.2013.01.007

216. Rodríguez-Galán O, García-Gómez JJ, De la Cruz J. Yeast and human RNA helicases involved in ribosome biogenesis: Current status and perspectives. Biochim Biophys Acta - Gene Regul Mech. 2013;1829(8):775-790. doi:10.1016/j.bbagrm.2013.01.007

217. Leppek K, Das R, Barna M. Functional 5' UTR mRNA structures in eukaryotic translation regulation and how to find them. Nat Rev Mol Cell Biol. 2018;19(3):158-174. doi:10.1038/nrm.2017.103

218. Munteanu A, Mukherjee N, Ohler U. SSMART: sequence-structure motif identification for RNA-binding proteins. Bioinformatics. 2018;34(23):3990-3998. doi:10.1093/bioinformatics/bty404

219. Feracci M, Foot JN, Grellscheid SN, et al. Structural basis of RNA recognition and dimerization by the STAR proteins T-STAR and Sam68. Nat Commun. 2016;7:1-12. doi:10.1038/ncomms10355

220. Tuller T, Ruppin E, Kupiec M. Properties of untranslated regions of the $S$. cerevisiae genome. BMC Genomics. 2009;10(391). doi:10.1186/1471-2164-10-391 


\section{Appendix}

\subsection{Additional data for chapter 2 and 4}

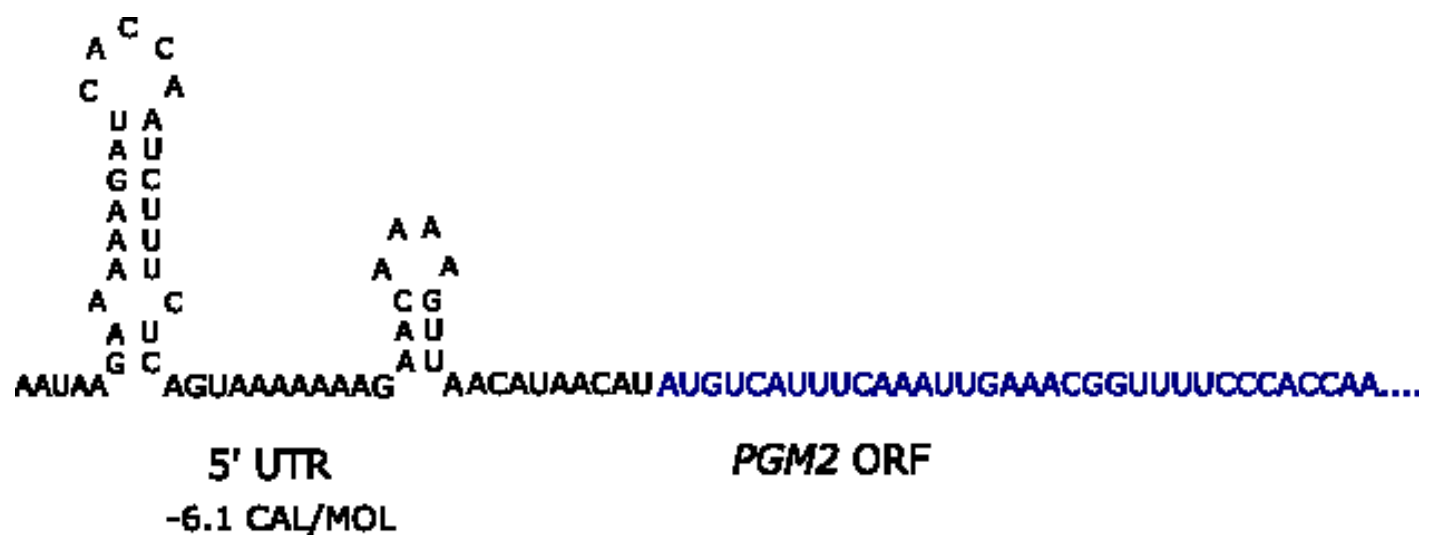

Figure 8.1. The secondary structure of PGM2 5'-UTR. Unlike most yeast ORFs, the 5'UTR of PGM2 is thought to be structured (Tuller et al., 2009).

\subsection{List of primers used for gene knock out in chapters 2 and 4. Blue and Red} color letters are showing complementary regions with NAT sequence in pAG25. Black letters are complementary regions with upstream and downstream of target genes for knock out. Confirmation primers (Conf) are designed for gene replacement confirmation with NAT resistance marker.

\begin{tabular}{|l|l|}
\hline Primer name & Sequence \\
\hline YTA6-Forward & $\begin{array}{l}\text { AGAGGTAAGACCCTGGGTGAAGAAAAGTCCGAGGAGGGAACACAAA } \\
\text { AAAGTCTAGGATACACATACGATTTAGGTGACAC }\end{array}$ \\
\hline YTA6-Reverse & $\begin{array}{l}\text { GTACATAGGTAATAAAATTAGATCAGAAGCAAGAAGTTGAAAGGGG } \\
\text { AAAATTCCTTCTCAATACGACTCACTATAGGGAG }\end{array}$ \\
\hline YTA6-Conf & AAGGCGGGGCCAAACGAGGCAATC \\
\hline
\end{tabular}




\begin{tabular}{|l|l|}
\hline YPR096C-Forward & $\begin{array}{l}\text { TTCCTTGTTCACGCCTAAAAAGTTAACTGTCAAATGGTTAACGTACATT } \\
\text { TATCTGTCTTCACATACGATTTAGGTGACAC }\end{array}$ \\
\hline YPR096C-Reverse & $\begin{array}{l}\text { GTTGAAACAGAGTAAATAATAGGGAGTGGATGCGAGTAACCTTGCG } \\
\text { ATGTCAAAAGAATAATACGACTCACTATAGGGAG }\end{array}$ \\
\hline YPR096C-Conf & GATAGGCTGTGGAGCTGTCGGATCGTTTGG \\
\hline NAM7-Forward & $\begin{array}{l}\text { AGGAAGGGCAGCAAGACCGAATATACTTTTTATATTACATCAATCATT } \\
\text { GTCATTATCAACACATACGATTTAGGTGACAC }\end{array}$ \\
\hline NAM7-Reverse & $\begin{array}{l}\text { TTGAGCCGTTTTGTACACAAGCCAAGTTTAACATTTTATTTTAACAGG } \\
\text { GTTCACCGAAATACGACTCACTATAGGGAG }\end{array}$ \\
\hline NAM7-Conf & TTGCCTGGAGGAAAGGAATCCCAGGAGA \\
\hline PUS2-Forward & $\begin{array}{l}\text { TTACTCCGACACCGTTAACAGCGGTAGTAAAGAGAAGGCCGAGTAAA } \\
\text { CATAAGGTCGTTCACATACGATTTAGGTGACAC }\end{array}$ \\
\hline PUS2-Reverse & $\begin{array}{l}\text { CAACTGGCGCTTGTCTAAGTTGAATAGCCAATAAGATGAGAGTATTG } \\
\text { CCCGCCCAATGTAATACGACTCACTATAGGGAG }\end{array}$ \\
\hline PUS2-Conf & GGGCCTCCCGCATAAGAACGCACT CC \\
\hline RPL27B-Forward & $\begin{array}{l}\text { TAGTGACTATAGGGCGTTTGGTGAAAAGAAAGAGTCGCTCAAAGAA } \\
\text { ATCAATATAACACATACGATTTAGGTGACAC }\end{array}$ \\
\hline RPL27B-Reverse & $\begin{array}{l}\text { AAACTGCATTATGAAAATAATAATAGTACACAGAAAAAAGTTAGGTTT } \\
\text { CGGAAAAAAGCAATACGACTCACTATAGGGAG }\end{array}$ \\
\hline RPL27B-Conf & CTGCCTAGATTGCGCGGAGGGTACGTTG \\
\hline NAT-Conf & TCCAGTGCCTCGATGGCCTCGGCG \\
\hline
\end{tabular}

8.3. 6-galactosidase quantification data for chapter 5. 6-galactosidase activity of candidate genes, selected from lift assay screening, was performed to confirm the reduction in the translation of 6 -galactosidase. The data was normalized to that of WT carrying either of the constructs p2hair, pB-cell, pRTN, and pTAR. 

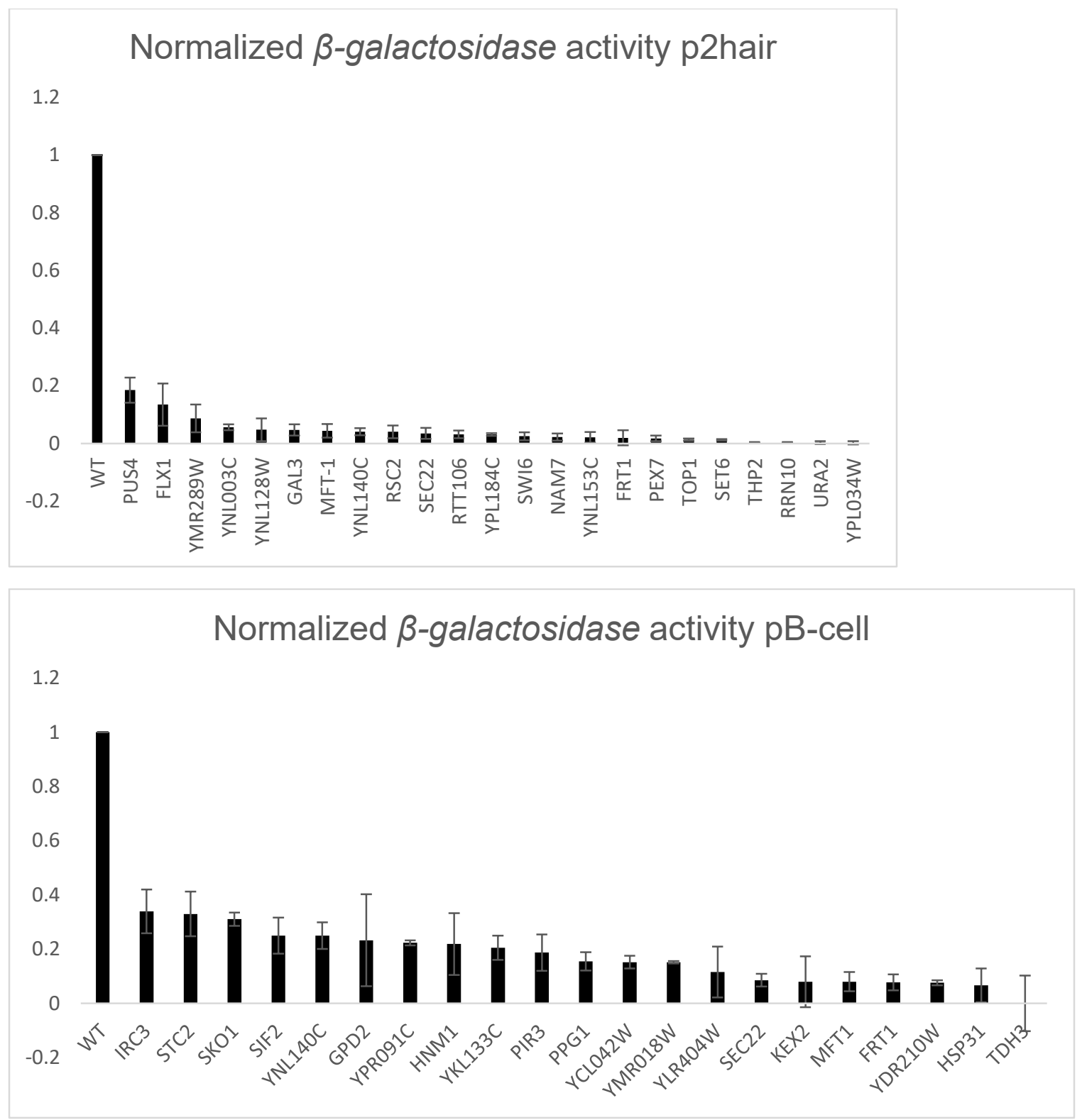

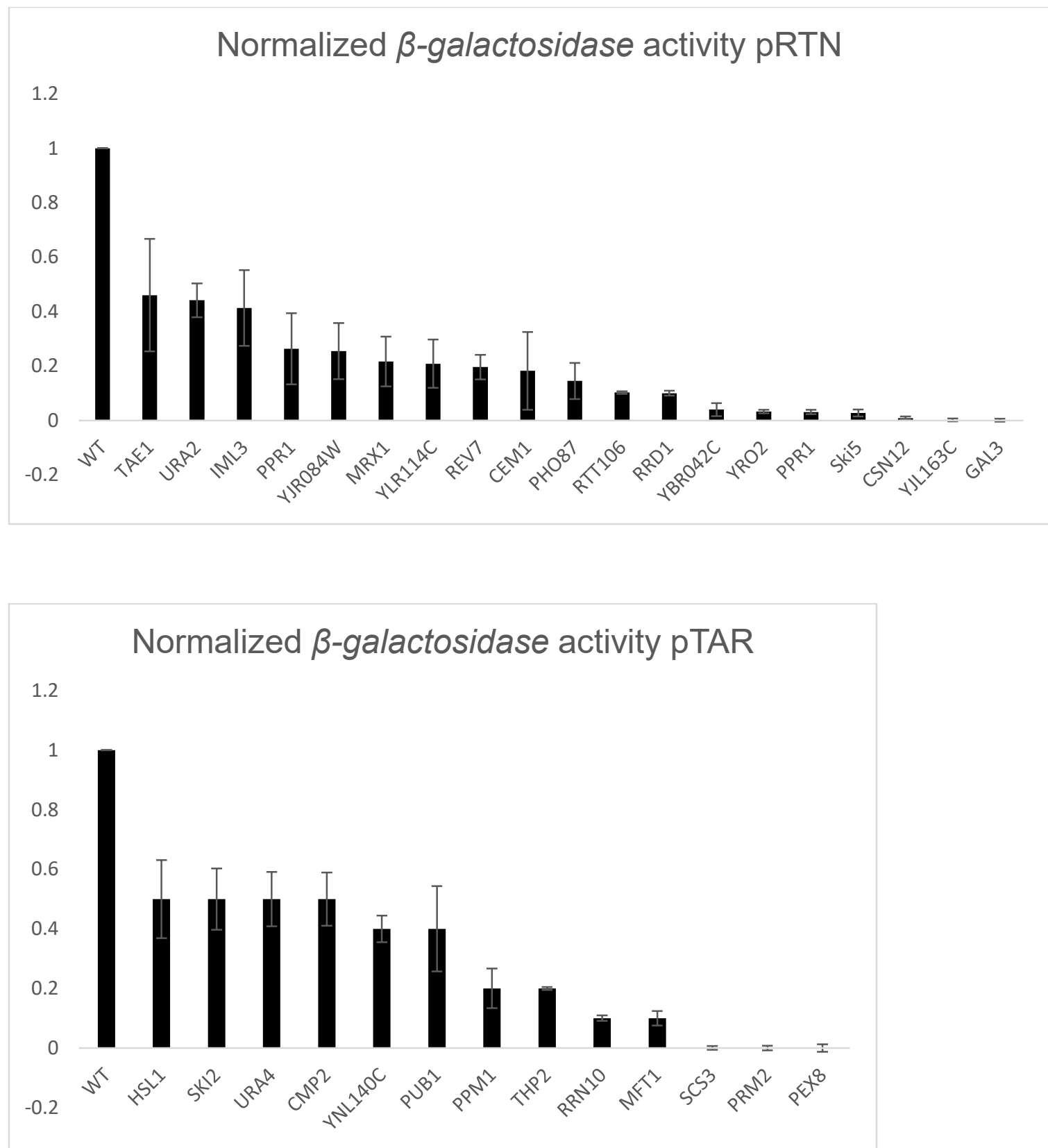


\subsection{List of candidate genes selected from 6-galactosidase assay for constructs pTAR, pRTN, pBcell, and p2hair. Function summary of each candidate gens is presented.}

\begin{tabular}{|c|c|}
\hline Ptar candidate genes & Function summary \\
\hline RRN10 & Regulation in transcription \\
\hline RPP1A & involved in phosphorylation of elF2 \\
\hline PEX7 & $\begin{array}{l}\text { peroxisomal receptor (function as transcription factors } \\
\text { regulating gene expression) }\end{array}$ \\
\hline PPM1 & $\begin{array}{l}\text { Carboxyl transferase important in complex formation of } \\
\text { regulatory subunits and required for methionine to inhibit } \\
\text { autophagy }\end{array}$ \\
\hline SCS3 & required for inositol prototrophy for regulation of normal ER \\
\hline THP2 and MFT1 & $\begin{array}{l}\text { involev in transcription elongation and RNA polymerase II, } \\
\text { affecting mRNA export }\end{array}$ \\
\hline MET18 & $\begin{array}{l}\text { component of cytosolic iron-sulfur protein assembly for } \\
\text { maturation of some proteins involved in methionine } \\
\text { biosynthesis, DNA replication and repair, transcription. }\end{array}$ \\
\hline HSL1 & protein kinase regulating morphogenesis checkpoint \\
\hline SK12 & $\begin{array}{l}\text { putative RNA helicase. Mediates 3'-5' RNA degradation in } \\
\text { exosome important in mRNA decay }\end{array}$ \\
\hline URA4 & biosynthesis of pyrimidines \\
\hline CMP2 & $\begin{array}{l}\text { regulated protein phosphatase involved in stress-response } \\
\text { transcription factors }\end{array}$ \\
\hline YMR226C & an oxireductaze enzyme, may interact with ribosome \\
\hline PUB1 & $\begin{array}{l}\text { poly (A) binding protein, required for stability of many mRNAs. } \\
\text { Involved in P-bodies. Translatoi regulation. Protien abundance } \\
\text { in response to DNA replication stress }\end{array}$ \\
\hline YNL140C & unknown \\
\hline YOR072W & unknown \\
\hline
\end{tabular}

\begin{tabular}{|c|l|}
\hline PRTN candidate genes & Function summary \\
\hline SWH1 & $\begin{array}{l}\text { oxysterol-binding proiten that interacts with ER and is regulated } \\
\text { by sterol bindings. }\end{array}$ \\
\hline RPL23A & ribosomal subunit \\
\hline CST26 & $\begin{array}{l}\text { Acetyltransferase. An enzyme reposible for incorporation of } \\
\text { stearic acid into phosphatidylinositol }\end{array}$ \\
\hline YRO2 & putative role in response to acid stress. \\
\hline IML3 and SLI15 & $\begin{array}{l}\text { both important in regulation of kinetochore-microtuble } \\
\text { activatoin and mitotic checkpoints }\end{array}$ \\
\hline TAE1 & methyltransferase, putative role in translatoin \\
\hline PHO87 & phosphate transporter \\
\hline GAL3 & transcriptional regulator \\
\hline
\end{tabular}




\begin{tabular}{|c|l|}
\hline SBH2 & involved in protein translocation into ER. \\
\hline CEM1 & a synthase required for mitochondrial respiration. \\
\hline MRX1 & $\begin{array}{l}\text { associates with mitochondrial ribosome and its mutation } \\
\text { results in a decrease in plasma membrane electron transport. }\end{array}$ \\
\hline SAP4 & protein required for function of the Sit4 protein phosphate. \\
\hline SMI1 & $\begin{array}{l}\text { protein involved in the regulation of cell wall synthesis in } \\
\text { coordination with cell cycle progression. }\end{array}$ \\
\hline YIL067C & unknown \\
\hline REV7 & $\begin{array}{l}\text { subunit of DNA polymerase zeta involved in translesion } \\
\text { synthesis in post-replication repair. Involved in double strand } \\
\text { break repair. It has domains that are found in proteins involved } \\
\text { in cell cycle control, meiosis and DNA reparl. }\end{array}$ \\
\hline RRD1 & $\begin{array}{l}\text { activator of the phosphotyrosyl phosphatase activity of PP2A. } \\
\text { Required for rapid reduction of Sgs1p in response to rapamycin. } \\
\text { Increases its abundance in DNA replicaton stress. }\end{array}$ \\
\hline GUT2 & $\begin{array}{l}\text { mitochondrial glycerol-3-phosphate dehydrogenase. Invovled in } \\
\text { glycerol metabolism and replicative cell aging. }\end{array}$ \\
\hline YJL45W & $\begin{array}{l}\text { succinate dehydrogenase isozyme involved in cellular } \\
\text { respiration, localizes to mitochondria }\end{array}$ \\
\hline SKI3 & $\begin{array}{l}\text { carbomylphsphate synthesase involved in biosynthesis of } \\
\text { pyrimidines. Because Ura2p is intergral in regulating synhtesis } \\
\text { of pyrimidine nucleotides and this process is important in } \\
\text { cancer cell metabolism. }\end{array}$ \\
\hline PRR1 & $\begin{array}{l}\text { unknown } \\
\text { putative role in transcription elongation and mRNA splicing. }\end{array}$ \\
\hline YJL163C & $\begin{array}{l}\text { transciptin factor regulate transcription of genes involved in } \\
\text { pyrimidine biosynthesis in response to pyrimidine starvation. }\end{array}$ \\
\hline YJR084W & $\begin{array}{l}\text { involved in exocytic transport from Goli. Abundance upon DNA } \\
\text { replication stress. }\end{array}$ \\
\hline & $\begin{array}{l}\text { Histone chaperone. Involved in regulation of chromatin } \\
\text { structure in both transcribed and silenced chromosmal regions. }\end{array}$ \\
\hline & $\begin{array}{l}\text { mediates 3'-5' RNA degradation in exosome important in mRNA } \\
\text { decay. Nonsense mediated decay and non stop mRNA decay } \\
\text { also mRNAs unadenylated and viral dsRNA. }\end{array}$ \\
\hline &
\end{tabular}

\begin{tabular}{|c|l|}
\hline pBCell candidate genes & Function summary \\
\hline SIF2 & $\begin{array}{l}\text { subunit of Set3C histone deacetylase complex, repressing early } \\
\text { sporulation genes }\end{array}$ \\
\hline YCL042W & unknown \\
\hline PTC1 & $\begin{array}{l}\text { phosphate involved in inactivation of MAPK activity involved in } \\
\text { cell wall intergrity. Pheomone dependent signal transduction } \\
\text { and tRNA splicing. }\end{array}$ \\
\hline YDR210W & possible plasma membrane related to stress response \\
\hline IRC3 & $\begin{array}{l}\text { DNA helicase, deletion cause double stranded breaks in } \mathrm{mt} \\
\text { DNA. }\end{array}$ \\
\hline
\end{tabular}




\begin{tabular}{|c|c|}
\hline HSP31 & $\begin{array}{l}\text { involved in oxidattive stress resistance (defficiency is involved } \\
\text { in Parkinson's disease and cancer) contain metal-binding stie. } \\
\text { Abundance increases in response to DNA replication and stress. }\end{array}$ \\
\hline LSB3 & $\begin{array}{l}\text { involved in actin filamen assembly. Abundance increases in } \\
\text { response to DNA replicatoin stress. }\end{array}$ \\
\hline HNM1 & $\begin{array}{l}\text { plasma membrane transporter active during hypersaline stress } \\
\text { localized to ER. }\end{array}$ \\
\hline PEX31 & $\begin{array}{l}\text { Peroxisomal integral membrane protien (a small organelle } \\
\text { containing enzyme catalases and oxidases). }\end{array}$ \\
\hline TDH3 & $\begin{array}{l}\text { Glyceraldehyde-3-phosphate dehydrogenase involved in } \\
\text { glycolysis, gluconeogenesisi, apoptosis and the matabolism of } \\
\text { reactive oxygen species. Binds to AU rich RNAs. }\end{array}$ \\
\hline RGT1 & $\begin{array}{l}\text { DNA-binding transcription factro, acts both as activator and } \\
\text { repressor; involved in regulation of glucose metabolism }\end{array}$ \\
\hline $\mathrm{RCl} 50$ & unknown \\
\hline PIR3 & $\begin{array}{l}\text { paralog of HSP150. required for cell wall stability. Coding } \\
\text { sequence contains length polymorphisms repeats. }\end{array}$ \\
\hline SEC22 & $\begin{array}{l}\text { SNARE protein a group of large protein complex with primary } \\
\text { role to mediate vesicle fusion to their target in membrane } \\
\text { bound compartments. Deletin cause defective in maturation of } \\
\text { cell wall bound proteins and increase sensitivity to cell wall } \\
\text { affecting chemicals. }\end{array}$ \\
\hline SEI1 & $\begin{array}{l}\text { protein of the endoplasmic reticulum involved in lipid droplet } \\
\text { biogenesis. Stabilizing these sites on ER and deletion causes } \\
\text { accumulation of phophatidic acid (PA) marker proteins. }\end{array}$ \\
\hline MFT1 & $\begin{array}{l}\text { involev in transcription elongation and RNA polymerase II, } \\
\text { affecting mRNA export }\end{array}$ \\
\hline PEX9 & $\begin{array}{l}\text { Putative signal receptor for peroximsoma matrix proteins; } \\
\text { involved in the import of protiens into the peroxisomal matrix. } \\
\text { Its conditionn specific receptro. }\end{array}$ \\
\hline FET3 & $\begin{array}{l}\text { integral membrane multicopper oxidase which is required for } \\
\text { high-affinity iron uptade and invovled in mediating resistance } \\
\text { to copper ion toxicity and abundance increases in response to } \\
\text { DNA replication stress. }\end{array}$ \\
\hline YNL140C & unknown \\
\hline SKO1 & $\begin{array}{l}\text { transcription factor involved in a complex that both activate } \\
\text { and repress transcription. Also involved in osmotic and } \\
\text { oxidative stress responses. Suppressor of kinase overexpression }\end{array}$ \\
\hline KEX2 & $\begin{array}{l}\text { Kexin a proprotein convertase ( these proteins remove block } \\
\text { chains on other protiens to activate them) it is calcium } \\
\text { dependent and activates proteins on ER (secretory pathway) }\end{array}$ \\
\hline PPG1 & $\begin{array}{l}\text { Putative protein phosphotase, regulates other protien } \\
\text { phosphatases. }\end{array}$ \\
\hline GPD2 & $\begin{array}{l}\text { glycerol-3-phosphate dehydrogenase involved in glycerol } \\
\text { metabolism and oxidation loicalized in cytoplasm and mt. it is } \\
\text { controlled by oxygen-independent signaling pathway required }\end{array}$ \\
\hline
\end{tabular}




\begin{tabular}{|c|l|}
\hline & $\begin{array}{l}\text { to regulate metabolism under anoxic conditions. Inactivated by } \\
\text { energy-stress responsice kinase SNF1. }\end{array}$ \\
\hline TGL5 & $\begin{array}{l}\text { triacylglycerol lipase and lysophosphaticid acid (LPA) } \\
\text { acyltransferase. Potential substrate of cyclin dependent kinase } \\
\text { (CDC28). }\end{array}$ \\
\hline WTM1 & $\begin{array}{l}\text { Transcription factor that regulates meiosis, chromatin silencing } \\
\text { inhibits ribonucleotide reductase by sequestering it in the } \\
\text { nucleus. }\end{array}$ \\
\hline FRT1 & $\begin{array}{l}\text { an ER membrane protein of unknown function. Substrate of the } \\
\text { phospharase calcineurin that promotes cell growth in stress } \\
\text { conditions possible via a role in posttranslational translocation. }\end{array}$ \\
\hline RPS6A & $\begin{array}{l}\text { ribosomal subunit involved in maturaion of the small subunit } \\
\text { rRNA and translatoin. Phosphorylated ina TORC1-dependent } \\
\text { manner. }\end{array}$ \\
\hline NVJ2 & $\begin{array}{l}\text { lipid binding protein in ER involved in nonvesicular transfer of } \\
\text { ceramides form ER to Glogi. May interact with ribosome. }\end{array}$ \\
\hline NCA2 & $\begin{array}{l}\text { protein that regulates expression of Fo-F1 ATP synthase } \\
\text { subunits in mt. }\end{array}$ \\
\hline
\end{tabular}

\begin{tabular}{|c|l|}
\hline p2hair candidate genes & Function summary \\
\hline ERV46 & $\begin{array}{l}\text { Golgi-vesicle-mediated transport; integral to Golgi and ER } \\
\text { membrane. }\end{array}$ \\
\hline RRN10 & Regulation in transcription \\
\hline GAL3 & $\begin{array}{l}\text { mt intermembrane space protein of unknown function, protein } \\
\text { abundance increases in response to DNA replication stress. }\end{array}$ \\
\hline MIX14 & $\begin{array}{l}\text { peroxisomal receptor (function as transcription factors } \\
\text { regulating gene expression) }\end{array}$ \\
\hline PEX7 & putative drug transporter \\
\hline YDR338C & $\begin{array}{l}\text { Proteasome regulatory component (degrading protiens), } \\
\text { involved in mRNA export. Human ortholog DSS1 a BRCA1 } \\
\text { binding protein implicated in cancer. Abundance increase in } \\
\text { response to DNA replication stress. Deletin exhibits sensitivity } \\
\text { o heat and UV }\end{array}$ \\
\hline SEM1 & $\begin{array}{l}\text { cytoplasmic Lsm1p is involved in mRNA decay and possibly } \\
\text { involved in processing tRNA, snoRNA and rRNA }\end{array}$ \\
\hline LSM6 & $\begin{array}{l}\text { GTPase activator involved in signal transduction, actin } \\
\text { cytoskeleton organization and cell wall organization, potenital } \\
\text { substrate of Cycleyn-dependent kinases (cdc28) }\end{array}$ \\
\hline SAC7 & $\begin{array}{l}\text { sequence-specific DNA binding transcription factor during heat } \\
\text { stress and the G1/S mitotic transition. Deletion is sensitive to } \\
\text { DNA damage, antifungals, alcohols, cold. }\end{array}$ \\
\hline SW14 & $\begin{array}{l}\text { mt tRNA pseudouridine synthase, mt tRNA modification but } \\
\text { mutation also affects pseudouridylatin of some nuclear } \\
\text { encoded mRNAs. }\end{array}$ \\
\hline & \\
\hline &
\end{tabular}




\begin{tabular}{|c|c|}
\hline THP2 & $\begin{array}{l}\text { involev in transcription elongation and RNA polymerase II, } \\
\text { affecting mRNA export }\end{array}$ \\
\hline URA2 & $\begin{array}{l}\text { carbomylphsphate synthesase involved in biosynthesis of } \\
\text { pyrimidines. Because Ura2p is intergral in regulating synhtesis } \\
\text { of pyrimidine nucleotides and this process is important in } \\
\text { cancer cell metabolism. }\end{array}$ \\
\hline SWI6 & $\begin{array}{l}\text { transcriptin factor involved in meiotic gene expression and } \\
\text { regulate transcription at the G1/S transitin. Cell wall stress } \\
\text { induce phosphorylatoin of Mpk1p and that regulates Swi6p } \\
\text { localizatoi, required for unfolded protein response. }\end{array}$ \\
\hline SEC22 & $\begin{array}{l}\text { SNARE protein a group of large protein complex with primary } \\
\text { role to mediate vesicle fusion to their target in membrane } \\
\text { bound compartments. Deletin cause defective in maturation of } \\
\text { cell wall bound proteins and increase sensitivity to cell wall } \\
\text { affecting chemicals. }\end{array}$ \\
\hline $\mathrm{RSC2}$ & $\begin{array}{l}\text { subunit of RSC chromatin remodelong complex, and invovled in } \\
\text { transcripton regulatoin for mainraining chromosome } \\
\text { transmissino fidelity as well as DNA repair via UV damage } \\
\text { excisino, homologous recombination and nonhomologous end } \\
\text { joining. }\end{array}$ \\
\hline MFT1 & $\begin{array}{l}\text { involev in transcription elongation and RNA polymerase II, } \\
\text { affecting mRNA export }\end{array}$ \\
\hline NAM7 & $\begin{array}{l}\text { ATP-dependent RNA helcase involved in nonsense mediated } \\
\text { mRNA decay required for efficient translation termination at } \\
\text { nonsense codons and targeting NMD substrates to P-bodies. } \\
\text { Binds to Rps } 26 \text { in ribosome. And forms cytoplasmic foci upon } \\
\text { DNA relicatoin stress. }\end{array}$ \\
\hline HEF3 & $\begin{array}{l}\text { Translational elongation factor (EF3). Stimulates EF1 binding of } \\
\text { aminoacyl-tRNA by the ribosome. }\end{array}$ \\
\hline TEP1 & $\begin{array}{l}\text { inositol lipid phosphatase activity and homology of human } \\
\text { suppressor gene PTEN/MMAC1/TEP1 }\end{array}$ \\
\hline YNL140C & unkown \\
\hline RTT106 & $\begin{array}{l}\text { Histone chaperone. Involved in regulation of chromatin } \\
\text { structure in both transcribed and silenced chromosmal regions. }\end{array}$ \\
\hline CSE2 & $\begin{array}{l}\text { subunit of core mediator that acts as RNA polymerase II } \\
\text { coactivator required for regulatoin of transcripton by RNA } \\
\text { polymerase. Found in cytoplasm under hypoxia. In mutation } \\
\text { cell is sensitive to chemicals including caffeine, rapamaycin, } \\
\text { hygromycin B. }\end{array}$ \\
\hline TOP1 & $\begin{array}{l}\text { DNA topoisomerase the unwinds DNA, chromatin organizition, } \\
\text { DNA replication and transcription and in replication checkpoint } \\
\text { signalng. Target of antitumor drug camptothecin. Drug } \\
\text { increases the half-life enzyme DNA complex which results in } \\
\text { increase of double stranded DNA breaks during DNA } \\
\text { replicatoin. }\end{array}$ \\
\hline
\end{tabular}




\begin{tabular}{|c|l|}
\hline CIN5 & $\begin{array}{l}\text { transcription factor, mediates pleiotropic drug resistance and } \\
\text { salt tolerance, activated during oxidative stress. Overexpression } \\
\text { elevates resistance to mitomycin C and cisplatin }\end{array}$ \\
\hline FRT1 & $\begin{array}{l}\text { an ER membrane protein of unknown function. Substrate of the } \\
\text { phospharase calcineurin that promotes cell growth in stress } \\
\text { conditions possible via a role in posttranslational translocation. }\end{array}$ \\
\hline SKS1 & $\begin{array}{l}\text { putative serine/threonine protein kinase, suppressor kinase } \\
\text { SNF3 (plasma membrane). Invovled in adaptaion to low } \\
\text { concentratio of glucose }\end{array}$ \\
\hline YPL034W & unkown \\
\hline SET6 & SET domain of unknown function \\
\hline & $\begin{array}{l}\text { RNA-binding protein that may be involved in translatoinal } \\
\text { regulation; binds to specific categories of mRNAs, including } \\
\text { those that contain uORFs and IRES. Genetically interacts with } \\
\text { chromatin remodeling and splicing factors and mRNA } \\
\text { maturation. }\end{array}$ \\
\hline
\end{tabular}




\subsection{Selected manuscripts}

1. Burnside, D., Schoenrock, A., Moteshareie, H., Hooshyar M., Basra, P., Hajikarimlou, M., Dick, K., Barnes, B., Kazmirchuk, T., Jessulat, M., Pitre, S., Samanfar, B., Babu, M., Green., J., Wong, A., Dehne, F., Biggar, K and Golshani, A. (2019). In Silico Engineering of Synthetic Binding Proteins from Random Amino Acid Sequences in Silico Engineering of Synthetic Binding Proteins from Random Amino Acid Sequences. ISCIENCE, 11, 375-387. https://doi.org/10.1016/i.isci.2018.11.038.

2. Moteshareie, H., Hajikarimlou, M., Indrayanti, A. M., Burnside, D., Paula, A., Letti, C., Omidi, K., Kazmirchuk, T., Puchacz, N., Zare, N., Takallou, S., Naing., T., Hernandez, R.B., Willmore, W.G., Babu, M., McKay, B., Samanfar, B., Holcik, M and Golshani, A. (2018). Heavy metal sensitivities of gene deletion strains for ITT1 and RPS1A connect their activities to the expression of URE2, a key gene involved in metal detoxification in yeast. Plose One. 1-18. https://doi.org/10.1371/journal.pone.0198704.

3. Omidi, K., Jessulat, M., Hooshyar, M., Burnside, D., Schoenrock, A., Kazmirchuk, T., Hajikarimlou, M., Daniel, M., Moteshareiri, H., Bhojoo, U., Sanders, M., Ramotar, D., Dehne, F., Samanfar, B., Babu, M and Golshani, A. (2018). Uncharacterized ORF HUR1 in $\mathrm{fl}$ uences the e ffi ciency of non-homologous end-joining repair in Saccharomyces cerevisiae. Gene. 128-136. https://doi.org/10.1016/j.gene.2017.10.003. 
4. Kazmirchuk, T., Dick, K., Burnside, D., Barnes, B., Moteshareie, H., Hajikarimlou, M., Omidi, K., Ahmed., Duale., Low, A., Letti, C., Hooshyar, M., Schoenrock, A., Pitre, S., Babu, M., Cassol., E., Samanfar, B., Wong A., Dehne, F., Green J.R and Golshani A. (2017). Designing Anti-Zika Virus Peptides Derived from Predicted Human-Zika Virus Protein-Protein Interactions. Computational biology and chemistry. 71, 180-187. https://doi.org/10.1016/i.compbiolchem.2017.10.011

5. Samanfar, B., Shostak, K., Moteshareie, H., Hajikarimlou, M., Shaikho, S., Omidi, K., Hooshyar, M., Burnside, D., Marquez, I.G., Kazmirchuk, T., Naign, T., Ludovico, P., YorkIyon, A., Szereszewski, K., Leung, C., Jin, J.Y., Megarbance, R., Smith, M.L., Babu, M., Holcik, M and Golshani, A. (2017). The sensitivity of the yeast, Saccharomyces cerevisiae, to acetic acid is influenced by DOM34 and RPL36A. PeerJ, 2017(11). https://doi.org/10.7717/peeri.4037.

6. Shaikho, S., Dobson, C. C., Naing, T., Samanfar, B., Hajikarimloo, M., Golshani, A., \& Holcik, M. (2016). Elevated levels of ribosomal proteins eL36 and eL42 control expression of Hsp90 in rhabdomyosarcoma. Translation, 4(2), 1-12. https://doi.org/10.1080/21690731.2016.1244395. 
7. Barnes, B., Hajikarimlou, M., Schoenrock, A., Burnside, D., Cassol, E., Wong, A., Dehne, F., Golshani, A and Green, J.R. (2016.). Predicting Novel Protein-Protein Interactions Between the HIV-1 Virus and Homo Sapiens. 2016 IEEE EMBS International Student Conference $\quad$ (ISC), $\quad 1$ 1-4. https://doi.org/10.1109/EMBSISC.2016.7508598. 